\title{
MULTIPARAMETER SEMIGROUPS AND ATTRACTORS OF REACTION-DIFFUSION EQUATIONS IN $\mathbb{R}^{n}$
}

\author{
S. V. ZELIK
}

\begin{abstract}
The space-time dynamics generated by a system of reaction-diffusion equations in $\mathbb{R}^{n}$ on its global attractor are studied in this paper. To describe these dynamics the extended $(n+1)$-parameter semigroup generated by the solution operator of the system and the $n$-parameter group of spatial translations is introduced and their dynamic properties are studied. In particular, several new dynamic characteristics of the action of this semigroup on the attractor are constructed, generalizing the notions of fractal dimension and topological entropy, and relations between them are studied. Moreover, under certain natural conditions a description of the dynamics is obtained in terms of homeomorphic embeddings of multidimensional Bernoulli schemes with infinitely many symbols.
\end{abstract}

\section{Contents}

Introduction

1. Analytic properties of solutions of reaction-diffusion equations in $\mathbb{R}^{n}$

2. The global attractor of a nonlinear reaction-diffusion equation and its $\varepsilon$-entropy

3. The space-time topological entropy of the attractor

4. Generalized topological directional entropies

5 . The injectivity of the solution operator and the temporal evolution of spacechaotic structures

6. Strongly unstable manifolds of nonhyperbolic equilibria

7. Strongly unstable manifolds of reaction-diffusion equations and lower bounds for the $\varepsilon$-entropy of the attractor

8. Kotel'nikov's formula and space chaos in reaction-diffusion equations

9. Construction of the auxiliary spatial dynamical system

10. Space-time chaos in reaction-diffusion equations in $\mathbb{R}^{n}$

11. Formally gradient systems of reaction-diffusion equations and their topological entropy

\section{INTRODUCTION}

We study the space-time dynamics generated by the following quasilinear system of reaction-diffusion equations in $\mathbb{R}^{n}$ :

$$
\partial_{t} u=a \Delta_{x} u-\left(\vec{L}, \nabla_{x}\right) u-\lambda_{0} u-f(u),\left.\quad u\right|_{t=0}=u_{0}, \quad t \geq 0, \quad x \in \mathbb{R}^{n} .
$$

Here $u=\left(u_{1}(t, x), \ldots, u_{k}(t, x)\right)$ is an unknown vector function, $\Delta_{x}$ is the Laplace operator in the variables $x \in \mathbb{R}^{n}, a>0$ and $\lambda_{0}>0$ are given positive numbers,

2000 Mathematics Subject Classification. Primary 35B40, 37B40, 37L05.

Key words and phrases. Reaction-diffusion equations, multiparameter semigroups, topological entropy, space-time chaos.

This research was carried out with the partial support of the INTAS grant no. 00-899 and the CRDF grant no. 2343. 
$f(u)=\left(f_{1}\left(u_{1}, \ldots, u_{k}\right), \ldots, f_{k}\left(u_{1}, \ldots, u_{k}\right)\right)$ is a given nonlinear interaction function, and $\left(\vec{L}, \nabla_{x}\right) u$ is a transport term with the following form:

$$
\left(\vec{L}, \nabla_{x}\right) u:=\sum_{i=1}^{n} L_{i} \partial_{x_{i}} u
$$

where $\vec{L}:=\left(L_{1}, \ldots, L_{n}\right)$ is a given vector. We also assume that the nonlinearity $f(u)$ satisfies the following standard conditions, which guarantee that the dynamical system generated by $(0.1)$ is dissipative:

$$
\begin{aligned}
& \text { 1. } f \in C^{2}\left(\mathbb{R}^{k}, \mathbb{R}^{k}\right), \\
& \text { 2. } \quad f(v) . v \geq-C,
\end{aligned}
$$$$
\text { 3. } f^{\prime}(v) \geq-K \quad \forall v \in \mathbb{R}^{k} \text {, }
$$

where $C>0$ and $K>0$ are some fixed constants. (Here and in what follows, the symbol $u . v$ denotes the standard scalar product in $\mathbb{R}^{k}$ and the condition $f^{\prime}(v) \geq-K$ means that $f^{\prime}(v) \theta . \theta \geq-K|\theta|^{2}$ for any $\theta \in \mathbb{R}^{k}$.)

The notion of a global attractor plays a central role in the study of the dynamic properties of dissipative systems generated by the evolution equations of mathematical physics (see [1, 18, 28, 39] and the references therein). Indeed, when $\Omega \subset \mathbb{R}^{n}$ is a bounded domain, most of these equations have a global attractor with finite Hausdorff and fractal dimension. Thus, the notion of a global attractor means we can reduce our investigation of the original dynamical system in infinite-dimensional phase space, such as $\Phi=L^{2}(\Omega)$, to the study of a reduced system on the attractor $\mathcal{A}$, which is in some sense, finite dimensional. Therefore, although the original phase space is infinite dimensional, the dynamic properties of these dissipative systems turn out to be close to the properties of dynamical systems generated by ordinary differential equations and can be studied using the standard ideas and methods of the classical, finite-dimensional theory of dynamical systems (see [26, 36, 39] and the references therein).

The situation changes fundamentally when we move to the study of dissipative systems generated by the equations of mathematical physics in unbounded domains. In this case, as is well known (see, for example, [3, 14, 23, 24]), the attractor $\mathcal{A}$ usually has infinite fractal dimension. (See also [10] 14, 23] for certain special cases of dissipative systems in unbounded domains, which nonetheless have a finite-dimensional attractor.) Thus, in contrast to the case of a bounded domain $\Omega$, the dynamics generated by dissipative systems in unbounded domains are essentially infinite dimensional and so the classical "finite-dimensional" methods turn out not to be useful for studying them. Moreover, the existence of "unbounded" spatial directions means that the solutions have a very complicated spatial structure and so-called space chaos (see [11, 12, 43]). Space-time chaos also arises as a result of the interactions between spatial and temporal modes. (See, for example, 16, 35. where space-time chaos in model discrete dynamical systems on lattices is investigated.)

In this paper we will present a systematic study of the infinite-dimensional dynamics that emerge on attractors of dissipative systems in unbounded domains, taking as our model the system of equations (0.1). Our method is based on the following simple observation: since (0.1) is spatially homogeneous, its attractor $\mathcal{A}$ is invariant under the group $\left\{T_{h}, h \in \mathbb{R}^{n}\right\}$ of spatial translations:

$$
T_{h}: \mathcal{A} \rightarrow \mathcal{A}, \quad T_{h} \mathcal{A}=\mathcal{A}, \quad\left(T_{h} u_{0}\right)(x):=u_{0}(x+h), \quad h \in \mathbb{R}^{n} .
$$

Moreover, this group commutes with the evolutionary semigroup $\left\{S_{t}, t \geq 0\right\}$ generated by (0.1) on the attractor. Thus, the extended $(n+1)$-parameter semigroup

$$
\mathbb{S}_{(t, h)}:=S_{t} \circ T_{h}, \quad \mathbb{S}_{(t, h)}: \mathcal{A} \rightarrow \mathcal{A}, \quad t \geq 0, \quad h \in \mathbb{R}^{n},
$$


acts on the attractor $\mathcal{A}$ of (0.1). We will be interpreting the multiparameter semigroup $(0.5)$ as a dynamical system with multidimensional time acting on the attractor $\mathcal{A}$; we shall describe the space-time dynamics generated by equation (0.1) in terms of dynamic characteristics of this system. In particular, we will show that, in spite of a seemingly fundamental difference between the space and time directions in the dynamical system (0.5) ( $h \in \mathbb{R}^{n}$ and $t \in \mathbb{R}_{+}$, respectively), these directions are, in a sense, equivalent and can be studied within the framework of a single theory.

The paper has the following structure. In $\S 1$ we recall the standard analytic properties of solutions of equation (0.1), discussing various a priori estimates, the global existence of solutions, their uniqueness, smoothness, and so on.

In $\S 2$ we show that the semigroup $\left\{S_{t}, t \geq 0\right\}$ generated by equation (0.1) has a global locally compact attractor $\mathcal{A}$ in the phase space $\Phi_{b}:=L^{\infty}\left(\mathbb{R}^{n}\right)$, which is bounded in $\Phi_{b}$ and compact in $\Phi_{\text {loc }}:=L_{\text {loc }}^{\infty}\left(\mathbb{R}^{n}\right)$. In addition, we obtain several upper bounds for the Kolmogorov entropy of this attractor, which are of fundamental significance in what follows.

We have devoted $\S 3$ to the study of the topological entropy $h_{\text {top }}\left(\mathcal{A}, \mathbb{S}_{(t, h)}\right)$ of the action of the multiparameter semigroup (0.5) on the attractor $\mathcal{A}$. In particular, we obtain several equivalent formulae for calculating it. Using these we prove that

$$
h_{\mathrm{top}}\left(\mathcal{A}, \mathbb{S}_{(t, h)}\right) \leq C<\infty
$$

This result is a natural generalization to an unbounded domain, of the classical result that the topological entropy of the action of the evolutionary semigroup $S_{t}$ on the attractor $\mathcal{A}$ of $(0.1)$ in a bounded domain $\Omega$ is finite. Recently, in [33] examples of equations of the form $(0.1)$ for which this quantity is strictly positive were constructed.

In $\S 4$ we study the action on the attractor $\mathcal{A}$ of the subsemigroups $\mathbb{S}_{(t, h)}^{V_{k}}$ of the extended semigroup (0.5), that correspond to various $k$-dimensional hyperplanes $V_{k}$ in space-time $\mathbb{R}_{t} \times \mathbb{R}_{x}^{n}$ :

$$
\mathbb{S}_{(t, h)}^{V_{k}}:=\left\{\mathbb{S}_{(t, h)}, t \geq 0,(t, h) \in V_{k}\right\}, \quad \mathbb{S}_{(t, h)}^{V_{k}} \mathcal{A}=\mathcal{A}
$$

For example, choosing the one-dimensional hyperplane $V_{1}=\mathbb{R}_{t}$ corresponds to purely temporal dynamics $\left\{S_{t}, t \geq 0\right\}$; choosing $V_{n}=\mathbb{R}_{x}^{n}$, to purely spatial "dynamics" $\left\{T_{h}, h \in\right.$ $\left.\mathbb{R}^{n}\right\}$. An intermediate choice of the hyperplane $V_{k}$ describes the interaction of spatial and temporal modes of the system under consideration (generated, for example, by travelling waves). Note that, by contrast with the semigroup (0.5), the actions of the semigroups $(0.7)$ as a rule have infinite topological entropy: $h_{\text {top }}\left(\mathcal{A}, \mathbb{S}_{(t, h)}^{V_{k}}\right)=\infty$ if $k<n+1$ (see $\S 8$ and $\S 10$ ). Therefore, we introduce the notion of generalized topological entropies $\widehat{h}_{\text {top }}^{n+1-k}\left(\mathcal{A}, \mathbb{S}_{(t, h)}^{V_{k}}\right)$ of the action of $k$-parameter semigroups of the form (0.7) on the attractor $\mathcal{A}$ and prove that they are finite:

$$
\widehat{h}_{\text {top }}^{n+1-k}\left(\mathcal{A}, \mathbb{S}_{(t, h)}^{V_{k}}\right) \leq C<\infty .
$$

In addition, we obtain certain relations between the entropies corresponding to different hyperplanes $V_{k}$. These are a natural generalization to the multidimensional case of the classical inequality between the topological entropy and the fractal dimension.

In $\S 5$ we prove that the evolutionary operator $S_{t}$ is invertible on the attractor $\mathcal{A}$ and verify that the inverse operator $S_{-t}$ satisfies a Hölder condition (with index $\alpha$ arbitrarily close to 1 ). This result is used later to prove that quantitative complexity characteristics of the spatial structure of the initial condition $u_{0} \in \mathcal{A}$ are preserved under temporal evolution. 
In $\S 6$ we state and prove an abstract theorem on a strongly unstable manifold of a smooth mapping in a neighborhood of a nonhyperbolic equilibrium. This is of fundamental importance in obtaining lower bounds for the generalized entropies $\widehat{h}_{\text {top }}^{n+1-k}\left(\mathcal{A}, \mathbb{S}_{(t, h)}^{V_{k}}\right)$ and for describing the space-time dynamics (0.5) in terms of Bernoulli schemes.

In $\S 7$ we apply the abstract theorem in $\S 6$ to construct a strongly unstable manifold of a spatially homogeneous equilibrium of (0.1). Using this manifold we prove the existence of a homeomorphic embedding $\widetilde{\mathbb{V}}$ of the unit ball $\mathcal{B}(\sigma)$ in the classical space $\mathbb{B}_{\sigma}\left(\mathbb{R}^{n}\right)$ (the set of functions $u_{0} \in L^{\infty}\left(\mathbb{R}^{n}\right)$ whose Fourier transforms have support contained in the cube $\left.[-\sigma, \sigma]^{n}\right)$ into the attractor $\mathcal{A}$ commuting with the group of spatial translations:

$$
\widetilde{\mathbb{V}}:\left(\mathcal{B}(\sigma), T_{h}\right) \rightarrow\left(\mathcal{A}, T_{h}\right) .
$$

Thus, the group of spatial translations acting on the ball $\mathcal{B}(\sigma)$ can be interpreted as a universal model dynamical system for describing the spatial dynamics $\left(\mathcal{A}, T_{h}\right)$. In particular, it follows from $(0.9)$ that the generalized entropy $\widehat{h}_{\text {top }}^{1}\left(\mathcal{A}, T_{h}\right)$ corresponding to the spatial hyperplane $V_{n}=\mathbb{R}_{x}^{n}$ is strictly positive.

In $\S 8$, using a certain generalization of the classical Kotel'nikov-Cartwright formula for functions in $\mathbb{B}_{\sigma}$, we construct a homeomorphic embedding of the multidimensional Bernoulli scheme $\mathcal{M}_{n}:=[-1,1]^{\mathbb{Z}^{n}}$ with infinitely many symbols $\omega \in[-1,1]$ into the model dynamical system $\left(\mathcal{B}(\sigma), T_{h}\right)$ and therefore, due to the embedding (0.9), also into the spatial dynamics $\left(\mathcal{A}, T_{h}\right)$ on the attractor.

The main purpose of $\S 9$ and $\S 10$ is to obtain similar results for hyperplanes $V_{n}$ containing temporal directions, for example, $V_{n}=\operatorname{span}\left\{e_{t}, e_{x_{2}}, \ldots, e_{x_{n}}\right\}$. To do this, we assume that the vector $\vec{L}$ has the form $L e_{x_{1}}$ and consider the following auxiliary parabolic boundary-value problem in the domain $\Omega_{x_{1}}:=\left\{(t, x) \in \mathbb{R}^{n+1}, x_{1}>0\right\}$ :

$$
\partial_{t} u=a \Delta_{x} u-L \partial_{x_{1}} u-\lambda_{0} u-f(u),\left.\quad u\right|_{x_{1}=0}=u^{0},
$$

where the variable $x_{1}$ is interpreted as "time", and the variables $\left(t, x_{2}, \ldots, x_{n}\right)$, as space variables.

In particular, in $\S 9$ we prove that (0.10) does define a dissipative dynamical system $\mathcal{S}_{x_{1}}$ in the phase space $\Psi_{b}:=L^{\infty}\left(\mathbb{R}^{n}\right)$ if $L>0$ is sufficiently large. The attractors of equations (0.1) and (0.10), in a sense, coincide (see $\S 9$ ). Thus the description of the "spatial" dynamics generated by (0.10) gives a description of the space-time dynamics corresponding to the hyperplane $V_{n}$ generated by the original equation (0.1). In $\S 10$ we construct a strongly unstable manifold for the problem (0.10), in a similar way to $\S 7$, and we obtain a homeomorphic embedding

$$
\tau:\left(\mathcal{B}(\sigma), T_{h}\right) \rightarrow\left(\mathcal{A}, \mathbb{S}_{(t, h)}^{V_{n}}\right), \quad V_{n}=\operatorname{span}\left\{e_{t}, e_{x_{2}}, \ldots, e_{x_{n}}\right\}
$$

Moreover, (0.11) and the results of $\S 8$ yield a description of the space-time dynamics corresponding to the hyperplane $V_{n}$ based on the embedding of the Bernoulli scheme $\mathcal{M}_{n}:=$ $[-1,1]^{\mathbb{Z}^{n}}$. In particular, from this it follows that the generalized entropy $\widehat{h}_{\mathrm{top}}^{n}\left(\mathcal{A}, S_{t}\right)$ corresponding to the temporal "hyperplane" $V_{1}=\mathbb{R}_{t}$ is strictly positive:

$$
\widehat{h}_{\text {top }}^{n}\left(\mathcal{A}, S_{t}\right) \geq C>0 \quad \text { and therefore } \quad h_{\text {top }}\left(\mathcal{A}, S_{t}\right)=\infty .
$$

Finally, in $\S 11$ we prove that, in the case of a gradient nonlinearity $f(u)=\nabla_{u} F(u)$, the topological entropy of the multiparameter semigroup $(0.5)$ is equal to zero:

$$
h_{\text {top }}\left(\mathcal{A}, \mathbb{S}_{(t, h)}\right)=0 \text {. }
$$

This fact is a natural generalization of the classical result that the topological entropy of gradient systems of ordinary differential equations is equal to zero (see, for example, [6]).

In conclusion it is worth mentioning that the main results of the paper are apparently valid for a substantially broader class of dissipative equations than (0.1). For example, 
most of the results stated above remain valid in the general case of a nonscalar diffusion matrix $a$; see [43, 44]. Moreover, partial analogues to them were obtained in [4, 21, 42] for dissipative wave equations, and in [8], for elliptic equations in cylindrical domains.

\section{Analytic properties of SOlutions OF REACTION-DIFFUSION EQUATIONS IN $\mathbb{R}^{n}$}

In this section we show that (0.1) generates a dissipative semigroup in phase space $\Phi_{b}:=L^{\infty}\left(\mathbb{R}^{n}\right)$ and study its analytic properties. Since results of this type are fairly standard (see, for example, [5] or [23]), we just sketch the proofs below, leaving the details to the reader. We begin with the following theorem, which gives a dissipative estimate for solutions of $(0.1)$.

Theorem 1.1. Suppose that equation (0.1) satisfies the conditions stated in the Introduction. Then for any $u_{0} \in \Phi_{b}$ there exists a unique solution $u(t), t \geq 0$, of (0.1), and

$$
\|u(t)\|_{L^{\infty}\left(\mathbb{R}^{n}\right)} \leq C\left(\left\|u_{0}\right\|_{L^{\infty}\left(\mathbb{R}^{n}\right)} e^{-\alpha t}+1\right),
$$

where $C$ and $\alpha$ are some positive constants.

Proof. The derivation of (1.1) is based on the maximum principle for solutions of parabolic equations in $\mathbb{R}^{n}$. For if we let $w(t)=w(t, x):=|u(t)|^{2}$, by $(0.3)$, item 2 this function satisfies the inequality

$$
\begin{aligned}
\partial_{t} w-a \Delta_{x} w-\left(\vec{L}, \nabla_{x}\right) w+2 \lambda_{0} w & =-2 f(u(t)) \cdot u(t)-2 \nabla_{x} u(t) \cdot \nabla_{x} u(t) \leq 2 C, \\
\left.w\right|_{t=0} & =\left|u_{0}\right|^{2} .
\end{aligned}
$$

Applying the classical maximum principle (see, for example, [5]) to (1.2) we obtain

$$
|u(t, x)|^{2} \leq\left\|u_{0}\right\|_{L^{\infty}\left(\mathbb{R}^{n}\right)}^{2} e^{-2 \lambda_{0} t}+\frac{C}{\lambda_{0}},
$$

which proves the dissipative estimate (1.1). The existence and uniqueness of the solution $u(t)$ can be derived in the usual way from the a priori estimate (1.1) (see, for example, [5]). This proves Theorem 1.1.

Corollary 1.1. Suppose that the hypotheses of Theorem 1.1 hold. Then the solution $u(t)$ constructed in Theorem 1.1 satisfies

$$
\|u(t)\|_{C_{b}^{4-\delta}\left(\mathbb{R}^{n}\right)}+\left\|\partial_{t} u\right\|_{C_{b}\left([t, t+1] \times \mathbb{R}^{n}\right)} \leq Q_{\delta}\left(\left\|u_{0}\right\|_{L^{\infty}(\Omega)}\right) e^{-\alpha t}+C_{\delta}, \quad t \geq 1,
$$

for $t \geq 1$ and for an arbitrary $\delta>0$, where $\alpha$ and $C_{\delta}$ are some positive constants and $Q_{\delta}(z)$ is some monotone function depending on the form of the nonlinear function $f$ and the constant $\delta$.

Estimate (1.3) is a standard consequence of (1.1) and the classical interior estimates for solutions of quasilinear parabolic equations of the form (0.1) (see, for example, [5]). We only note that the explicit form of the smoothness index $4-\delta$ in (1.3) is determined by the assumption that the nonlinear function $f$ is $C^{2}$-smooth (see (0.3): $f \in C^{2}\left(\mathbb{R}^{k}, \mathbb{R}^{k}\right)$ ).

It follows from Theorem 1.1 that (0.1) defines a semigroup $\left\{S_{t} \geq 0\right\}$ in $\Phi_{b}$ by the following standard formula:

$$
S_{t}: \Phi_{b} \rightarrow \Phi_{b}, \quad S_{t} u_{0}:=u(t),
$$

where $u(t)$ is a solution of $(0.1)$ satisfying the initial conditions $u(0)=u_{0} \in \Phi_{b}$. Note also that equation (0.1) is spatially homogeneous. Consequently, the group $\left\{T_{h}, h \in \mathbb{R}^{n}\right\}$ of translations along spatial directions commutes with the evolutionary semigroup (1.4):

$$
T_{h} \circ S_{t}=S_{t} \circ T_{h}, \quad t \in \mathbb{R}_{+}, \quad h \in \mathbb{R}^{n}, \quad\left(T_{h} u_{0}\right)(x):=u_{0}(x+h) .
$$


Thus, (0.1) generates the action of the extended $(n+1)$-parameter semigroup $\left\{\mathbb{S}_{(t, h)}, t \in\right.$ $\left.\mathbb{R}_{+}, h \in \mathbb{R}^{n}\right\}$ in phase space $\Phi_{b}$ by the following obvious formula:

$$
\mathbb{S}_{(t, h)}:=S_{t} \circ T_{h}, \quad t \in \mathbb{R}_{+}, \quad h \in \mathbb{R}^{n}, \quad \mathbb{S}_{(t, h)}: \Phi_{b} \rightarrow \Phi_{b} .
$$

This multiparameter semigroup plays a key role in our study of the space-time structure of solutions of equation (0.1) in $\S \S 3-11$.

As the next step we prove that the evolutionary semigroup (1.4) is Lipschitzian with respect to the initial conditions $u_{0} \in \Phi_{b}$.

Theorem 1.2. Suppose that the hypotheses of Theorem 1.1 hold. Then for any two solutions $u(t)$ and $u_{1}(t)$ of equation (0.1) the following estimate holds:

$$
\left|u(t, x)-u_{1}(t, x)\right| \leq C e^{K t} \sup _{y \in \mathbb{R}^{n}}\left\{e^{-\alpha|x-y|}\left|u(0, y)-u_{1}(0, y)\right|\right\},
$$

where the constant $K$ is the same as in in (0.3), item 3, C is some positive constant, and $\alpha>0$ is a sufficiently small positive number.

Proof. We set $v(t):=u(t)-u_{1}(t)$. This function satisfies the equation

$$
\partial_{t} v-a \Delta_{x} v+\left(\vec{L}, \nabla_{x}\right) v+\lambda_{0} v=-l(t) v,\left.\quad v\right|_{t=0}=u(0)-u_{1}(0),
$$

where

$$
l(t):=\int_{0}^{1} f^{\prime}\left(s u(t)+(1-s) u_{1}(t)\right) d s .
$$

By $(0.3)$, item 3 the matrix $l(t)=l(t, x)$ satisfies the condition $l(t) \geq-K$. Hence the function $w(t)=w(t, x):=|v(t, x)|^{2}$ satisfies the differential inequality

$$
\partial_{t} w-a \Delta_{x} w+\left(\vec{L}, \nabla_{x}\right) w+2 \lambda_{0} w-2 K w \leq 0,\left.\quad w\right|_{t=0}=|v(0)|^{2} .
$$

Thus, by the maximum principle it is sufficient to prove an estimate of the form (1.7) for solutions of the linear equation

$$
\partial_{t} \theta-a \Delta_{x} \theta+\left(\vec{L}, \nabla_{x}\right) \theta+2 \lambda_{0} \theta-2 K \theta=0,\left.\quad \theta\right|_{t=0}=|v(0)|^{2} .
$$

To do this we introduce the weight function

$$
\phi_{\alpha, x_{0}}(x):=e^{-\alpha \sqrt{1+\left|x-x_{0}\right|^{2}}},
$$

where $\alpha$ is a sufficiently small positive number and $x_{0}$ is an arbitrary point in $\mathbb{R}^{n}$. Consider the function $\theta_{x_{0}}(t)=\theta_{x_{0}}(t, x):=\phi_{\alpha, x_{0}}(x) \theta(t, x)$, which clearly satisfies the equation

$$
\partial_{t} \theta_{x_{0}}-a \Delta_{x} \theta_{x_{0}}+\left(\vec{L}, \nabla_{x}\right) \theta_{x_{0}}+2\left(\lambda_{0}-K\right) \theta_{x_{0}}=L_{1}(x) \theta_{x_{0}}+L_{2}(x) \nabla_{x} \theta_{x_{0}},
$$

where

$$
\begin{aligned}
L_{1}(x) z & :=\left[2 a\left(\phi_{\alpha, x_{0}}^{-1}\left|\nabla_{x} \phi_{\alpha, x_{0}}\right|\right)^{2}-\phi_{\alpha, x_{0}}^{-1}\left(a \Delta_{x} \phi_{\alpha, x_{0}}-\left(\vec{L}, \nabla_{x}\right) \phi_{\alpha, x_{0}}\right)\right] z, \\
L_{2}(x) \nabla_{x} z & :=-2 a \phi_{\alpha, x_{0}}^{-1} \nabla_{x} \phi_{\alpha, x_{0}} . \nabla_{x} z .
\end{aligned}
$$

Now observe that the weight functions (1.10) satisfy

$$
\begin{aligned}
\left|\nabla_{x} \phi_{\alpha, x_{0}}(x)\right| & \leq \alpha \phi_{\alpha, x_{0}}(x), \\
\left|\Delta_{x} \phi_{\alpha, x_{0}}(x)\right| & \leq n\left(\alpha+\alpha^{2}\right) \phi_{\alpha, x_{0}}(x), \\
x & \in \mathbb{R}^{n} .
\end{aligned}
$$

Hence,

$$
\left|L_{1}(x) z\right| \leq C\left(\alpha+\alpha^{2}\right)|z|, \quad\left|L_{2}(x) \nabla_{x} z\right| \leq C\left(\alpha+\alpha^{2}\right)\left|\nabla_{x} z\right|,
$$


where $C$ is some constant independent of $\alpha, x$, and $x_{0}$. It follows from these estimates that if $C\left(\alpha+\alpha^{2}\right) \leq 2 \lambda_{0}$, the function $\widehat{\theta}(t):=\left\|\theta_{\alpha, x_{0}}(0)\right\|_{L^{\infty}\left(\mathbb{R}^{n}\right)} e^{2 K t}$ satisfies the inequality

$$
\partial_{t} \widehat{\theta}-a \Delta_{x} \widehat{\theta}+\left(\vec{L}, \nabla_{x}\right) \widehat{\theta}+\left(\lambda_{0}-2 K\right) \widehat{\theta}-L_{1}(x) \widehat{\theta}-L_{2}(x) \nabla_{x} \widehat{\theta} \geq 0,\left.\quad \widehat{\theta}\right|_{t=0} \geq\left.\theta_{x_{0}}\right|_{t=0} .
$$

Thus, according to the maximum principle

$$
\left|v\left(t, x_{0}\right)\right|^{2} \leq \theta\left(t, x_{0}\right)=\theta_{\alpha, x_{0}}\left(t, x_{0}\right) \leq \widehat{\theta}(t)=e^{2 K t}\left\|\phi_{\alpha / 2, x_{0}} v(0)\right\|_{L^{\infty}\left(\mathbb{R}^{n}\right)}^{2} .
$$

Since $x_{0} \in \mathbb{R}^{n}$ is arbitrary, (1.7) follows from (1.15). Theorem 1.2 is proved.

Corollary 1.2. Suppose that the hypotheses of Theorem 1.2 hold. Then for any two solutions $u(t)$ and $u_{1}(t)$ and for any $t \geq 1$ the following estimate holds:

$$
\begin{gathered}
\left|\nabla_{x} u(t, x)-\nabla_{x} u_{1}(t, x)\right| \leq C e^{K t} \sup _{y \in \mathbb{R}^{n}}\left\{e^{-\alpha|x-y|}\left|u(0, y)-u_{1}(0, y)\right|\right\}, \\
x \in \mathbb{R}^{n},
\end{gathered}
$$

where the constant $C$ depends on $\|u(0)\|_{\Phi_{b}}$ and $\left\|u_{1}(0)\right\|_{\Phi_{b}}$ but is independent of $t$ and $x$, while $\alpha>0$ is the same as in Theorem 1.2.

Just as for (1.3), (1.16) is a standard consequence of (1.7) and the classical interior estimate, but this time it is applied to the linear equation (1.8) (see [5, 23]).

Our next aim is to verify the fact that the semigroup (1.4) is Fréchet differentiable in the space $\Phi_{b}$. For that, as usual, we have to consider the following variational equation, which is the linearization of (0.1) along some (arbitrary) solution $u(t)=S_{t} u_{0}$ :

$$
\partial_{t} v=a \Delta_{x} v-\left(\vec{L}, \nabla_{x}\right) v-\lambda_{0} v-f^{\prime}(u(t)) v,\left.\quad v\right|_{t=0}=v_{0} .
$$

Proposition 1.1. Equation (1.17) has a unique solution $v(t)$, which satisfies

$$
|v(t, x)| \leq e^{K t} \sup _{y \in \mathbb{R}^{n}}\left\{e^{-\alpha|x-y|}\left|v_{0}(y)\right|\right\},
$$

where the constants $\alpha$ and $K$ are the same as in Theorem 1.2.

We omit the proof of (1.18) as it is word-for-word the same as the proof of Theorem 1.2. The existence of the solution $v(t)$ is a standard consequence of this estimate (just as for equation (0.1); see [5]).

We are now ready to prove that the semigroup (1.4) is differentiable.

Theorem 1.3. Suppose that the hypotheses of Theorem 1.1 hold. Let $u(t)$ and $u_{1}(t)$ be two arbitrary solutions of (0.1) and let $v(t)$ be the solution of (1.17) satisfying the initial condition $v(0)=u(0)-u_{1}(0)$. Then

$$
\begin{aligned}
& \left|u(t, x)-u_{1}(t, x)-v(t, x)\right| \\
& \quad \leq C e^{2 K t}|| u_{1}(0)-u_{2}(0) \|_{L^{\infty}\left(\mathbb{R}^{n}\right)} \sup _{y \in \mathbb{R}^{n}}\left\{e^{-\alpha|x-y|}\left|u(0, y)-u_{1}(0, y)\right|\right\},
\end{aligned}
$$

where the constants $K$ and $\alpha$ are the same as in Theorem 1.2 and the constant $C$ depends on the $L^{\infty}$-norms of the initial conditions $u(0)$ and $u_{1}(0)$ but is independent of $t \in \mathbb{R}_{+}$ and $x \in \mathbb{R}^{n}$.

Proof. We introduce the function $w(t):=u(t)-u_{1}(t)-v(t)$, which obviously satisfies the equation

$$
\partial_{t} w-a \Delta_{x} w+\left(\vec{L}, \nabla_{x}\right) w+\lambda_{0} w+f^{\prime}(u(t)) w=h_{u, u_{1}}(t),
$$

where

$$
h_{u, u_{1}}(t):=\int_{0}^{1}\left[f^{\prime}\left(s u(t)+(1-s) u_{1}(t)\right)-f^{\prime}(u(t))\right] d s \cdot v(t) .
$$


Since $f \in C^{2}\left(\mathbb{R}^{k}, \mathbb{R}^{k}\right)$ and by Theorem 1.1 the $L^{\infty}$-norms of the solutions $u(t)$ and $u_{1}(t)$ are bounded, the right-hand side of (1.20) admits the estimate

$$
\left|h_{u, u_{1}}(t, x)\right| \leq C\left|u(t, x)-u_{1}(t, x)\right| \cdot|v(t, x)|,
$$

where $C$ depends on the $L^{\infty}$-norms of the initial conditions but is independent of $t$ and $x$. By substituting (1.7) and (1.18) into the right-hand side of (1.21) we obtain

$$
\begin{aligned}
& \left|h_{u, u_{1}}(t, x)\right| \\
& \quad \leq C_{1} e^{2 K t}\left\|u(0)-u_{1}(0)\right\|_{L^{\infty}\left(\mathbb{R}^{n}\right)} \sup _{y \in \mathbb{R}^{n}}\left\{e^{-\alpha|x-y|}\left|u(0, y)-u_{1}(0, y)\right|\right\} .
\end{aligned}
$$

The required estimate, (1.19) can be derived from (1.20) and (1.22) in the same way as in the proof of Theorem 1.2. This completes the proof of Theorem 1.3.

Corollary 1.3. Suppose the hypotheses of Theorem 1.1 hold. Then the semigroup (1.4) belongs to the class $C^{1,1}\left(\Phi_{b}, \Phi_{b}\right)$ and its Fréchet derivative $D_{u_{0}} S_{t}\left(u_{0}\right)$ is calculated by the formula $D_{u_{0}} S_{t}\left(u_{0}\right) \xi:=v_{\xi}(t)$, where $\xi \in \Phi_{b}$ is an arbitrary vector and $v_{\xi}(t)$ is the solution of equation (1.17) that satisfies the initial condition $v_{\xi}(0)=\xi$. In addition, the following estimate holds:

$$
\left\|D_{u_{0}} S_{t}(u)-D_{u_{0}} S_{t}\left(u_{1}\right)\right\|_{\mathcal{L}\left(\Phi_{b}, \Phi_{b}\right)} \leq C_{2} e^{2 K t}\left\|u_{0}-u_{1}\right\|_{\Phi_{b}}, \quad u_{0}, u_{1} \in \Phi_{b},
$$

where the constant $C_{2}$ depends on the $L^{\infty}$-norms of the vectors $u_{0}$ and $u_{1}$.

Indeed, both the differentiability of the semigroup (1.4) and the estimate (1.23) follow immediately from (1.19).

To conclude this section we introduce a certain natural class of weight functions in $\mathbb{R}^{n}$ and the corresponding class of weighted spaces, which will be used repeatedly in what follows.

Definition 1.1. A function $\phi \in C_{\mathrm{loc}}\left(\mathbb{R}^{n}\right)$ is called a weight function with exponential growth rate $\mu>0$ if the following inequalities hold:

$$
\phi(x)>0 \quad \forall x \in \mathbb{R}^{n} \quad \text { and } \quad \phi(x+y) \leq C_{\phi} e^{\mu|x|} \phi(y) \quad \forall x, y \in \mathbb{R}^{n},
$$

where $C_{\phi}>0$ is some constant independent of $x$ and $y$. Let $\phi$ be an arbitrary weight function with exponential growth. The weighted space $\Phi_{\phi}:=L_{\phi}^{\infty}\left(\mathbb{R}^{n}\right)$ is defined by

$$
L_{\phi}^{\infty}\left(\mathbb{R}^{n}\right):=\left\{u \in L_{\mathrm{loc}}^{\infty}\left(\mathbb{R}^{n}\right),\|u\|_{L_{\phi}^{\infty}\left(\mathbb{R}^{n}\right)}:=\sup _{x \in \mathbb{R}^{n}}\{\phi(x)|u(x)|\}<\infty\right\} .
$$

The simplest properties of the weight functions defined above are collected in the following proposition.

Proposition 1.2. $\quad$ 1. Let $\phi$ be a weight function with exponential growth. Then, along with (1.24), the following estimate holds:

$$
\phi(x+y) \geq C_{\phi}^{-1} e^{-\mu|x|} \phi(y) \quad \forall x, y \in \mathbb{R}^{n} .
$$

2. The function $\varphi(x):=e^{-\alpha|x|}, \alpha \in \mathbb{R}$, is a weight function with exponential growth rate $|\alpha|$ (with constant $C_{\varphi}=1$ ) and the function $\psi_{\alpha}(x):=(1+|x|)^{\alpha}, \alpha \in \mathbb{R}$, is a weight function with exponential growth rate $\mu$ for any $\mu>0$.

3. If $\phi$ and $\phi_{1}$ are two weight functions with exponential growth, then the functions $\phi+\phi_{1}, \phi \cdot \phi_{1}$, and $\phi^{\alpha}(\alpha \in \mathbb{R})$ are also weight functions with exponential growth.

4. If $\phi$ is a weight function with exponential growth, then the functions $T_{h} \phi$ ( $h \in$ $\left.\mathbb{R}^{n}\right)$ and

$$
\phi_{V}(x):=\sup _{y \in V} \phi(x+y),
$$

where $V$ is an arbitrary bounded subset of $\mathbb{R}^{n}$, are weight functions with exponential growth and satisfy (1.24) with the same constants $\mu$ and $C_{\phi}$ as the original function $\phi$. 
5. For any function $u \in L_{\phi}^{\infty}\left(\mathbb{R}^{n}\right)$, where $\phi$ is an arbitrary weight function with exponential growth rate $\mu$, the following estimates hold:

$$
\|u\|_{L_{\phi}^{\infty}\left(\mathbb{R}^{n}\right)} \leq \sup _{y \in \mathbb{R}^{n}}\left\{\phi(y) \sup _{x \in \mathbb{R}^{n}}\left\{e^{-\mu|x-y|}|u(x)|\right\}\right\} \leq C_{\phi}\|u\|_{L_{\phi}^{\infty}\left(\mathbb{R}^{n}\right)} .
$$

Proof. The first four parts of Proposition 1.2 are obvious (see also [23, 43]). It remains to prove (1.28). The left-hand inequality in (1.28) is also obvious, since

$$
u(y) \leq \sup _{x \in \mathbb{R}^{n}}\left\{e^{-\mu|x-y|}|u(x)|\right\} .
$$

We now prove the right-hand inequality. Using (1.24) we obtain

$$
\begin{aligned}
\sup _{y \in \mathbb{R}^{n}} & \left\{\phi(y) \sup _{x \in \mathbb{R}^{n}}\left\{e^{-\mu|x-y|}|u(x)|\right\}\right\} \\
& \leq C_{\phi} \sup _{x, y \in \mathbb{R}^{n}}\left\{\phi(x) e^{\mu|x-y|} e^{-\mu|x-y|}|u(x)|\right\}=C_{\phi}\|u\|_{L_{\phi}^{\infty}\left(\mathbb{R}^{n}\right)} .
\end{aligned}
$$

This completes the proof.

Corollary 1.4. Suppose that the hypotheses of Theorem 1.1 hold. Let $\phi$ be an arbitrary weight function with exponential growth rate $\alpha$, where the constant $\alpha>0$ is the same as in Theorem 1.2. Then for any $u_{0}, u_{1} \in \Phi_{b} \cap \Phi_{\phi}$ the following estimates hold:

$$
\begin{gathered}
\left\|S_{t}\left(u_{0}\right)-S_{t}\left(u_{1}\right)\right\|_{\Phi_{\phi}}+\left\|D_{u_{0}} S_{t}\left(u_{0}\right)\left(u_{0}-u_{1}\right)\right\|_{\Phi_{\phi}} \leq C e^{K t}\left\|u_{0}-u_{1}\right\|_{\Phi_{\phi}}, \\
\left\|S_{t}\left(u_{0}\right)-S_{t}\left(u_{1}\right)-D_{u_{0}} S_{t}\left(u_{0}\right)\left(u_{0}-u_{1}\right)\right\|_{\Phi_{\phi}} \leq C e^{2 K t}\left\|u_{0}-u_{1}\right\|\left\|_{\Phi_{b}}\right\| u_{0}-u_{1} \|_{\Phi_{\phi}},
\end{gathered}
$$

where the constant $C$ depends only on the $\Phi_{b}$-norms of the initial conditions $u_{0}$ and $u_{1}$ and on the constant $C_{\phi}$ in (1.24). (It is independent of the explicit form of the weight function $\phi$.)

In fact, multiplying the estimates (1.7) and (1.18) by the weight function $\phi(x)$, taking the supremum over $x \in \mathbb{R}^{n}$, and using the right-hand inequality in (1.28) we obtain (1.29). Estimate (1.30) can be derived in a similar fashion using (1.19).

\section{THE GLOBAL ATtRACTOR OF A NONLINEAR REACTION-DIFFUSION EQUATION AND ITS $\varepsilon$-ENTROPY}

In this section we prove the existence of a (locally compact) global attractor of the semigroup (1.4) and state several upper bounds for the Kolmogorov entropy of this attractor, which is used extensively in what follows.

First we recall that, by contrast with the case of a bounded domain, the semigroup (1.4) generated by equation (0.1) in an unbounded domain such as $\mathbb{R}^{n}$ for example, as a rule has no classical global attractor (see 41 43]). Therefore the following weakened version of the notion of a global attractor is usually used to describe the asymptotic behavior of solutions of evolution equations in unbounded domains as $t \rightarrow \infty$ (see [23, 25, 32]).

Definition 2.1. A set $\mathcal{A} \subset \Phi_{b}$ is called a (locally compact) global attractor of the semigroup (1.4) if the following conditions hold:

1. The set $\mathcal{A}$ is bounded in $\Phi_{b}$ and compact in $\Phi_{\text {loc }}:=L_{\text {loc }}^{\infty}\left(\mathbb{R}^{n}\right)$.

2. The set $\mathcal{A}$ is strictly invariant: $S_{t} \mathcal{A}=\mathcal{A}$.

3. The set $\mathcal{A}$ attracts the images of subsets that are bounded in the phase space $\Phi_{b}$ in the weaker topology of the space $\Phi_{\text {loc }}$. That is, for any $B \subset \Phi_{b}$ that is bounded in $\Phi_{b}$ and any neighborhood $\mathcal{O}(\mathcal{A})$ of the set $\mathcal{A}$ in the local topology of the space $\Phi_{\text {loc }}$ there exists $T=T(B, \mathcal{O})$ such that

$$
S_{t} B \subset \mathcal{O}(\mathcal{A}) \text { for } t \geq T \text {. }
$$


Note that the item 1 in Definition 2.1 means that the restriction $\left.\mathcal{A}\right|_{\Omega}$ of the attractor $\mathcal{A}$ to any bounded domain $\Omega \subset \mathbb{R}^{n}$ is compact in $L^{\infty}(\Omega)$. Further, let $\phi$ be an arbitrary weight function satisfying

$$
\lim _{|x| \rightarrow \infty}|\phi(x)|=0 .
$$

Then, since we have assumed that the attractor $\mathcal{A}$ is bounded (but not compact!) in $\Phi_{b}$, the topologies generated by the embeddings $\mathcal{A} \subset \Phi_{\text {loc }}$ and $\mathcal{A} \subset \Phi_{\phi}$ coincide. Therefore Definition 2.1, item 1 can be reformulated as follows: the set $\mathcal{A}$ is bounded in $\Phi_{b}$ and compact in $\Phi_{\phi}$, for some weight function $\phi$ satisfying (2.2).

Similarly, Definition 2.1, item 3 is equivalent to the following: for any subset $B \subset \Phi_{b}$ that is bounded in $\Phi_{b}$, any bounded domain $\Omega \subset \mathbb{R}^{n}$, and any neighborhood $\mathcal{O}\left(\left.\mathcal{A}\right|_{\Omega}\right)$ of the restriction of the attractor to the domain $\Omega$, in the topology of the space $L^{\infty}(\Omega)$, there exists $T=T(B, \Omega, \mathcal{O})$ such that

$$
\left.\left(S_{t} B\right)\right|_{\Omega} \subset \mathcal{O}\left(\left.\mathcal{A}\right|_{\Omega}\right) \quad \text { for } t \geq T \text {. }
$$

(This means that the time required for the image of the set $B$ restricted to a bounded domain $\Omega$ to enter an $\varepsilon$-neighborhood $\left.\mathcal{O}_{\varepsilon}(\mathcal{A})\right|_{\Omega}$ of the attractor $\mathcal{A}$ (in the topology of $\Phi_{b}$ ) can increase if the domain $\Omega$ is enlarged.)

The following theorem proves that, in the sense of Definition 2.1, the attractor $\mathcal{A}$ exists for the semigroup (1.4).

Theorem 2.1. Suppose that the hypotheses of Theorem 1.1 hold. Then the semigroup (1.4) generated by equation (0.1) has a locally compact attractor $\mathcal{A}$, which is bounded in the space $C_{b}^{4-\delta}\left(\mathbb{R}^{n}\right)$ for any $\delta>0$, is strictly invariant under the action of the extended $(n+1)$-parameter semigroup (1.6), that is,

$$
\mathbb{S}_{(t, h)} \mathcal{A}=\mathcal{A}, \quad t \in \mathbb{R}_{+}, \quad h \in \mathbb{R}^{n},
$$

and admits the following standard description:

$$
\mathcal{A}=\left.\mathcal{K}\right|_{t=0},
$$

where $\mathcal{K}$ is the set of all solutions $u(t)$ of (0.1) that are defined for all $(t, x) \in \mathbb{R}^{n+1}$ and lie in the space $L^{\infty}\left(\mathbb{R}^{n+1}\right)$.

Proof. According to the theorem on the existence of an attractor for abstract semigroups (see, for example, [1, 9, 39]) we need to verify the following conditions:

1. The semigroup $S_{t}$ has an absorbing set $\mathcal{B}$ that is bounded in $\Phi_{b}$ and compact in $\Phi_{\text {loc }}$.

2. The restriction of the operator $S_{t}$ to this compact set is continuous (with respect to the initial conditions) in the topology of the space $\Phi_{\text {loc }}$.

We now verify these conditions. Indeed, according to (1.1) the ball

$$
B:=\left\{u \in \Phi_{b},\|u\|_{\Phi_{b}} \leq 2 C\right\}
$$

is an absorbing set of the semigroup (1.4), which is bounded in $\Phi_{b}$ and closed (but not compact) in $\Phi_{\text {loc }}$. Note also that the restriction of the operator $S_{t}$ to $B$ is continuous for any fixed $t$. Indeed, since $B$ is bounded in $\Phi_{b}$, the local topology on $B$ can be defined by the metric of the space $\Phi_{\phi}$, where $\phi$ is some weight function satisfying (2.2). The fact that $S_{t}$ is continuous (and even Lipschitzian) in this metric is an immediate consequence of (1.29).

We claim that the set $\mathcal{B}:=S_{1} B$ is the required absorbing set. Indeed, by Corollary 1.1 the set $\mathcal{B}$ is bounded in $C_{b}^{4-\delta}\left(\mathbb{R}^{n}\right)$ and is therefore precompact in $\Phi_{\text {loc. }}$. The fact that it is closed in $\Phi_{\text {loc }}$ follows from the continuity of the operator $S_{1}$ on $B$, proved above. Thus, the first condition is verified. The second condition can be proved in the same 
way as the continuity of $S_{t}$ on $B$, since $\mathcal{B}$ is also bounded in $\Phi_{b}$. Consequently, by the abstract theorem on the existence of an attractor mentioned above, the semigroup (1.4) has an attractor $\mathcal{A}$, which is a subset of $\mathcal{B}$ and is therefore bounded in $C_{b}^{4-\delta}\left(\mathbb{R}^{n}\right)$ and admits the standard description (2.5). Finally, the fact that the attractor is invariant under the extended semigroup (1.5) follows immediately from the fact that the original equation (0.1) is spatially homogeneous. Theorem 2.1 is proved.

Thus, equation (0.1) has a global attractor $\mathcal{A}$, which is compact in the local topology of the space $\Phi_{b}$, but, as a rule, is not compact in the topology of the space $\Phi_{b}$. Indeed, suppose that $\mathcal{A}$ is a compact set in $\Phi_{b}$ and let $u_{0}$ be an arbitrary point in it. Then, since $\mathcal{A}$ consists of continuous functions and is invariant under the group $T_{h}$ of spatial translations, the hull

$$
H\left(u_{0}\right):=\left[T_{h} u_{0}, h \in \mathbb{R}^{n}\right]_{C_{b}\left(\mathbb{R}^{n}\right)},
$$

where $[X]_{V}$ denotes the closure of the set $X$ in the space $V$, is a subset of $\mathcal{A}$ and is therefore compact in the space $C_{b}\left(\mathbb{R}^{n}\right)$. Consequently, by the Bochner-Amerio criterion the function $u_{0}(x)$ is almost-periodic with respect to $x$ (that is, $u_{0} \in A P\left(\mathbb{R}^{n}\right)$ ). Thus, if $\mathcal{A}$ is compact in the uniform topology, this gives the embedding

$$
\mathcal{A} \subset A P\left(\mathbb{R}^{n}\right) .
$$

Since every equilibrium automatically belongs to the attractor, it follows from (2.6) that, in particular, all the bounded solutions of the elliptic equation

$$
a \Delta_{x} u-\left(\vec{L}, \nabla_{x}\right) u-f(u)=0, \quad x \in \mathbb{R}^{n},
$$

must be almost periodic with respect to $x \in \mathbb{R}^{n}$. Note that this condition is extremely restrictive and, as a rule, does not hold for quite realistic examples of equations of the form (0.1). For instance, consider the following scalar Chafee-Infante equation in $\mathbb{R}^{n}$ :

$$
\partial_{t} u=\Delta_{x} u+u-u^{3} .
$$

It is obvious that this equation satisfies all the hypotheses of Theorem 1.1, and so there exists a locally compact attractor $\mathcal{A}$. On the other hand, the function $u_{0}(x)=u_{0}\left(x_{1}\right):=$ $\tanh \left(\frac{x_{1}}{\sqrt{2}}\right)$ is an equilibrium of this equation, which is not almost-periodic. Thus, $\mathcal{A}$ is not compact in the original phase space $\Phi_{b}$.

The following example shows that solutions of (0.1) in general are not attracted to $\mathcal{A}$ in the uniform topology of the space $\Phi_{b}$, even in the case where $\mathcal{A}$ is compact in $\Phi_{b}$. Consider the scalar equation

$$
\partial_{t} u=\Delta_{x} u-f(u), \quad \text { where } f(u)= \begin{cases}(u+1)^{3} & \text { for } u<-1 \\ 0 & \text { for } u \in[-1,1] \\ (u-1)^{3} & \text { for } u>1\end{cases}
$$

It is obvious that (2.9) satisfies all the hypotheses of Theorem 1.1. Moreover, using spatially homogeneous solutions of this equation as barrier functions one can easily show that $\|\mathcal{A}\|_{C_{b}\left(\mathbb{R}^{n}\right)} \leq 1$ and therefore every bounded solution $u(t, x) \in \mathcal{K}$ of $(2.9)$ is a bounded solution of a linear heat equation in $\mathbb{R}^{n}$. Thus, according to Liouville's theorem we have

$$
\mathcal{A}:=\left\{u_{0}(x) \equiv u_{0}, u_{0} \in[-1,1]\right\} .
$$

Therefore, $\mathcal{A}$ is compact in the uniform topology of $\Phi_{b}$. On the other hand, consider the solution $u(t):=S_{t} u_{0}$ of (2.9) with the initial condition $u_{0}(x)=\operatorname{sgn}\left(x_{1}\right) \in \Phi_{b}$. Since the $u_{ \pm}(x) \equiv \pm 1$ are equilibria of the system under consideration, using (1.7) gives

$$
\lim _{x_{1} \rightarrow \pm \infty} u(t, x)= \pm 1 \quad \text { for any finite } t \geq 0
$$


and therefore

$$
\operatorname{dist}_{L^{\infty}\left(\mathbb{R}^{n}\right)}(u(t), \mathcal{A})=1 \quad \text { for any finite } t .
$$

Equations (2.8) and (2.9) show that it is necessary to use the weakened version of the notion of a global attractor (see Definition 2.1) to investigate equations of the form (0.1) in unbounded domains. Another fundamental distinction between the theory of attractors of evolutionary equations in unbounded domains and the classical theory of attractors of these equations in bounded domains (see [1, 9, 39]) is the fact that $\mathcal{A}$ is infinite-dimensional; both the Hausdorff and the fractal dimensions of the restriction $\left.\mathcal{A}\right|_{\Omega}$ of the attractor to any bounded domain, as a rule, turn out to be infinite; see [3, 14, 23, 24. Therefore, in order to obtain quantitative and qualitative information on such attractors the notion of Kolmogorov's $\varepsilon$-entropy is usually used (see [3, 4, 9, 19, 20, 21, 23, 24, 41, 42, 43, 44]).

Definition 2.2. Let $K$ be a precompact set in a metric space $(M, d)$. For any $\varepsilon>0$ this set can be covered by finitely many $\varepsilon$-balls in $M$. Let $N_{\varepsilon}(K, M)$ be the least possible number of such balls. The Kolmogorov $\varepsilon$-entropy of the set $K$ is defined to be the number

$$
\mathbb{H}_{\varepsilon}(K, M)=\mathbb{H}_{\varepsilon}(K, d):=\ln N_{\varepsilon}(K, M) .
$$

We recall that the fractal dimension of $K$ can be expressed in terms of the $\varepsilon$-entropy by the formula

$$
\operatorname{dim}_{F}(K, M):=\lim \sup _{\varepsilon \rightarrow 0} \frac{\mathbb{H}_{\varepsilon}(K, M)}{\ln \frac{1}{\varepsilon}}
$$

(see, for example, [7] or [40]).

The next theorem gives the typical form of the asymptotics of the $\varepsilon$-entropy of the restriction $\left.\mathcal{A}\right|_{B_{x_{0}}^{R}}$ of $\mathcal{A}$ to the ball $B_{x_{0}}^{R}$ with radius $R$ and center at the point $x_{0} \in \mathbb{R}^{n}$.

Theorem 2.2. Suppose that the hypotheses of Theorem 1.1 hold. Then the following estimate holds:

$$
\mathbb{H}_{\varepsilon}\left(\left.\mathcal{A}\right|_{B_{x_{0}}^{R}}, L^{\infty}\left(B_{x_{0}}^{R}\right)\right) \leq C\left(R+\ln _{+} \frac{R_{0}}{\varepsilon}\right)^{n} \ln _{+} \frac{R_{0}}{\varepsilon},
$$

where $\ln _{+} z:=\max \{0, \ln z\}$ and the constants $C$ and $R_{0}$ are independent of $\varepsilon, R$, and $x_{0}$.

Scheme of the proof. For each $x_{0} \in \mathbb{R}^{n}$ and $R \in \mathbb{R}_{+}$consider the weight function

$$
\phi_{R, x_{0}}(x):=e^{-\alpha \operatorname{dist}_{\mathbb{R}} n\left(x, B_{x_{0}}^{R}\right)},
$$

where $\alpha>0$ is the same as in Theorem 1.2. Then by Proposition 1.2 the functions (2.14) are weight functions with exponential growth rate $\alpha$ (with $C_{\phi_{R, x_{0}}} \equiv 1$, which is independent of $R$ and $x_{0}$ ). Hence by Theorem 1.2, Corollary 1.2, and (1.28)

$$
\left\|S_{1} u_{1}-S_{1} u_{2}\right\|_{W_{\phi_{R}, x_{0}}^{1, \infty}\left(\mathbb{R}^{n}\right)} \leq L\left\|u_{1}-u_{2}\right\|_{L_{\phi_{R, x_{0}}}^{\infty}}\left(\mathbb{R}^{n}\right) \quad \forall u_{1}, u_{2} \in \mathcal{A},
$$

where $S_{1}$ is the solution operator of problem (0.1) per unit time,

$$
W_{\phi}^{1, \infty}\left(\mathbb{R}^{n}\right):=\left\{u \in L_{\phi}^{\infty}\left(\mathbb{R}^{n}\right): \nabla_{x} u \in L_{\phi}^{\infty}\left(\mathbb{R}^{n}\right)\right\},
$$

the constant $L$ is independent of $x_{0} \in \mathbb{R}^{n}, R \in \mathbb{R}_{+}$, and $u_{1}, u_{2} \in \mathcal{A}$.

Note that $\phi_{R, x_{0}}(x) \equiv 1$ if $x \in B_{x_{0}}^{R}$. Hence,

$$
\mathbb{H}_{\varepsilon}\left(\left.\mathcal{A}\right|_{B_{x_{0}}^{R}}, L^{\infty}\left(B_{x_{0}}^{R}\right)\right) \leq \mathbb{H}_{\varepsilon}\left(\mathcal{A}, L_{\phi_{R, x_{0}}}^{\infty}\left(\mathbb{R}^{n}\right)\right) .
$$

Thus, in order to prove (2.13) it is sufficient to estimate the $\varepsilon$-entropy of the attractor in the weighted space $L_{\phi_{R, x_{0}}}^{\infty}\left(\mathbb{R}^{n}\right)$. For this we need the following lemma. 
Lemma 2.1. The following recurrence estimate holds:

$$
\mathbb{H}_{\varepsilon / 2}\left(\mathcal{A}, L_{\phi_{R, x_{0}}}^{\infty}\left(\mathbb{R}^{n}\right)\right) \leq C\left(R+\ln _{+} \frac{R_{0}}{\varepsilon}\right)^{n}+\mathbb{H}_{\varepsilon}\left(\mathcal{A}, L_{\phi_{R, x_{0}}}^{\infty}\left(\mathbb{R}^{n}\right)\right),
$$

where the constants $C$ and $R_{0}$ are independent of $\varepsilon, R$, and $x_{0}$.

Proof. Suppose that $\left\{u_{1}, \ldots, u_{k}, k=1, \ldots, N(\varepsilon)\right\}$ is an arbitrary covering of $\mathcal{A}$ by $\varepsilon$-balls $B\left(\varepsilon, u_{i}, L_{\phi_{R, x_{0}}}^{\infty}\right)$ in the space $L_{\phi_{R, x_{0}}}^{\infty}$ with centers $u_{i} \in \mathcal{A}$. Then since $\mathcal{A}$ is invariant under $S_{t}$ and (2.15) holds, thus the system of $L \varepsilon$-balls in the space $W_{\phi_{R, x_{0}}}^{1, \infty}$ with centers at the points $S_{1} u_{i}, \quad i=1, \ldots, N(\varepsilon)$, also covers $\mathcal{A}$. Now covering each set $\mathcal{A} \cap B\left(L \varepsilon, S_{1} u_{i}, W_{\phi_{R, x_{0}}}^{1, \infty}\right)$ by $\frac{\varepsilon}{2}$-balls in the space $L_{\phi_{R}, x_{0}}^{\infty}$ we obtain a new $\frac{\varepsilon}{2}$-covering of $\mathcal{A}$ with the number of balls satisfying

$$
\begin{aligned}
N\left(\frac{\varepsilon}{2}\right) & \leq M(\varepsilon) N(\varepsilon), \\
\text { where } M(\varepsilon) & :=\max _{i=1, \ldots, N(\varepsilon)} N_{\varepsilon / 2}\left(\mathcal{A} \cap B\left(L \varepsilon, S_{1} u_{i}, W_{\phi_{R, x_{0}}}^{1, \infty}\right), L_{\phi_{R, x_{0}}}^{\infty}\right) .
\end{aligned}
$$

(The quantity $M(\varepsilon)$ is finite, since $\mathcal{A}$ is a compact set in $L_{\phi_{R, x_{0}}}^{\infty}$.) To estimate $M(\varepsilon)$ we observe that

$$
\left\|\left.\mathcal{A}\right|_{\mathbb{R}^{n} \backslash B_{x_{0}}^{R+R(\varepsilon)}}\right\|_{L_{\phi_{R, x_{0}}}^{\infty}\left(\mathbb{R}^{n} \backslash B_{x_{0}}^{R+R(\varepsilon)}\right)} \leq \frac{\varepsilon}{2}
$$

where $R(\varepsilon):=\alpha^{-1} \ln _{+} \frac{R_{0}}{\varepsilon}$ and $R_{0}:=2\|\mathcal{A}\|_{\Phi_{b}}$. Hence,

$$
\begin{aligned}
N_{\varepsilon / 2}(\mathcal{A} \cap & \left.B\left(L \varepsilon, S_{1} u_{i}, W_{\phi_{R, x_{0}}}^{1, \infty}\right), L_{\phi_{R, x_{0}}}^{\infty}\right) \\
\leq & N_{\varepsilon / 2}\left(B\left(L \varepsilon, S_{1} u_{i}, W_{\phi_{R, x_{0}}}^{1, \infty}\right), L_{\phi_{R, x_{0}}}^{\infty}\left(B_{x_{0}}^{R+R(\varepsilon)}\right)\right) \\
\leq & N_{1 / 2 L}\left(B\left(1,0, W_{\phi_{R, x_{0}}}^{1, \infty}\left(B_{x_{0}}^{R+R(\varepsilon)}\right)\right), L_{\phi_{R, x_{0}}}^{\infty}\left(B_{x_{0}}^{R+R(\varepsilon)}\right)\right) .
\end{aligned}
$$

(The right-hand side of this inequality makes sense, since $W_{\phi}^{1, \infty}(V) \subset L_{\phi}^{\infty}(V)$ is a compact embedding for any bounded domain $V$ and any weight function $\phi$.) Moreover, since the right-hand side of this inequality is independent of $i$,

$$
\ln M(\varepsilon) \leq \mathbb{H}_{1 /(2 L)}\left(B\left(1,0, W_{\phi_{R, x_{0}}}^{1, \infty}\left(B_{x_{0}}^{R+R(\varepsilon)}\right)\right), L_{\phi_{R, x_{0}}}^{\infty}\left(B_{x_{0}}^{R+R(\varepsilon)}\right)\right) .
$$

Therefore to complete the proof of the lemma it is sufficient to estimate the $\frac{1}{2 L}$-entropy of the embedding of the unit ball in $W_{\phi_{R, x_{0}}}^{1, \infty}\left(B_{x_{0}}^{R+R(\varepsilon)}\right)$ into the space $L_{\phi_{R, x_{0}}}^{\infty}\left(B_{x_{0}}^{R+R(\varepsilon)}\right)$. For that we observe that the mapping $F u:=\phi_{R, x_{0}}^{-1} u$ realizes an isomorphism of the Banach pairs $\left(L^{\infty}\left(B_{x_{0}}^{M}\right), W^{1, \infty}\left(B_{x_{0}}^{M}\right)\right)$ and $\left(L_{\phi_{R}, x_{0}}^{\infty}\left(B_{x_{0}}^{M}\right), W_{\phi_{R, x_{0}}}^{1, \infty}\left(B_{x_{0}}^{M}\right)\right)$ for any $M>0$. Moreover, it is easy to show that

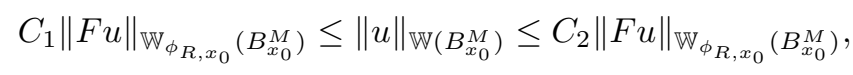

where $\mathbb{W}=L^{\infty}$ or $\mathbb{W}=W^{1, \infty}$ and the constants $C_{1}$ and $C_{2}$ are independent of $x_{0}, R$, and $M$; see [41]. Hence,

$$
\ln M(\varepsilon) \leq \mathbb{H}_{1 / L L^{\prime}}\left(B\left(1,0, W^{1, \infty}\left(B_{x_{0}}^{R+R(\varepsilon)}\right)\right), L^{\infty}\left(B_{x_{0}}^{R+R(\varepsilon)}\right)\right),
$$

where the constant $L^{\prime}$ depends only on $C_{1}$ and $C_{2}$. Since the Kolmogorov entropy is subadditive in the metric of $L^{\infty}$ (see Proposition 3.2 below), it is easy to show that the 
inequality

$$
\begin{aligned}
M(\varepsilon) & \leq C^{\prime}(R+R(\varepsilon))^{n} \mathbb{H}_{1 /\left(L L^{\prime}\right)}\left(B\left(1,0, W^{1, \infty}\left(B_{0}^{1}\right)\right), L^{\infty}\left(B_{0}^{1}\right)\right) \\
& \leq C^{\prime \prime}\left(R+\ln _{+} \frac{R_{0}}{\varepsilon}\right)^{n}
\end{aligned}
$$

follows from (2.21), with $C^{\prime \prime}$ independent of $\varepsilon, x_{0}$, and $R$. Together (2.18) and (2.22) complete the proof of Lemma 2.1.

It is now easy to complete the proof of the theorem. Indeed, since $\mathbb{H}_{R_{0}}\left(\mathcal{A}, L_{\phi_{R, x_{0}}}^{\infty}\right)=0$ for any $R$ and $x_{0}$ (recall that $\|\mathcal{A}\|_{L^{\infty}} \leq R_{0}$ ), by iterating estimate $(2.17) \quad k$ times we obtain

$$
\mathbb{H}_{R_{0} / 2^{k}}\left(\mathcal{A}, L_{\phi_{R, x_{0}}}^{\infty}\right) \leq C_{1}(R+k)^{n} k,
$$

where $C_{1}$ is independent of $k$. Choosing $k$ so that $\varepsilon \sim R_{0} 2^{-k}$ we now obtain

$$
\mathbb{H}_{\varepsilon}\left(\mathcal{A}, L_{\phi_{R, x_{0}}}^{\infty}\right) \leq C\left(R+\ln _{+} \frac{R_{0}}{\varepsilon}\right)^{n} \ln _{+} \frac{R_{0}}{\varepsilon},
$$

which completes the proof of Theorem 2.2.

Remark 2.1. A universal estimate of the form (2.13), which is a standard consequence of the smoothing estimate (1.16) for the differences between solutions lying in the attractor, was obtained in [3] for the case of reaction-diffusion equations in $\mathbb{R}^{n}$, and in [41] for the case of autonomous and nonautonomous equations of the form (0.1) in an arbitrary unbounded domain. It was also shown there that this estimate is, in a sense, sharp for all values of the parameters $\varepsilon, R$, and $x_{0}$ (see also $\S 7$ below). In the special case of the Landau-Ginzburg equation in $\mathbb{R}^{n}$ for $n<3$ and $R \gg\left(\frac{1}{\varepsilon}\right)^{n}$ this estimate was also proved in [19].

To conclude this section we obtain one important corollary of Theorem 2.2, which will allow us to prove that several dynamic characteristics of $\mathcal{A}$ introduced in the next two sections are finite.

Corollary 2.1. Suppose that the hypotheses of Theorem 2.2 hold and let $\mathcal{K} \subset L^{\infty}\left(\mathbb{R}^{n+1}\right)$ be the set of all bounded solutions of (0.1) defined in Theorem 2.1. Then for any $L>0$

$$
\mathbb{H}_{\varepsilon}\left(\left.\mathcal{K}\right|_{\left[0, L \ln \frac{1}{\varepsilon}\right]^{n+1}}, L^{\infty}\left(\left[0, L \ln \frac{1}{\varepsilon}\right]^{n+1}\right)\right) \leq C_{L}\left(\ln _{+} \frac{R_{0}^{\prime}}{\varepsilon}\right)^{n+1},
$$

where the constants $C_{L}$ and $R_{0}^{\prime}$ depend on $L$ but are independent of $\varepsilon>0$.

Proof. We introduce the weight function

$$
\varphi_{\varepsilon, L}(x):=\sup _{y \in\left[0, L \ln \frac{1}{\varepsilon}\right]^{n}} e^{-\alpha|x-y|},
$$

where $\alpha>0$ is the same as in Theorem 1.2. Then, since the attractor $\mathcal{A}$ is bounded in $L^{\infty}\left(\mathbb{R}^{n}\right)$, by $(2.13)$ we have

$$
\begin{aligned}
\mathbb{H}_{\varepsilon}\left(\mathcal{A}, L_{\varphi_{\varepsilon}, L}^{\infty}\left(\mathbb{R}^{n}\right)\right) & \leq \mathbb{H}_{\varepsilon}\left(\left.\mathcal{A}\right|_{\left[-\alpha^{-1} \ln \frac{C}{\varepsilon}, L \ln \frac{1}{\varepsilon}+\alpha^{-1} \ln \frac{C}{\varepsilon}\right]^{n}}, L^{\infty}\left(\mathbb{R}^{n}\right)\right) \\
& \leq C_{L}\left(\ln \frac{R_{0}^{\prime}}{\varepsilon}\right)^{n+1}
\end{aligned}
$$

for some constants $C:=\|\mathcal{A}\|_{\Phi_{b}}, C_{L}$, and $R_{0}^{\prime}$ that are independent of $\varepsilon$, where $1 \geq \varepsilon>0$.

On the other hand, by Proposition 1.2 the function (2.26) is a weight function with exponential growth rate $\alpha$ where $C_{\phi_{\varepsilon, L}} \equiv 1$. Therefore it follows from (1.29) that

$$
\left\|S_{t} u_{0}-S_{t} u_{1}\right\|_{L_{\varphi_{\varepsilon, L}}^{\infty}\left(\mathbb{R}^{n}\right)} \leq C e^{K t}\left\|u_{0}-u_{1}\right\|_{L_{\varphi_{\varepsilon, L}}^{\infty}\left(\mathbb{R}^{n}\right)} \quad \forall u_{0}, u_{1} \in \mathcal{A},
$$


where the constant $C$ is independent of $\varepsilon$ and $L$. Thus,

$$
\begin{aligned}
\mathbb{H}_{\varepsilon}\left(\left.\mathcal{K}\right|_{\left[0, L \ln \frac{1}{\varepsilon}\right]^{n+1}}, L^{\infty}\left(\mathbb{R}^{n+1}\right)\right) & \leq \mathbb{H}_{\varepsilon}\left(\left.\mathcal{K}\right|_{\left[0, L \ln \frac{1}{\varepsilon}\right] \times \mathbb{R}^{n}}, L_{\varphi_{\varepsilon, L}}^{\infty}\left(\mathbb{R}^{n+1}\right)\right) \\
& \leq \mathbb{H}_{C^{-1} \varepsilon e^{-K L \ln 1 / \varepsilon}}\left(\mathcal{A}, L_{\varphi_{\varepsilon, L}}^{\infty}\left(\mathbb{R}^{n}\right)\right) \\
& =\mathbb{H}_{C^{-1} \varepsilon^{K L+1}}\left(\mathcal{A}, L_{\varphi_{\varepsilon, L}}^{\infty}\left(\mathbb{R}^{n}\right)\right) \\
& \leq C_{L}^{\prime}\left(\ln \frac{R_{0}^{\prime \prime}}{\varepsilon}\right)^{n+1} .
\end{aligned}
$$

Corollary 2.1 is proved.

\section{The SPACE-Time topological Entropy of the attractor}

Now we begin to investigate the space-time dynamics generated by (0.1). Following [44] we interpret the multiparameter semigroup (1.6) as a dynamical system with multidimensional time, acting on the attractor $\mathcal{A}$. We shall describe the space-time structure of solutions of (0.1) using dynamic characteristics of the semigroup (1.6) restricted to the attractor. In this section we introduce and study the topological entropy of the action of the multiparameter semigroup (1.6) on the attractor. We begin by recalling one of the possible definitions of the topological entropy (see, for example, [6] and the references therein for a more detailed study of this notion).

Definition 3.1. Fix some (arbitrary) weight function satisfying (2.2) and the corresponding weighted space $\Phi_{\phi}=L_{\phi}\left(\mathbb{R}^{n}\right)$. Then by Theorem 2.1 the set

$$
\left(\mathcal{A}, d_{\phi}\right), \quad d_{\phi}\left(u_{1}, u_{2}\right):=\left\|u_{1}-u_{2}\right\|_{L_{\phi}^{\infty}\left(\mathbb{R}^{n}\right)},
$$

is a compact metric space. For each $R>0$ define a new equivalent metric on $\mathcal{A}$ by

$$
d_{\phi}^{R}\left(u_{1}, u_{2}\right):=\sup _{(t, h) \in[0, R]^{n+1}} d_{\phi}\left(S_{(t, h)} u_{1}-S_{(t, h)} u_{2}\right), \quad u_{1}, u_{2} \in \mathcal{A} .
$$

Since $\left(\mathcal{A}, d_{\phi}^{R}\right)$ is a compact metric space, the Kolmogorov $\varepsilon$-entropy $\mathbb{H}_{\varepsilon}\left(\mathcal{A}, d_{\phi}^{R}\right)$ is well defined for any $\varepsilon>0$. By definition, the space-time topological entropy of $\mathcal{A}$ is the topological entropy of the action of the semigroup (1.6) on $\mathcal{A}$, which is defined as follows:

$$
h_{\mathrm{top}}(\mathcal{A}):=h_{\mathrm{top}}\left(\mathcal{A}, \mathbb{S}_{(t, h)}\right)=\lim _{\varepsilon \rightarrow 0} \limsup _{R \rightarrow \infty} \frac{1}{R^{n+1}} \mathbb{H}_{\varepsilon}\left(\mathcal{A}, d_{\phi}^{R}\right) .
$$

Recall that the topological entropy (3.3) depends only on the topology in $\mathcal{A}$, but not on the choice of the metric that defines it (see [6]). In particular, (3.3) does not depend on the choice of the weight function $\phi$ in (3.1). Hence the metric in Definition 3.1 is well defined. As the next step we obtain more convenient formulae for calculating the quantity (3.3). To do this we need the notion of the mean Kolmogorov $\varepsilon$-entropy (see [7]).

Definition 3.2. Let $\mathcal{K}$ be a locally compact set in $L^{\infty}\left(\mathbb{R}^{n+1}\right)$. Then the mean $\varepsilon$-entropy of $\mathcal{K}$ in $L^{\infty}$ is the number

$$
\overline{\mathbb{H}}_{\varepsilon}\left(\mathcal{K}, L^{\infty}\left(\mathbb{R}^{n+1}\right)\right):=\limsup _{R \rightarrow \infty} \frac{1}{R^{n+1}} \mathbb{H}_{\mathcal{E}}\left(\left.\mathcal{K}\right|_{[0, R]^{n+1}}, L^{\infty}\left([0, R]^{n+1}\right)\right) .
$$

Of course, it is more natural to consider the restrictions of $\mathcal{K}$ to the intervals $\left[-\frac{R}{2}, \frac{R}{2}\right]^{n+1}$, but in what follows we consider only sets $\mathcal{K}$ that are invariant under the group of "spacetime" translations in $\mathbb{R}^{n+1}$; therefore this distinction is not important.

The following proposition gives an expression for (3.3) in terms of the mean $\varepsilon$-entropy.

Proposition 3.1. Let $\mathcal{K}$ be the set of all bounded solutions of (0.1) defined in Theorem 2.1. Then (3.3) can be calculated using the formula

$$
h_{\text {top }}\left(\mathcal{A}, \mathbb{S}_{(t, h)}\right)=\lim _{\varepsilon \rightarrow 0} \overline{\mathbb{H}}_{\varepsilon}\left(\mathcal{K}, L^{\infty}\left(\mathbb{R}^{n+1}\right)\right) .
$$


Proof. From the definition of $\mathcal{A}$ and (3.3) it follows that

$$
\mathbb{H}_{\varepsilon}\left(\mathcal{A}, d_{\phi}^{R}\right)=\mathbb{H}_{\varepsilon}\left(\left.\mathcal{K}\right|_{[0, R] \times \mathbb{R}^{n}}, L_{\phi_{R}}^{\infty}\left([0, R] \times \mathbb{R}^{n}\right)\right),
$$

where $\phi_{R}(x):=\sup _{y \in[0, R]^{n}} \phi(x-y)$. We now observe that, on the one hand,

$$
\phi_{R}(x) \geq \phi(0)=c_{0}>0 \quad \text { for } \quad x \in[0, R]^{n},
$$

and, on the other hand, it follows from condition (2.2) that for any $\varepsilon>0$ there exists $R(\varepsilon)$ (depending on $\varepsilon$ but independent of $R$ ) such that

$$
\phi_{R}(x) \leq \frac{\varepsilon}{\|\mathcal{K}\|_{L^{\infty}\left(\mathbb{R}^{n+1}\right)}} \quad \text { for } x \notin[-R(\varepsilon), R+R(\varepsilon)]^{n} .
$$

Using inequalities (3.7) and (3.8) we find

$$
\begin{aligned}
\mathbb{H}_{\varepsilon / c_{0}}\left(\left.\mathcal{K}\right|_{[0, R]^{n+1}}, L^{\infty}\left([0, R]^{n+1}\right)\right) \\
\quad \leq \mathbb{H}_{\varepsilon}\left(\left.\mathcal{K}\right|_{[0, R] \times \mathbb{R}^{n}}, L_{\phi_{R}}^{\infty}\left([0, R] \times \mathbb{R}^{n}\right)\right) \\
\quad \leq \mathbb{H}_{\varepsilon / C_{0}}\left(\left.\mathcal{K}\right|_{[0, R+2 R(\varepsilon)]^{n+1}}, L^{\infty}\left([0, R+2 R(\varepsilon)]^{n+1}\right)\right),
\end{aligned}
$$

where $C_{0}:=\sup _{x \in \mathbb{R}^{n}} \phi(x)$. We divide inequality (3.9) by $R^{n+1}$, pass to the limit as $R \rightarrow \infty$, and use (3.6) and the fact that $L(\varepsilon)$ is independent of $R$ to obtain

$$
\overline{\mathbb{H}}_{\varepsilon / c_{0}}\left(\mathcal{K}, L^{\infty}\left(\mathbb{R}^{n+1}\right)\right) \leq \limsup _{R \rightarrow \infty} \frac{1}{R^{n+1}} \mathbb{H}_{\varepsilon}\left(\mathcal{A}, d_{\phi}^{R}\right) \leq \overline{\mathbb{H}}_{\varepsilon / C_{0}}\left(\mathcal{K}, L^{\infty}\left(\mathbb{R}^{n+1}\right)\right) .
$$

Passing to the limit as $\varepsilon \rightarrow 0$ in (3.10) we obtain (3.5). Proposition 3.1 is proved.

The subadditivity property of the Kolmogorov entropy in the $L^{\infty}$-metric plays a key role as we investigate the topological entropy (3.3) and its generalizations further.

Proposition 3.2. Let $V_{1}$ and $V_{2}$ be two subdomains in $\mathbb{R}^{M}$ and let $V=V_{1} \cup V_{2}$. Suppose also that $K$ is a precompact subset in $L^{\infty}(V)$. Then

$$
\mathbb{H}_{\varepsilon}\left(K, L^{\infty}(V)\right) \leq \mathbb{H}_{\varepsilon}\left(\left.K\right|_{V_{1}}, L^{\infty}\left(V_{1}\right)\right)+\mathbb{H}_{\varepsilon}\left(\left.K\right|_{V_{2}}, L^{\infty}\left(V_{2}\right)\right) .
$$

Indeed, let $\left\{v_{i}, i=1, \ldots, N_{1}\right\}$ and $\left\{w_{j}, j=1, \ldots, N_{2}\right\}$ be the centers of $\varepsilon$-balls in minimal coverings of $\left.K\right|_{V_{1}}$ and $\left.K\right|_{V_{2}}$. Then the system of $\varepsilon$-balls with centers at the points

$$
\begin{aligned}
& V_{i j} \in L^{\infty}(V), \\
& V_{i j}(x)=\left\{\begin{array}{ll}
v_{i}(x), & x \in V_{1}, \\
w_{j}(x), & x \in V \backslash V_{1},
\end{array} \quad i=1, \ldots, N_{1}, \quad j=1, \ldots, N_{2},\right.
\end{aligned}
$$

covers the whole set $K$, which gives (3.11).

Remark 3.1. Generally speaking, in a more formal exposition of the theory of the Kolmogorov $\varepsilon$-entropy one has to distinguish between the $\varepsilon$-entropy $\mathbb{H}_{\varepsilon}^{\mathrm{int}}(K, M)$ calculated with respect to $\varepsilon$-coverings of $K$ by balls centered in $K$ and the $\varepsilon$-entropy $\mathbb{H}_{\varepsilon}^{\operatorname{ext}}(K, M)$ defined by using $\varepsilon$-balls with centers not necessarily in $K$. For instance, to derive (3.6) it is natural to use $\mathbb{H}_{\varepsilon}^{\text {int }}$, while the subadditive estimate $(3.11)$ is valid only for $\mathbb{H}_{\varepsilon}^{\text {ext }}$. However, it is clear that

$$
\mathbb{H}_{\varepsilon}^{\text {ext }}(K, M) \leq \mathbb{H}_{\varepsilon}^{\mathrm{int}}(K, M) \leq \mathbb{H}_{\varepsilon / 2}^{\text {ext }}(K, M),
$$

which renders the distinction between these entropies completely irrelevant in the theory presented here. Therefore, by abusing the notation, we use one and the same symbol $\mathbb{H}_{\varepsilon}(K, M)$ for both $\varepsilon$-entropies. 
Corollary 3.1. The function

$$
\Phi_{\mathcal{K}}\left(R_{1}, \ldots, R_{n}\right):=\mathbb{H}_{\varepsilon}\left(\left.\mathcal{K}\right|_{\left[0, R_{1}\right] \times \cdots \times\left[0, R_{n}\right]}, L^{\infty}\left(\mathbb{R}^{n+1}\right)\right):\left(\mathbb{R}_{+}\right)^{n+1} \rightarrow \mathbb{R}_{+}
$$

is subadditive in each variable and therefore

$$
\begin{aligned}
\overline{\mathbb{H}}_{\varepsilon}\left(\mathcal{K}, L^{\infty}\left(\mathbb{R}^{n+1}\right)\right) & =\lim _{R_{i} \rightarrow \infty, i=1, \ldots, n} \Phi_{\mathcal{K}}\left(R_{1}, \ldots, R_{n}\right) \\
& =\inf _{R_{i} \in \mathbb{R}_{+}, i=1, \ldots, n} \Phi_{\mathcal{K}}\left(R_{1}, \ldots, R_{n}\right) .
\end{aligned}
$$

The subadditivity of the function defined in (3.13) follows immediately from (3.11), and both the invariance of $\mathcal{K}$ under space-time translations and formula (3.14) are standard consequences of the subadditivity property.

Remark 3.2. Formula (3.5) and the existence of the repeated limit (3.14) give another equivalent definition of (3.3), namely,

$$
h_{\mathrm{top}}\left(\mathcal{A}, \mathbb{S}_{(t, h)}\right)=\lim _{\varepsilon \rightarrow 0} \lim _{T \rightarrow \infty} \frac{1}{T} \lim _{R \rightarrow \infty} \frac{1}{(2 R)^{n}} \mathbb{H}_{\varepsilon}\left(\left.\mathcal{K}\right|_{[0, T] \times[-R, R]^{n}}, L^{\infty}\left(\mathbb{R}^{n+1}\right)\right),
$$

which coincides with the definition of the so-called topological entropy per unit volume introduced in [20].

Now it is easy to verify that (3.3) is finite for the space-time dynamics generated by equation (0.1).

Corollary 3.2. Suppose that the hypotheses of Theorem 1.1 hold. Then the space-time topological entropy of the attractor is finite:

$$
h_{\text {top }}\left(\mathcal{A}, \mathbb{S}_{(t, h)}\right) \leq C<\infty .
$$

In fact, using the subadditivity of the function (3.13) and estimate (2.25) we see that for $R>\ln \frac{1}{\varepsilon}$

$$
\begin{aligned}
H_{\varepsilon}\left(\left.\mathcal{K}\right|_{[0, R]^{n+1}}, L^{\infty}\left(\mathbb{R}^{n+1}\right)\right) & \leq\left(\frac{R}{\ln \frac{1}{\varepsilon}}+1\right)^{n+1} \mathbb{H}_{\varepsilon}\left(\mathcal{K}_{\left[0, \ln \frac{1}{\varepsilon}\right]^{n+1}}, L^{\infty}\left(\mathbb{R}^{n+1}\right)\right) \\
& \leq C\left[R^{n+1}+R^{n}\left(\ln \frac{1}{\varepsilon}\right)^{n+1}\right] .
\end{aligned}
$$

Substituting (3.17) into (3.4) and (3.5) we obtain (3.16).

The next theorem shows that when calculating the quantity $\overline{\mathbb{H}}_{\varepsilon}(\mathcal{K})$ (and therefore $h_{\text {top }}(\mathcal{A})$ ) we can use not only the cubes $R \cdot[0,1]^{n+1}$ for "windows", but also arbitrary bounded domains $R \cdot \Omega, R \in \mathbb{R}_{+}$(see (3.4) and (3.18)).

Theorem 3.1. Let $\Omega$ be an arbitrary bounded open set in $\mathbb{R}^{n+1}$ with piecewise smooth boundary. Then for any $\varepsilon>0$

$$
\overline{\mathbb{H}}_{\varepsilon}\left(\mathcal{K}, L^{\infty}\left(\mathbb{R}^{n}\right)\right)=\frac{1}{\operatorname{vol}(\Omega)} \lim _{\varepsilon \rightarrow 0} \lim _{R \rightarrow \infty} \frac{1}{R^{n+1}} \mathbb{H}_{\varepsilon}\left(\left.\mathcal{K}\right|_{R \cdot \Omega}, L^{\infty}(R \cdot \Omega)\right),
$$

where $\operatorname{vol}(\Omega)$ is the Lebesgue measure of the set $\Omega$ in $\mathbb{R}^{n+1}$.

Proof. Since $\Omega$ is bounded, we can assume without loss of generality that $\bar{\Omega} \subset(0,1)^{n+1}$. For any $M \in \mathbb{N}$ we consider the lattice $M^{-1} \mathbb{Z}^{n+1}$ and the corresponding covering of the space $\mathbb{R}^{n+1}$ by the cubes $\mathcal{C}_{l}^{M}:=l+M^{-1}[0,1]^{n+1}, l \in M^{-1} \mathbb{Z}^{n+1}$. We also introduce the following sets:

$$
\mathbb{Z}^{M}(\Omega):=\left\{l \in M^{-1} \mathbb{Z}^{n+1}, \mathcal{C}_{l}^{M} \cap \Omega \neq \varnothing\right\}, \quad \Omega_{M}:=\bigcup_{l \in \mathbb{Z}^{M}(\Omega)} \mathcal{C}_{l}^{M} .
$$


Then the embedding $\Omega \subset \Omega_{M}$, the invariance of $\mathcal{K}$ under space-time translations, and the subadditivity (3.11) together yield

$$
\begin{aligned}
\mathbb{H}_{\varepsilon}\left(\left.\mathcal{K}\right|_{R \cdot \Omega}\right) & \leq \mathbb{H}_{\varepsilon}\left(\left.\mathcal{K}\right|_{R \cdot \Omega_{M}}\right) \\
& \leq \sum_{l \in R \cdot \mathbb{Z}^{M}(\Omega)} \mathbb{H}_{\mathcal{\varepsilon}}\left(\left.\mathcal{K}\right|_{\mathcal{C}_{l}^{R M}}\right) \\
& \leq \# \mathbb{Z}^{M}(\Omega) \cdot \mathbb{H}_{\varepsilon}\left(\left.\mathcal{K}\right|_{\left[0, M^{-1} R\right]^{n+1}}\right) \\
& =\operatorname{vol}\left(\Omega_{M}\right) M^{-n+1} \mathbb{H}_{\varepsilon}\left(\left.\mathcal{K}\right|_{\left[0, M^{-1} R\right]^{n+1}}\right) .
\end{aligned}
$$

Dividing (3.20) by $R^{n+1} \operatorname{vol}(\Omega)$ and taking the limit as $R \rightarrow \infty$ we obtain

$$
\frac{1}{\operatorname{vol}(\Omega)} \limsup _{R \rightarrow \infty} \mathbb{H}_{\varepsilon}\left(\left.\mathcal{K}\right|_{R \cdot \Omega}, L^{\infty}(R \cdot \Omega)\right) \leq \frac{\operatorname{vol}\left(\Omega_{M}\right)}{\operatorname{vol}(\Omega)} \overline{\mathbb{H}}_{\varepsilon}\left(\mathcal{K}, L^{\infty}\left(\mathbb{R}^{n+1}\right)\right) .
$$

Since we have assumed that the boundary of $\Omega$ is piecewise smooth, $\operatorname{vol}\left(\Omega_{M}\right)$ tends to $\operatorname{vol}(\Omega)$ as $M \rightarrow \infty$. Therefore (3.21) implies that

$$
\frac{1}{\operatorname{vol}(\Omega)} \limsup _{R \rightarrow \infty} \mathbb{H}_{\mathcal{\varepsilon}}\left(\left.\mathcal{K}\right|_{R \cdot \Omega}, L^{\infty}(R \cdot \Omega)\right) \leq \overline{\mathbb{H}}_{\mathcal{E}}\left(\mathcal{K}, L^{\infty}\left(\mathbb{R}^{n+1}\right)\right) .
$$

To prove the reverse inequality we consider the set $\Omega_{1}:=(0,1)^{n+1} \backslash \bar{\Omega}$. Then $\Omega_{1}$ also satisfies the hypotheses of the theorem and therefore, similarly to (3.22), we have

$$
\frac{1}{\operatorname{vol}\left(\Omega_{1}\right)} \limsup _{R \rightarrow \infty} \mathbb{H}_{\varepsilon}\left(\left.\mathcal{K}\right|_{R \cdot \Omega_{1}}, L^{\infty}\left(R \cdot \Omega_{1}\right)\right) \leq \overline{\mathbb{H}}_{\varepsilon}\left(\mathcal{K}, L^{\infty}\left(\mathbb{R}^{n+1}\right)\right) .
$$

On the other hand, (3.11) implies that

$$
\frac{1}{R^{n+1}} \mathbb{H}_{\mathcal{\varepsilon}}\left(\left.\mathcal{K}\right|_{[0, R]^{n+1}}\right) \leq \frac{\operatorname{vol}(\Omega)}{\operatorname{vol}(R \cdot \Omega)} \mathbb{H}_{\varepsilon}\left(\left.\mathcal{K}\right|_{R \cdot \Omega}\right)+\frac{\operatorname{vol}\left(\Omega_{1}\right)}{\operatorname{vol}\left(R \cdot \Omega_{1}\right)} \mathbb{H}_{\mathcal{E}}\left(\left.\mathcal{K}\right|_{R \cdot \Omega_{1}}\right) .
$$

Since $\operatorname{vol}(\Omega)+\operatorname{vol}\left(\Omega_{1}\right)=1$ and the left-hand side of $(3.24)$ tends to $\overline{\mathbb{H}}_{\varepsilon}\left(\mathcal{K}, L^{\infty}\left(\mathbb{R}^{n+1}\right)\right)$, it follows from (3.22), (3.23), and (3.24) that the limit on the right-hand side of (3.18) exists and coincides with $\overline{\mathbb{H}}_{\varepsilon}\left(\mathcal{K}, L^{\infty}\left(\mathbb{R}^{n+1}\right)\right)$. This proves Theorem 3.1.

Finally, in this section we state a useful corollary of the theorem proved above.

Corollary 3.3. Let $\left(t^{\prime}, h^{\prime}\right):=\mathbb{A}(t, h)$ be an arbitrary linear invertible transformation of space-time $\mathbb{R}^{n+1}$ and let

$$
\mathbb{S}_{(t, h)}^{\prime}:=\mathbb{S}_{\mathbb{A}(t, h)}
$$

Then the topological entropies of the semigroups $\mathbb{S}_{(t, h)}^{\prime}$ and $\mathbb{S}_{(t, h)}$ are connected by the relation

$$
h_{\text {top }}\left(\mathcal{A}, \mathbb{S}_{(t, h)}^{\prime}\right)=\operatorname{det}(\mathbb{A}) h_{\text {top }}\left(\mathcal{A}, \mathbb{S}_{(t, h)}\right) .
$$

Indeed, (3.26) follows immediately from Proposition 3.1, Theorem 3.1, and the fact that $\operatorname{vol}(\mathbb{A} \Omega)=\operatorname{det}(\mathbb{A}) \operatorname{vol}(\Omega)$, which is obvious.

\section{Generalized topological directional entropies}

In this section we introduce several additional dynamic characteristics of the action of the multiparameter semigroup (1.6) on the attractor, connected with some distinguished hyperplane in space-time $\mathbb{R}^{n+1}$, and obtain some relations between them. 
Definition 4.1. Let $V_{k} \subset \mathbb{R}^{n+1}$ be an arbitrary $k$-dimensional hyperplane in $\mathbb{R}^{n+1}$. We define the corresponding $k$-parameter subsemigroup of the extended semigroup (1.6) by the formula

$$
\mathbb{S}_{(t, h)}^{V_{k}}:=\left\{\mathbb{S}_{(t, h)}, t \geq 0,(t, h) \in V_{k}\right\}
$$

Remark 4.1. The most natural examples of the choice of the hyperplane $V_{k}$ are the following.

1. $V_{n+1}:=\mathbb{R}^{n+1}$. Then $\mathbb{S}_{(t, h)}^{V_{n+1}}=\mathbb{S}_{(t, h)}$ and therefore the semigroup (4.1) coincides with (1.6) and describes the space-time dynamics generated by (0.1).

2. $V_{n}:=\mathbb{R}_{x}^{n}$. Then obviously $\mathbb{S}_{(t, h)}^{V_{n}}=T_{h}$, and therefore the semigroup (4.1) describes the spatial structure of the attractor $\mathcal{A}$.

3. $V_{1}:=\mathbb{R}_{t}^{1}$. In this case $\mathbb{S}_{(t, h)}^{V_{1}}=S_{t}$ and therefore the semigroup (4.1) describes the temporal evolution generated by equation (4.1).

Note, however, that by choosing one of the intermediate hyperplanes $V_{k}$, we obtain a description of the interaction between the spatial and temporal modes of the system under consideration (for example, travelling waves). This choice is of interest both from a theoretical standpoint and in view of the applications.

Proceeding in a similar way to the preceding section we can define the topological entropy $h_{\text {top }}\left(\mathcal{A}, \mathbb{S}_{(t, h)}^{V_{k}}\right)$ of the action of the subsemigroups (4.1) on the attractor. However, as we will show in the following sections, for $k<n+1$ these quantities, as a rule, turn out to be infinite even if the topological entropy $h_{\text {top }}\left(\mathcal{A}, \mathbb{S}_{(t, h)}\right)$ is zero. Therefore following [44] we introduce generalized topological entropies $\widehat{h}_{\text {top }}^{n+1-k}\left(\mathcal{A}, \mathbb{S}_{(t, h)}^{V_{k}}\right)$, which turn out to be finite for the case of the dynamics generated by equation $(0.1)$.

Definition 4.2. Fix the metric of the space $L_{e^{-|x|}}^{\infty}\left(\mathbb{R}^{n}\right)$ on the attractor. Let $V_{k}$ be an arbitrary hyperplane in $\mathbb{R}^{n+1}$ and let $\left\{e_{1}, \ldots, e_{k}\right\}$ be an orthonormal basis in $V_{k}$ such that

$$
(t, x):=\sum_{i=1}^{k} \xi_{i} e_{i} \in V_{K}^{+}:=V_{k} \cap\{t \geq 0\} \quad \forall \xi_{i} \geq 0 .
$$

As in Definition 3.1, for any $R>0$ we introduce the following equivalent metric on the attractor:

$$
d_{V_{k}}^{R}\left(u_{1}, u_{2}\right):=\sup _{\xi \in[0, R]^{k}}\left\|\mathbb{S}_{\sum_{i=1}^{k} \xi_{i} e_{i}} u_{1}-\mathbb{S}_{\sum_{i=1}^{k} \xi_{i} e_{i}} u_{2}\right\|_{L_{e^{-}|x|}^{\infty}\left(\mathbb{R}^{n}\right)} .
$$

We define the generalized topological entropy of the semigroup (4.1) to be the number

$$
\widehat{h}_{\mathrm{top}}^{n+1-k}\left(\mathcal{A}, \mathbb{S}_{(t, h)}^{V_{k}}\right):=\limsup _{\varepsilon \rightarrow 0}\left(\ln \frac{1}{\varepsilon}\right)^{k-n-1} \limsup _{R \rightarrow \infty} \frac{1}{R^{k}} \mathbb{H}_{\varepsilon}\left(\mathcal{A}, d_{V_{k}}^{R}\right) \text {. }
$$

Remark 4.2. Standard arguments based on the subadditivity (3.11) (see, for example, Proposition 3.1 and Theorem 3.1) show that the quantity (4.3) depends on the hyperplane $V_{k}$, but not on the choice of an orthonormal basis in it. On the other hand, unlike the classical topological entropy $h_{\text {top }}\left(\mathcal{A}, \mathbb{S}_{(t, h)}\right)$ (or $h_{\text {top }}\left(\mathcal{A}, \mathbb{S}_{(t, h)}^{V_{k}}\right)$ ), the generalized entropies (4.3) are not in general topological invariants, and in particular they depend on how the metric of the local topology on the attractor is defined. In Definition 4.1 we have fixed what seems to us to be the most natural choice of metrization of this topology, using a weight function $\phi(x)=e^{-|x|}$ that decays exponentially. Moreover, as we will show in $\S 10$, for $k<n$ there are no reasonable topological invariants of the action of the semigroup $\mathbb{S}_{(t, h)}^{V_{k}}$ on the attractor. 
The following obvious proposition shows that, similarly to the fractal dimension, the generalized entropies are Lipschitzian invariants.

Proposition 4.1. Suppose that $T:\left(\mathcal{A}, d_{1}\right) \rightarrow\left(\overline{\mathcal{A}}, d_{2}\right)$ is a homeomorphism such that the mappings $T$ and $T^{-1}$ satisfy Hölder's condition with index $\alpha \in(0,1]$. Define an action of the semigroup $\overline{\mathbb{S}}_{(t, h)}$ on $\left(\overline{\mathcal{A}}, d_{2}\right)$ by the formula

$$
\overline{\mathbb{S}}_{(t, h)}:=T \circ \mathbb{S}_{(t, h)} \circ T^{-1}
$$

Then the generalized entropies of these semigroups satisfy the inequalities

$$
\begin{aligned}
\alpha^{n+1-k} \widehat{h}_{\mathrm{top}}^{n+1-k}\left(\left(\overline{\mathcal{A}}, d_{2}\right), \overline{\mathbb{S}}_{(t, h)}^{V_{k}}\right) & \leq \widehat{h}_{\mathrm{top}}^{n+1-k}\left(\left(\mathcal{A}, d_{1}\right), \mathbb{S}_{(t, h)}^{V_{k}}\right) \\
& \leq \alpha^{k-n-1} h_{\mathrm{top}}^{n+1-k}\left(\left(\overline{\mathcal{A}}, d_{2}\right), \overline{\mathbb{S}}_{(t, h)}^{V_{k}}\right),
\end{aligned}
$$

where the symbol $\left(\mathcal{A}, d_{1}\right)$ is used to emphasize the dependence of the generalized entropies on the metric.

To study the generalized entropies (4.3) it is convenient to introduce the following equivalent quantities.

Definition 4.3. Let $\mathcal{K}$ be the set of all bounded solutions of $(0.1)$ defined in Theorem 3.1. Define the action of the $(n+1)$-parameter group $\left\{\mathbb{T}_{(s, h)},(s, h) \in \mathbb{R}^{n+1}\right\}$ by the formula

$$
\left(\mathbb{T}_{(s, h)} u\right)(t, x)=u(t+s, x+h), \quad \mathbb{T}_{(s, h)} \mathcal{K}=\mathcal{K} .
$$

We endow the set $\mathcal{K}$ with the metric

$$
d\left(u_{1}, u_{2}\right):=\left\|u_{1}-u_{2}\right\|_{L_{e^{-|(t, x)|}}^{\infty}\left(\mathbb{R}^{n+1}\right)},
$$

and for any hyperplane $V_{k} \subset \mathbb{R}^{n+1}$ we define the generalized topological entropies $\widehat{h}_{\text {top }}^{n+1-k}\left(\mathcal{K}, \mathbb{T}_{(s, h)}^{V_{k}}\right)$ by analogy with $(4.3)$.

The following proposition is an analogue of Proposition 3.1 and Theorem 3.1 for the case of generalized directional entropies.

Proposition 4.2. Let $V_{k}$ be an arbitrary $k$-dimensional hyperplane in space-time $\mathbb{R}^{n+1}$, let $V_{k}^{\perp}$ be its orthogonal complement, and let $\Omega \subset V_{k}$ be an arbitrary domain in $V_{k}$ with piecewise smooth boundary. Then

$$
\begin{aligned}
\widehat{h}_{\text {top }}^{n+1-k} & \left(\mathcal{K}, \mathbb{T}_{(s, h)}^{V_{k}}\right) \\
& =\limsup _{\varepsilon \rightarrow 0}\left(\ln \frac{1}{\varepsilon}\right)^{k-n-1} \lim _{R \rightarrow \infty} \frac{1}{\operatorname{vol}_{k}(R \cdot \Omega)} \mathbb{H}_{\varepsilon}\left(\left.\mathcal{K}\right|_{R \cdot \Omega \times V_{k}}, L_{\phi_{V_{k}}}^{\infty}\left(\mathbb{R}^{n+1}\right)\right),
\end{aligned}
$$

where $\phi_{V_{k}}(t, x):=e^{-\operatorname{dist}\left((t, x), V_{k}\right)}$ and $\operatorname{vol}_{k}(V)$ is the Lebesgue measure in the hyperplane $V_{k}$.

The proof of (4.6) repeats almost word for word the proof of Proposition 3.1 and Theorem 3.1 and is therefore omitted.

The next proposition shows that the entropies introduced in Definition 4.3 are, in a sense, equivalent to the generalized entropies of $\mathcal{A}$ defined above.

Proposition 4.3. There exists a positive constant $C$ such that for any hyperplane $V_{k}$

$$
C^{-1} \widehat{h}_{\text {top }}^{n+1-k}\left(\mathcal{K}, \mathbb{T}_{(s, h)}^{V_{k}}\right) \leq \widehat{h}_{\text {top }}^{n+1-k}\left(\mathcal{A}, \mathbb{S}_{(t, h)}^{V_{k}}\right) \leq C \widehat{h}_{\text {top }}^{n+1-k}\left(\mathcal{K}, \mathbb{T}_{(s, h)}^{V_{k}}\right) .
$$

Proof. We define the set $\mathcal{K}^{+}:=\left.\mathcal{K}\right|_{t \geq 0} \subset L^{\infty}\left(\mathbb{R}_{+} \times R^{n}\right)$. Then, since the attractor $\mathcal{A}$ is bounded in $\Phi_{b}$, for any $0<\beta_{1} \leq \beta_{2}$ and any $u_{1}, u_{2} \in \mathcal{A}$, we have

$$
\left\|u_{1}-u_{2}\right\|_{\Phi_{e^{-\beta_{1}|x|}}} \leq\left\|u_{1}-u_{2}\right\|_{\Phi_{b}}^{1-\beta_{1} / \beta_{2}} \cdot\left\|u_{1}-u_{2}\right\|_{\Phi_{e}-\beta_{2}|x|}^{\beta_{1} / \beta_{2}} \leq C_{1}\left\|u_{1}-u_{2}\right\|_{\Phi_{e}^{-\beta_{2}|x|}}^{\beta_{1} / \beta_{2}} .
$$


Hence the identity mapping

$$
i:\left(\mathcal{A}, d_{e^{-|x|}}\right) \rightarrow\left(\mathcal{A}, d_{e^{-\alpha|x|}}\right),
$$

where $\alpha>0$ is the same as in Theorem 1.2, is a Hölder homeomorphism. On the other hand, by Theorem 1.2 the solution operator of $(0.1), \mathbb{S}: u_{0} \rightarrow u$, defines the Lipschitz homeomorphism

$$
\mathbb{S}:\left(\mathcal{A}, d_{e^{-\alpha|x|}}\right) \rightarrow\left(\mathcal{K}^{+}, d_{e^{-K t-\alpha|x|}}\right)
$$

and this homeomorphism obviously maps the semigroup $\mathbb{S}_{(t, h)}$ into the semigroup $\left\{\mathbb{T}_{(t, h)}, t \geq 0, h \in \mathbb{R}^{n}\right\}$. Finally, similarly to (4.9), the identity mapping

$$
j:\left(\mathcal{K}^{+}, d_{e^{-L t-\alpha|x|}}\right) \rightarrow\left(\mathcal{K}^{+}, d_{e^{-|(t, x)|}}\right)
$$

is also a Hölder homeomorphism. Thus, it follows from Proposition 4.1 that

$$
C_{2}^{-1} \widehat{h}_{\text {top }}^{n+1-k}\left(\mathcal{K}^{+}, \mathbb{T}_{(s, h)}^{V_{k}}\right) \leq \widehat{h}_{\text {top }}^{n+1-k}\left(\mathcal{A}, \mathbb{S}_{(t, h)}^{V_{k}}\right) \leq C_{2} \widehat{h}_{\text {top }}^{n+1-k}\left(\mathcal{K}^{+}, \mathbb{T}_{(s, h)}^{V_{k}}\right)
$$

for any hyperplane $V_{k}$. Consequently, to complete the proof it is sufficient to show that

$$
2^{k-n-1} \widehat{h}_{\text {top }}^{n+1-k}\left(\mathcal{K}, \mathbb{T}_{(s, h)}^{V_{k}}\right) \leq \widehat{h}_{\text {top }}^{n+1-k}\left(\mathcal{K}^{+}, \mathbb{T}_{(s, h)}^{V_{k}}\right) \leq \widehat{h}_{\text {top }}^{n+1-k}\left(\mathcal{K}, \mathbb{T}_{(s, h)}^{V_{k}}\right) .
$$

Since the right-hand inequality in (4.11) is clear, it is sufficient to verify only the left-hand one. First of all, we observe that $e^{-|(t, x)|} \leq \frac{\varepsilon}{R_{0}}$ if $t \leq-\ln \frac{R_{0}}{\varepsilon}$, where $R_{0}:=\|\mathcal{K}\|_{L^{\infty}\left(\mathbb{R}^{n+1}\right)}$. Hence,

$$
\mathbb{H}_{\varepsilon}\left(\mathcal{K}, d_{V_{k}}^{R}\right) \leq \mathbb{H}_{\varepsilon}\left(T_{-\ln \frac{R_{0}}{\varepsilon}} \mathcal{K}^{+}, d_{V_{k}}^{R}\right),
$$

where $\left(T_{s} u\right)(t, x):=u(t+s, x)$. On the other hand, since $\phi(t, x)$ is a weight function with exponential growth rate 1 (with $C_{\phi}=1$ ) in $\mathbb{R}^{n+1}$, inequality (1.24) implies that $\left(T_{\ln \frac{R_{0}}{\varepsilon}} \phi\right)(t, x) \leq R_{0} \varepsilon^{-1} \phi(t, x)$. Hence,

$$
\mathbb{H}_{\varepsilon}\left(T_{-\ln \frac{1}{\varepsilon}} \mathcal{K}^{+}, d_{V_{k}}^{R}\right) \leq \mathbb{H}_{\frac{\varepsilon^{2}}{R_{0}}}\left(\mathcal{K}^{+}, d_{V_{k}}^{R}\right) .
$$

It follows from (4.12) and (4.13) that

$$
\widehat{h}_{\text {top }}^{n+1-k}\left(\mathcal{K}, \mathbb{T}_{(s, h)}^{V_{k}}\right) \leq 2^{n+1-k} \widehat{h}_{\text {top }}^{n+1-k}\left(\mathcal{K}^{+}, \mathbb{T}_{(s, h)}^{V_{k}}\right) .
$$

Proposition 4.3 is proved.

We are now ready to prove the main result of this section.

Theorem 4.1. Suppose that the hypotheses of Theorem 1.1 hold. Then

(1) for any hyperplane $V_{k} \subset \mathbb{R}^{n+1}$ the corresponding generalized topological entropy is finite:

$$
\widehat{h}_{\mathrm{top}}^{n+1-k}\left(\mathcal{A}, \mathbb{S}_{(t, h)}^{V_{k}}\right) \leq C<\infty,
$$

where the constant $C$ does not depend on the choice of the plane $V_{k}$;

(2) for any two hyperplanes $V_{k}, V_{k^{\prime}} \subset \mathbb{R}^{n+1}$ such that $V_{k} \subset V_{k^{\prime}}$

$$
\widehat{h}_{\text {top }}^{n+1-k^{\prime}}\left(\mathcal{A}, \mathbb{S}_{(t, h)}^{V_{k^{\prime}}}\right) \leq L^{k^{\prime}-k} \widehat{h}_{\mathrm{top}}^{n+1-k}\left(\mathcal{A}, \mathbb{S}_{(t, h)}^{V_{k}}\right),
$$

where the constant $L$ is independent of the hyperplanes $V_{k}$ and $V_{k^{\prime}}$.

Proof. By Proposition 4.3 it is sufficient to verify (4.14) and (4.15) for the entropies $\widehat{h}_{\text {top }}^{V_{k}}\left(\mathcal{K}, \mathbb{T}_{(s, h)}^{V_{k}}\right)$. First we prove (4.14). For the domain $\Omega$ in (4.6) we take the the unit cube $\mathcal{C}_{V_{k}}$ generated by some orthonormal basis $\left\{e_{1}, \ldots, e_{k}\right\}$ in $V_{k}$. Then

$$
\mathbb{H}_{\varepsilon}\left(\left.\mathcal{K}\right|_{R \cdot \mathcal{C}_{V_{k}} \times V_{k}^{\perp}}, L_{\phi_{V_{k}}}^{\infty}\left(\mathbb{R}^{n+1}\right)\right) \leq \mathbb{H}_{\varepsilon}\left(\left.\mathcal{K}\right|_{\mathcal{O}_{\ln \frac{R_{0}}{\varepsilon}}\left(R \cdot \mathcal{C}_{V_{k}}\right)}, L^{\infty}\left(\mathbb{R}^{n+1}\right)\right),
$$


where the symbol $\mathcal{O}_{\delta}(M)$ denotes the $\delta$-neighborhood of a set $M$ in $\mathbb{R}^{n+1}$. Now it follows from (3.11) and (2.25) that

$$
\begin{aligned}
\mathbb{H}_{\varepsilon}\left(\left.\mathcal{K}\right|_{\mathcal{O}_{\ln \frac{R_{0}}{\varepsilon}}\left(R \cdot \mathcal{C}_{V_{k}}\right)}\right. & \leq\left(\frac{R}{\ln \frac{R_{0}}{\varepsilon}}+1\right)^{k} \mathbb{H}_{\varepsilon}\left(\left.\mathcal{K}\right|_{\left[0, \sqrt{n+1} \ln \frac{R_{0}}{\varepsilon}\right]^{n+1}}\right) \\
& \leq C\left(\frac{R}{\ln \frac{R_{0}}{\varepsilon}}+1\right)^{k}\left(\ln \frac{1}{\varepsilon}\right)^{n+1} \\
& =C R^{k}\left(\ln \frac{1}{\varepsilon}\right)^{n+1-k}+C\left(R^{k-1}+1\right)\left(\ln \frac{1}{\varepsilon}\right)^{n+1} .
\end{aligned}
$$

By substituting (4.16) and (4.17) into the right-hand side of (4.6) we see that the entropy $\widehat{h}_{\text {top }}^{n+1-k}\left(\mathcal{K}, \mathbb{T}_{(s, h)}^{V_{k}}\right)$ satisfies $(4.14)$ as required.

We now prove estimate $(4.15)$. It is obviously sufficient to prove it only for the case $k^{\prime}=k+1$. We fix an orthonormal basis $\left\{e_{1}, \ldots, e_{k+1}\right\}$ in $V_{k+1}$ so that the vectors $\left\{e_{1}, \ldots, e_{k}\right\}$ form an orthonormal basis in $V_{k}$. Then, clearly, $\mathcal{C}_{V_{k+1}}=\mathcal{C}_{V_{k}} \times[0,1]$. Note also that using the triangle inequality and the definition of the weight functions $\phi_{V_{k}}$ and $\phi_{V_{k+1}}$ we obtain the estimate

$$
\phi_{V_{k+1}}(t, x) \leq \varepsilon^{-1} \phi_{V_{k}}(t, x) \quad \text { if } \quad \operatorname{dist}\left(\Pi_{V_{k+1}}(t, x), V_{k}\right) \leq \ln \frac{1}{\varepsilon},
$$

where the symbol $\Pi_{V_{k+1}}$ denotes the orthogonal projection onto the hyperplane $V_{k+1}$. Hence,

$$
\mathbb{H}_{\varepsilon}\left(\left.\mathcal{K}\right|_{\left(R \cdot \mathcal{C}_{V_{k}} \times\left[0, \ln \frac{1}{\varepsilon}\right]\right) \times V_{k+1}^{\perp}}, L_{\phi_{V_{k+1}}}^{\infty}\right) \leq \mathbb{H}_{\varepsilon^{2}}\left(\left.\mathcal{K}\right|_{R \cdot \mathcal{C}_{V_{k}} \times V_{k}^{\perp}}, L_{\phi_{V_{k}}}^{\infty}\right) .
$$

From (4.19) and the subadditivity (3.11) it follows immediately that for sufficiently large $R$

$$
\mathbb{H}_{\varepsilon}\left(\left.\mathcal{K}\right|_{R \cdot \mathcal{C}_{V_{k+1}} \times V_{k+1}^{\perp}}, L_{\phi_{V_{k+1}}}^{\infty}\right) \leq\left(\frac{R}{\ln \frac{1}{\varepsilon}}+1\right) \mathbb{H}_{\varepsilon^{2}}\left(\left.\mathcal{K}\right|_{R \cdot \mathcal{C}_{V_{k}} \times V_{k}^{\perp}}, L_{\phi_{V_{k}}}^{\infty}\right)
$$

Multiplying inequality $(4.20)$ by $R^{-k-1}\left(\ln \frac{1}{\varepsilon}\right)^{n-k}$ and passing to the limit (first as $R \rightarrow$ $\infty$, and then as $\varepsilon \rightarrow 0$ ) we obtain

$$
\widehat{h}_{\text {top }}^{n-k}\left(\mathcal{K}, \mathbb{T}_{(s, h)}^{V_{k+1}}\right) \leq 2^{n+1-k} \widehat{h}_{\text {top }}^{n+1-k}\left(\mathcal{K}, \mathbb{T}_{(s, h)}^{V_{k}}\right)
$$

This completes the proof of Theorem 4.1 .

Remark 4.3. Suppose that the classical topological entropy of the semigroup (1.6) is strictly positive, that is, $h_{\text {top }}\left(\mathcal{A}, \mathbb{S}_{(t, h)}\right)=\widehat{h}_{\text {top }}^{0}\left(\mathcal{A}, \mathbb{S}_{(t, h)}^{\mathbb{R}^{n+1}}\right)>0$, for some equation of the form (0.1); examples of such equations were constructed in 33. Then it follows from the second part of Theorem 4.1 that the generalized entropy corresponding to an arbitrary hyperplane $V_{k} \subset \mathbb{R}^{n+1}$ is also strictly positive. On the other hand, suppose that the zero-dimensional entropy (corresponding to the 0-dimensional hyperplane)

$$
\widehat{h}_{\text {top }}^{n+1}\left(\mathcal{A}, \mathbb{S}_{(t, h)}^{\{0\}}\right)=\limsup _{\varepsilon \rightarrow 0}\left(\ln \frac{1}{\varepsilon}\right)^{-n-1} \mathbb{H}_{\varepsilon}\left(\mathcal{A}, L_{e^{-|x|}}^{\infty}\left(\mathbb{R}^{n}\right)\right)
$$

is equal to zero. Then all the generalized topological entropies are also zero.

Moreover, (4.15) can be interpreted as a generalization of the inequality

$$
h_{\text {top }}(\mathcal{A}) \leq L \operatorname{dim}_{F}(\mathcal{A})
$$

between the fractal dimension of an invariant set and the topological entropy for classical dynamical systems. Indeed, the case of ordinary differential equations of the form (0.1) 
corresponds to $n=0$. Therefore it is easy to see that (4.21) coincides with the fractal dimension of the attractor, while the choice of the "hyperplane" $V_{1}=R_{t}$ gives the topological entropy of this system. In this case (4.15) takes the classical form (4.22). Extending this analogy we can interpret (4.21) as the generalized fractal dimension of the attractor $\mathcal{A}$.

Remark 4.4. An analogue of Proposition 4.2 can also be formulated directly for the quantity $\widehat{h}_{\text {top }}^{n+1-k}\left(\mathcal{A}, \mathbb{S}_{(t, h)}^{V_{k}}\right)$; see [44. However, by contrast with the entropies calculated with respect to $\mathcal{K}$, in the formula for calculating the generalized entropies of the attractor $\mathcal{A}$, the space and time variables ( $x, t$, respectively) are involved in a nonsymmetric way. This leads to more cumbersome formulae and makes studying them more difficult. Moreover, the possibility of "interchanging" space and time variables will play an essential role in our study of space-time chaos (see $\S 10$ ).

\section{INJECTIVITY OF THE SOLUTION OPERATOR AND THE TEMPORAL EVOLUTION OF SPACE-CHAOTIC STRUCTURES}

In this section we will show that the semigroup $\left\{S_{t}, t \geq 0\right\}$ generated by $(0.1)$, when restricted to the attractor $\mathcal{A}$, can be extended to a group of Hölder homeomorphisms of the attractor with Hölder index arbitrarily close to 1 . We will apply this result to investigate the temporal evolution of the generalized topological entropies in spatial directions of some subsets of the attractor. (These are obviously invariant under spatial translations, but are not invariant under $S_{t}$.) We begin with the following theorem.

Theorem 5.1. Suppose that the hypotheses of Theorem 1.1 hold. Then for any $t \geq 0$ the solution operator $S_{t}$ of (0.1) is invertible on the attractor. Moreover, for any $\delta>0$ and any $t \geq 0$ there exists $\alpha=\alpha(t, \delta)>0$ such that the inverse operator $S_{-t}:=\left(S_{t}\right)^{-1}$ satisfies

$$
\left\|S_{-t} u_{1}-S_{-t} u_{2}\right\|_{L_{e^{-\alpha|x|}}^{\infty}\left(\mathbb{R}^{n}\right)} \leq C\left\|u_{1}-u_{2}\right\|_{L_{e^{-\alpha|x| / 2}}^{\infty-\delta}}^{\left.1-\mathbb{R}^{n}\right)}, \quad u_{1}, u_{2} \in \mathcal{A},
$$

where the constant $C=C(t, \delta)$ is independent of $u_{1}, u_{2} \in \mathcal{A}$.

As usual (see [2, 43, 44]), the proof of (5.1) is based on verifying a "logarithmic convexity" property for solutions of the corresponding linearized equation. To do this we need the following abstract result proved in [13].

Lemma 5.1 (13]). Let $H$ be a Hilbert space, $B: D(B) \rightarrow H$ a linear (unbounded) operator in it, and let $v \in C^{1}\left(\left[t_{0}, t_{1}\right], H\right) \cap C\left(\left[t_{0}, t_{1}\right], D(B)\right)$ be a solution of the equation

$$
\partial_{t} v-B v=P(t) v, \quad\|P(t)\|_{H \rightarrow H} \leq P_{0} .
$$

Suppose also that $B=B_{+}+B_{-}^{\prime}+B_{-}^{\prime \prime}$, where $B_{+}$is a symmetric operator, and $B_{-}^{\prime}$ and $B_{-}^{\prime \prime}$ are antisymmetric operators such that for any $w \in H$

$$
\begin{gathered}
\left(B_{+} w, B_{-}^{\prime} w\right)_{H} \geq-\gamma\left\|B_{+} w\right\|_{H}\|w\|_{H}-\beta\|w\|_{H}^{2}, \\
\left\|B_{-}^{\prime \prime} w\right\|_{H}^{2} \leq \gamma\left\|B_{+} w\right\|_{H}\|w\|_{H}+\beta\|w\|_{H}^{2} .
\end{gathered}
$$

Define the function

$$
l(t):=2 \ln \|u(t)\|_{H}-\int_{t_{0}}^{t} \psi(s) d s, \quad \psi(t):=2 \frac{(P(t) u(t), u(t))}{\|u(t)\|_{H}^{2}} .
$$

Then for any $t_{0} \leq t \leq t_{1}$

$$
l(t) \leq \alpha_{ \pm} l\left(t_{0}\right)+\left(1-\alpha_{ \pm}\right) l\left(t_{1}\right)+e^{4 \gamma\left(t_{1}-t_{0}\right)}\left(t_{1}-t_{0}\right)^{2}\left(8 \gamma^{2}+4 \beta+2 P_{0}^{2}\right),
$$


with

$$
\alpha_{ \pm}:=\frac{e^{ \pm 4 \gamma t_{1}}-e^{ \pm 4 \gamma t}}{e^{ \pm 4 \gamma t_{1}}-e^{ \pm 4 \gamma t_{0}}},
$$

where the minus sign is chosen if $l\left(t_{0}\right) \leq l\left(t_{1}\right)$, and the plus sign if $l\left(t_{0}\right) \geq l\left(t_{1}\right)$.

Lemma 5.2. Suppose that the assumptions of Lemma 5.1 hold and suppose that the solution $v(t)$ is known to be defined on $\left(-\infty, t_{1}\right]$ and bounded, $\|v(t)\|_{H} \leq K$. Then for any $\mu>0$ and $t \in\left(-\infty, t_{1}\right)$ there exists a constant $C=C\left(t, t_{1}, \mu, K\right)$ such that

$$
\|u(t)\|_{H} \leq C\left\|u\left(t_{1}\right)\right\|_{H}^{1-\delta}, \quad 1-\delta:=e^{4 \gamma\left(t-t_{1}\right)}-\mu .
$$

Proof. Taking the exponential of (5.6) and using the inequality

$$
-2 P_{0}\left(t-t_{0}\right) \leq \int_{t_{0}}^{t} \psi(s) d s \leq 2 P_{0}\left(t-t_{0}\right),
$$

which is obvious, we obtain

$$
\|u(t)\|_{H} \leq C\left(t, t_{1}, t_{0}\right)\left\|u\left(t_{1}\right)\right\|_{H}^{1-\alpha_{ \pm}}\left\|u\left(t_{0}\right)\right\|_{H}^{\alpha_{ \pm}} .
$$

Since $\left\|u\left(t_{0}\right)\right\|_{H} \leq K$, from (5.9) we get

$$
\|u(t)\|_{H} \leq C^{\prime}\left(K, t, t_{0}, t_{1}\right)\left\|u\left(t_{1}\right)\right\|_{H}^{1-\delta},
$$

where $\delta=\max \left\{\alpha_{+}, \alpha_{-}\right\}$. We now fix $t_{2}=-N$, where $N>0$ is sufficiently large. Then

$$
1-\delta=1-\alpha_{+}=\frac{e^{4 \gamma t}-e^{-4 \gamma N}}{e^{4 \gamma t_{1}}-e^{-4 \gamma N}} \rightarrow e^{-4 \gamma\left(t_{1}-t\right)},
$$

as $N \rightarrow \infty$. Hence for any $\mu>0$ there exists $N=N(\mu)$ such that $1-\delta \geq e^{-4 \gamma\left(t_{1}-t\right)}-\mu$. Lemma 5.2 is proved.

Proof of the theorem. Let $u_{1}(t)$ and $u_{2}(t)$ be two solutions of equation (0.1) lying on the attractor. In a similar way to the proof of Theorem 1.2 we define the function $w(t):=\phi_{\alpha}(x)\left(u_{1}(t)-u_{2}(t)\right)$, where the function $\phi_{\alpha}(x)=\phi_{\alpha, 0}(x)$ is defined by (1.10) and $\alpha>0$ is sufficiently small. Then this function satisfies the equation

$$
\partial_{t} w-a \Delta_{x} w+\left(\vec{L}, \nabla_{x}\right) w+\lambda_{0} w+l(t) w=L_{1}(x) w+L_{2}(x) \nabla_{x} w,
$$

where the operators $L_{i}(x), i=1,2$, are calculated using (1.12) and

$$
l(t):=\int_{0}^{1} f^{\prime}\left(s u_{1}(t)+(1-s) u_{2}(t)\right) d s .
$$

We claim that equation (5.12) has the form (5.2). We set $H=L^{2}\left(\mathbb{R}^{n}\right)$,

$$
B_{+}:=a \Delta_{x}-\lambda_{0}, \quad B_{-}^{\prime}:=-\left(\vec{L}, \nabla_{x}\right), \quad B_{-}^{\prime \prime}:=\frac{1}{2}\left(L_{2}(x) \nabla_{x}-\left(L_{2}(x) \nabla_{x}\right)^{*}\right),
$$

where $(L)^{*}$ is the formal conjugate operator of $L$ in the metric of $H$, and

$$
P(t):=L_{1}(x)-l(t)+\frac{1}{2}\left(L_{2}(x) \nabla_{x}+\left(L_{2}(x) \nabla_{x}\right)^{*}\right) .
$$

Then, since the symmetric part of the operator $L_{2}(x) \nabla_{x}$ is obviously an operator of order zero, the operator $P(t)$ we have defined satisfies condition (5.2) with the constant $P_{0}$ independent of the choice of the solutions $u_{1}, u_{2} \in \mathcal{K}$. Moreover, elementary integration by parts shows that

$$
\left(B_{+} v, B_{-}^{\prime} v\right)=0 \quad \forall v \in D(B):=W^{2,2}\left(\mathbb{R}^{n}\right) .
$$


Hence (5.3) is also satisfied. We now verify (5.4). Using (1.13) and (1.14), the interpolation inequality and the regularity of solutions of the Laplace equation in $\mathbb{R}^{n}$ we obtain

$$
\left\|B_{-}^{\prime \prime} v\right\|_{L^{2}\left(\mathbb{R}^{n}\right)} \leq C_{1} \alpha\|v\|_{W^{1,2}\left(\mathbb{R}^{n}\right)}^{2} \leq C_{2} \alpha\|v\|_{H}\|v\|_{D(B)} \leq C_{3} \alpha\|v\|_{H}\left\|B_{+} v\right\|_{H}
$$

for some constants $C_{i}, i=1,2,3$, that are independent of $\alpha$. Thus, equation (5.10) satisfies the hypotheses of Lemma 5.1 with the constant $\gamma:=C_{3} \alpha$, where $C_{3}$ does not depend on $\alpha$. Moreover, it is clear that

$$
\|w(t)\|_{L^{2}\left(\mathbb{R}^{n}\right)} \leq C_{\alpha}\left\|u_{1}(t)-u_{2}(t)\right\|_{L_{e^{-\alpha|x| / 2}}^{\infty}\left(\mathbb{R}^{n}\right)},
$$

so, since the set $\mathcal{K}$ in $L^{\infty}\left(\mathbb{R}^{n+1}\right)$ is bounded, the hypotheses of Lemma 5.2 also hold (with the constant $K$ independent of $u_{1}$ and $u_{2}$ ). Consequently, by Lemma 5.2 we have

$$
\|w(-t-1)\|_{L^{2}\left(\mathbb{R}^{n}\right)} \leq C_{\alpha, t}\left\|u_{1}(0)-u_{2}(0)\right\|_{L_{e^{-\alpha|x| / 2}}^{\infty-\delta}\left(\mathbb{R}^{n}\right)}^{1-},
$$

where the constant $\delta=\delta(t, \alpha)>0$ can be made arbitrarily small by decreasing the constant $\alpha$. To complete the proof it is sufficient to observe that the standard interior estimate applied to the parabolic equation (5.12) gives

$$
\|w(-t)\|_{L^{\infty}\left(\mathbb{R}^{n}\right)} \leq C\|w(-t-1)\|_{L^{2}\left(\mathbb{R}^{n}\right)}
$$

where $C$ is independent of $u_{1}$ and $u_{2}$ (see, for example, [5]). Substituting (5.15) into the right-hand side of (5.16) we obtain (5.1). This completes the proof.

We are now ready to move on to study the temporal evolution of the spatial complexity of solutions of equation (0.1) that lie on the attractor. For this we need the following definition, which is similar to Definitions 4.2 and 3.1 .

Definition 5.1. Let $\mathcal{M} \subset \mathcal{A}$ be an arbitrary closed spatially invariant subset of the attractor (that is, $T_{h} \mathcal{M}=\mathcal{M}$ for any $h \in \mathbb{R}^{n}$ ). Let $\phi=\phi(x)$ be an arbitrary weight function satisfying (2.2). For any $R>0$ we define the following equivalent metric on the attractor:

$$
d_{\phi}^{R}\left(u_{1}, u_{2}\right):=\sup _{h \in[0, R]^{n}}\left\|T_{h} u_{1}-T_{h} u_{2}\right\|_{L_{\phi}^{\infty}\left(\mathbb{R}^{n}\right)} .
$$

By definition, the spatial topological entropy of the set $\mathcal{M}$ is the quantity

$$
\widehat{h}_{s p}\left(\mathcal{M}, T_{h}\right):=\limsup _{\varepsilon \rightarrow 0}\left(\ln \frac{1}{\varepsilon}\right)^{-1} \limsup _{R \rightarrow \infty} \frac{1}{R^{n}} \mathbb{H}_{\varepsilon}\left(\mathcal{M}, d_{\phi}^{R}\right) .
$$

Now let $u_{0} \in \mathcal{A}$ be an arbitrary point on the attractor. We define the spatial topological entropy $\widehat{h}_{s p}\left(u_{0}\right)$ by the following natural formula:

$$
\widehat{h}_{s p}\left(u_{0}\right):=\widehat{h}_{s p}\left(\mathcal{H}_{s p}\left(u_{0}\right), T_{h}\right), \quad \mathcal{H}_{s p}\left(u_{0}\right):=\left[T_{h} u_{0}, h \in \mathbb{R}^{n}\right]_{\Phi_{\text {loc }}} .
$$

The following proposition is an analogue of Proposition 3.1 and Theorem 3.2.

Proposition 5.1. The quantity (5.18) does not depend on the choice of the weight function $\phi$ and can be calculated by the formula

$$
\widehat{h}_{s p}\left(\mathcal{M}, T_{h}\right)=\limsup _{\varepsilon \rightarrow 0}\left(\ln \frac{1}{\varepsilon}\right)^{-1} \lim _{R \rightarrow \infty} \frac{1}{\operatorname{vol}_{n}(R \cdot \Omega)} \mathbb{H}_{\varepsilon}\left(\left.\mathcal{A}\right|_{R \cdot \Omega}, L^{\infty}(R \cdot \Omega)\right),
$$

where $\Omega$ is an arbitrary bounded domain in $\mathbb{R}^{n}$ with piecewise smooth boundary.

The proof of (5.20) repeats word for word the proofs of Proposition 3.1 and Theorem 3.2 and is therefore omitted. 
Remark 5.1. Choosing $\mathcal{M}=\mathcal{A}$ and the weight function $\phi(x):=e^{-|x|}$ we obtain the obvious equality

$$
\widehat{h}_{s p}\left(\mathcal{A}, T_{h}\right)=\widehat{h}_{\text {top }}^{1}\left(\mathcal{A}, \mathbb{S}_{(t, h)}^{\mathbb{R}_{x}^{n}}\right)
$$

(see Definition 4.2). In particular, according to Theorem 4.2 this implies that $\widehat{h}_{s p}\left(\mathcal{M}, T_{h}\right)$ is finite for any $\mathcal{M} \subset \mathcal{A}$. However, as Proposition 5.1 shows, in the special case of the spatial hyperplane $V_{n}:=\mathbb{R}_{x}^{n}$ the generalized entropy $\widehat{h}_{\text {top }}^{1}\left(\mathcal{A}, T_{h}\right)$ does not depend on the choice of the weight function $\phi$. To emphasize this important fact we have repeated the definition of the generalized entropy along a hyperplane, in the special case of the spatial hyperplane $V_{n}:=\mathbb{R}^{n}$ (see Definition 5.1).

Remark 5.2. It is worth noting that, unlike the classical topological entropy $h_{\text {top }}\left(\mathcal{A}, T_{h}\right)$, the quantity $\widehat{h}_{s p}\left(\mathcal{A}, T_{h}\right)$ (or $\left.\widehat{h}_{s p}\left(u_{0}\right)\right)$ is not in general a topological invariant, although it is independent of the choice of the weight function $\phi$. However, as usual, there is the possibility (see, for example, [31]) of constructing a "genuine" topological invariant based on the quantity $\widehat{h}_{s p}\left(\mathcal{A}, T_{h}\right)=\widehat{h}_{s p}\left(\left(\mathcal{A}, d_{\phi}\right), T_{h}\right)$, namely,

$$
\operatorname{dim}_{s p}\left(\mathcal{A}, T_{h}\right):=\inf _{d} \widehat{h}_{s p}\left((\mathcal{A}, d), T_{h}\right),
$$

where the infimum is taken over all possible metrics defining the local topology in $\mathcal{A}$. In the following sections we show that under certain natural conditions on equation (0.1) there exist points $u_{0} \in \mathcal{A}$ such that $\widehat{h}_{s p}\left(u_{0}\right)$ (and even $\operatorname{dim}_{s p}\left(u_{0}\right)$ ) turn out to be strictly positive.

To end this section we show that the quantities introduced in Definition 5.1 do not change along the trajectories of the dynamical system (1.4).

Corollary 5.1. Let $\mathcal{M} \subset \mathcal{A}$ be an arbitrary spatially invariant subset of the attractor. Then for any $t \in \mathbb{R}$ we have

$$
\widehat{h}_{s p}\left(S_{t} \mathcal{M}, T_{h}\right)=\widehat{h}_{s p}\left(\mathcal{M}, T_{h}\right), \quad \text { in particular, } \widehat{h}_{s p}\left(S_{t} u_{0}\right)=\widehat{h}_{s p}\left(u_{0}\right),
$$

for any $u_{0} \in \mathcal{A}$.

In fact, by Theorems 1.2 and 5.1 the operator $S_{t}$ is a Hölder homeomorphism with Hölder index arbitrarily close to 1 , for an appropriate choice of the weighted metrics in $\mathcal{M}$ and $S_{t} \mathcal{M}$, and the entropies (5.18) and (5.19) are independent of the choice of these metrics. Thus (5.23) follows immediately from Proposition 4.1.

\section{Strongly unstable MANifolds of NONHYPERBOliC EQUilibria}

Our next aim is to obtain lower bounds for the generalized entropies introduced in the preceding sections. In this section we present an abstract construction for the structure of a strongly unstable invariant manifold of a smooth mapping in a neighborhood of a nonhyperbolic equilibrium. We will use it in what follows (see $\S \S 7,10)$ to study the attractors of parabolic equations of the form (0.1).

Let $\Phi$ be a Banach space and let $S: \Phi \rightarrow \Phi$ be a nonlinear mapping of the form

$$
\begin{aligned}
S(u) & =S_{0} u+\mathcal{B}(u), \\
S_{0} \in \mathcal{L}(\Phi, \Phi), \quad \mathcal{B} & \in C^{1, \alpha}(\Phi, \Phi), \quad \mathcal{B}(0)=\mathcal{B}^{\prime}(0)=0,
\end{aligned}
$$

where $0<\alpha \leq 1$ is some positive number and $S_{0}:=S^{\prime}(0)$ is the Fréchet derivative of $S$ at zero. Suppose that the zero equilibrium of the mapping $S$ is exponentially unstable, that is,

$$
r_{0}\left(S_{0}\right):=\sup \left|\sigma\left(S_{0}, \Phi\right)\right|>1
$$


where $\sigma\left(S_{0}, \Phi\right)$ denotes the spectrum of the linear operator $S_{0}$ in $\Phi$, and $r_{0}\left(S_{0}\right)$ its spectral radius. To construct an unstable manifold of $S$ we solve the difference equation

$$
u_{n}=S\left(u_{n-1}\right)=S_{0} u_{n-1}+\mathcal{B}\left(u_{n-1}\right), \quad n \in \mathbb{Z}_{-}:=\{0,-1, \ldots\}
$$

in the space of sequences $\left\{u_{n}\right\}_{n=0}^{-\infty}$ with values in the Banach space $\Phi$. To do this we need the following spaces.

Definition 6.1. Let $\gamma>0$ be an arbitrary positive number. The space of $\Phi$-valued sequences $\mathbb{L}_{\gamma}=\mathbb{L}_{\gamma}\left(\mathbb{Z}_{-}, \Phi\right)$ is defined as follows:

$$
\mathbb{L}_{\gamma}:=\left\{u=\left\{u_{n}\right\}_{n=0}^{-\infty}, u_{n} \in \Phi,\|u\|_{\mathbb{L}_{\gamma}}:=\sup _{n \in \mathbb{Z}_{-}}\left(\gamma^{-n}\left\|u_{n}\right\|_{\Phi}\right)<\infty\right\} .
$$

The main result of this section is the following theorem.

Theorem 6.1. Suppose that conditions (6.1) and (6.2) are satisfied. Let $\Phi_{+} \subset \Phi$ be a closed invariant subspace of the linear operator $S_{0}$ satisfying the condition

$$
\inf \left|\sigma\left(\left.S_{0}\right|_{\Phi_{+}}, \Phi_{+}\right)\right|>\theta_{0}>1
$$

where $\theta_{0}$ satisfies the inequality

$$
\theta_{0}^{1+\alpha}>r_{0}\left(S_{0}\right)
$$

Then there exists a ball $B\left(\rho, 0, \Phi_{+}\right)$of radius $\rho>0$ centered at the origin in the space $\Phi_{+}$and a smooth $\left(C^{1+\alpha}\right.$-smooth $)$ mapping

$$
\mathbb{V}: B\left(\rho, 0, \Phi_{+}\right) \rightarrow \mathbb{L}_{\theta_{0}}(\Phi)
$$

such that for any $v_{+} \in \Phi_{+}$the sequence $\mathbb{V}_{n}\left(v_{+}\right):=\mathbb{V}\left(v_{+}\right)(n), n \in \mathbb{Z}_{-}$, satisfies the difference equation (6.3) and

$$
\left\|\mathbb{V}_{n}\left(v_{+}\right)-\left(\left.S_{0}\right|_{\Phi_{+}}\right)^{n} v_{+}\right\|_{\Phi} \leq C \theta_{0}^{(1+\alpha) n}\left\|v_{+}\right\|_{\Phi}^{1+\alpha}
$$

where the constant $C$ is independent of $v_{+} \in B\left(\rho, 0, \Phi_{+}\right)$and $n \in \mathbb{Z}_{-}$.

Proof. First we observe that (6.5) implies that $S_{+}:=\left.S_{0}\right|_{\Phi_{+}}$is invertible and

$$
r_{0}\left(S_{+}^{-1}\right)<\theta_{0}^{-1}
$$

Hence the expression $S_{+}^{n} v_{+}$on the left-hand side of formula (6.8) makes sense. Moreover, it follows from (6.9) and the standard formula for the spectral radius that the sequence $\mathcal{S}_{+} v_{+}:=\left\{S_{+}^{n} v_{+}\right\}_{n=0}^{-\infty}$ is contained in the space $\mathbb{L}_{\theta_{0}}$ and

$$
\left\|\mathcal{S}_{+} v_{+}\right\|_{\mathbb{L}_{\theta_{0}}} \leq C_{1}\left\|v_{+}\right\|_{\Phi} .
$$

We will apply the implicit function theorem to (6.3). To do this we need the following standard result on the solvability of a linear inhomogeneous equation of the form (6.3).

Lemma 6.1. Suppose that the hypotheses of Theorem 6.1 hold and $\lambda_{0}>r_{0}\left(S_{0}\right)$. Then there exists a continuous linear operator

$$
\mathbb{T}_{\lambda_{0}}: \mathbb{L}_{\lambda_{0}} \rightarrow \mathbb{L}_{\lambda_{0}},
$$

such that for any sequence $h=\left\{h_{n}\right\}_{n=0}^{-\infty} \in \mathbb{L}_{\lambda_{0}}$, the sequence $\left\{v_{n}\right\}_{n=0}^{-\infty}:=\mathbb{T}_{\lambda_{0}} h$ satisfies the relation

$$
v_{n}=S_{0} v_{n-1}+h_{n}, \quad n \in \mathbb{Z}_{-} .
$$


Proof. The required linear operator is given by the formula

$$
\left(\mathbb{T}_{\lambda_{0}} h\right)_{n}:=\sum_{k=-\infty}^{n} S_{0}^{n-k} h_{k} .
$$

The convergence of the series on the right-hand side of (6.13) and the estimate

$$
\left\|\mathbb{T}_{\lambda_{0}} h\right\|_{\mathbb{L}_{\lambda_{0}}} \leq C\|h\|_{\mathbb{L}_{\lambda_{0}}}
$$

immediately follow from the condition $\lambda_{0}>r_{0}\left(S_{0}\right)$ and the standard formula for the spectral radius. The fact that the operator (6.14) defines a solution of equation (6.12) can easily be verified by substitution. This proves Lemma 6.1 .

We now define an action of the nonlinear operator $\overline{\mathcal{B}}$ on the space of sequences by the formula

$$
\overline{\mathcal{B}}(u)_{n}:=\mathcal{B}\left(u_{n}\right), \quad u_{n} \in \mathbb{L}_{1}(\Phi),
$$

where the operator $\mathcal{B}$ is defined in (6.1).

Lemma 6.2. Let $\lambda_{0} \geq 1$ be some number. Then the operator $\overline{\mathcal{B}}$ belongs to the class $C^{1, \alpha}\left(\mathbb{L}_{\lambda_{0}}, \mathbb{L}_{\lambda_{0}^{1+\alpha}}\right)$ and

$$
\|\overline{\mathcal{B}}(u)\|_{\mathbb{L}_{\lambda_{0}^{1+\alpha}}} \leq C\|u\|_{\mathbb{L}_{\lambda_{0}}}^{1+\alpha}
$$

and

$$
\left\|\overline{\mathcal{B}}(u)-\overline{\mathcal{B}}\left(u_{1}\right)-\overline{\mathcal{B}}^{\prime}(u)\left(u-u_{1}\right)\right\|_{\mathbb{L}_{\lambda_{0}^{1+\alpha}}} \leq C\left\|u-u_{1}\right\|_{\mathbb{L}_{\lambda_{0}}}^{1+\alpha},
$$

where $u_{1}, u_{2} \in \mathbb{L}_{\lambda_{0}}$ and the constant $C$ depends, generally speaking, on the norms of $u_{1}$ and $u_{2}$.

Proof. Since $\mathcal{B} \in C^{1, \alpha}(\Phi, \Phi)$, we have

$$
\left\|\mathcal{B}\left(u_{n}\right)-\mathcal{B}\left(u_{n}^{1}\right)-\mathcal{B}^{\prime}\left(u_{n}\right)\left(u_{n}-u_{n}^{1}\right)\right\|_{\Phi} \leq C\left\|u_{n}-u_{n}^{1}\right\|_{\Phi}^{1+\alpha}
$$

for some constant $C=C\left(\|u\|_{\mathbb{L}_{1}},\left\|u_{1}\right\|_{\mathbb{L}_{1}}\right)$. Multiplying (6.18) by $\lambda_{0}^{-(1+\alpha) n}$, taking the supremum over $n$ of both parts of this inequality, and using the obvious embedding $\mathbb{L}_{\lambda_{0}} \subset \mathbb{L}_{1}$ (since $\lambda_{0} \geq 1$ ) we obtain (6.17). To prove (6.16) we recall that $\mathcal{B}(0)=\mathcal{B}^{\prime}(0)=0$ and therefore

$$
\left\|\mathcal{B}\left(u_{n}\right)\right\|_{\Phi} \leq C\left(\|u\|_{\mathbb{L}_{1}}\right)\left\|u_{n}\right\|_{\Phi}^{1+\alpha} .
$$

Multiplying this inequality by $\lambda_{0}^{1+\alpha}$ and taking the supremum over $n$ we obtain (6.16). The fact that $\overline{\mathcal{B}}$ is smooth, that is, $\overline{\mathcal{B}} \in C^{1, \alpha}\left(\mathbb{L}_{\lambda_{0}}, \mathbb{L}_{\lambda_{0}^{1+\alpha}}\right)$, is a standard consequence of (6.16) and (6.17). Lemma 6.2 is proved.

We are now ready to complete the proof of the theorem. We first take the inverse of the linear part of equation (6.3) and rewrite it in the form

$$
\mathcal{F}\left(v_{+}, u\right)=0, \quad \text { where } \quad \mathcal{F}\left(v_{+}, u\right):=u-\mathbb{T}_{\theta_{0}^{1+\alpha}} \overline{\mathcal{B}}(u)-\mathcal{S}_{+} v_{+},
$$

where $v_{+} \in \Phi_{+}$and $u \in \mathbb{L}_{\theta_{0}}$. Then, on the one hand, according to (6.10) and Lemmas 6.1 and 6.2 the function $\mathcal{F}$ is well defined (recall that $\theta_{0}^{1+\alpha}>r_{0}\left(S_{0}\right)$ ) and belongs to the class $C^{1, \alpha}\left(\Phi_{+} \times \mathbb{L}_{\theta_{0}}, \mathbb{L}_{\theta_{0}}\right)$. Moreover, $\mathcal{F}_{u}^{\prime}(0,0)=\mathrm{Id}$. On the other hand, it is obvious that every solution of (6.20) satisfies (6.3).

Thus, applying the implicit function theorem to (6.20) we construct a $C^{1, \alpha}$-mapping $\mathbb{V}: B\left(\rho, 0, \Phi_{+}\right) \rightarrow \mathbb{L}_{\theta_{0}}$, where $\rho$ is a sufficiently small positive number such that

$$
\mathcal{F}\left(v_{+}, \mathbb{V}\left(v_{+}\right)\right) \equiv 0 \quad \forall v_{+} \in B\left(\rho, 0, \Phi_{+}\right)
$$


Moreover, since $\mathbb{V}(0)=0$, we have

$$
\left\|\mathbb{V}\left(v_{+}\right)\right\|_{\mathbb{L}_{\theta_{0}}} \leq C\left\|v_{+}\right\|_{\Phi_{+}} \quad \forall v_{+} \in B\left(\rho, 0, \Phi_{+}\right) .
$$

It remains to verify (6.8). Now, it follows from (6.21), (6.22), and Lemmas 6.1 and 6.2 that

$$
\begin{aligned}
\left\|\mathbb{V}_{n}\left(v_{+}\right)-S_{+}^{n} v_{+}\right\|_{\Phi} & \leq \theta_{0}^{(1+\alpha) n}\left\|\mathbb{V}\left(v_{+}\right)-\mathcal{S}_{+} v_{+}\right\|_{\mathbb{L}_{\theta_{0}^{1+\alpha}}} \\
& =\theta_{0}^{(1+\alpha) n}\left\|\mathbb{T}_{\theta_{0}^{1+\alpha}} \overline{\mathcal{B}}\left(\mathbb{V}\left(v_{+}\right)\right)\right\|_{\mathbb{L}_{\theta_{0}^{1+\alpha}}} \\
& \leq C_{1} \theta_{0}^{(1+\alpha) n}\left\|\overline{\mathcal{B}}\left(\mathbb{V}\left(v_{+}\right)\right)\right\|_{\mathbb{L}_{\theta_{0}^{1+\alpha}}} \\
& \leq C_{2} \theta_{0}^{(1+\alpha) n}\left\|\mathbb{V}\left(v_{+}\right)\right\|_{\mathbb{L}_{\theta_{0}}}^{1+\alpha} \\
& \leq C_{3} \theta_{0}^{(1+\alpha) n}\left\|v_{+}\right\|_{\Phi}^{1+\alpha}
\end{aligned}
$$

Theorem 6.1 is proved.

Corollary 6.1. Suppose that the hypotheses of Theorem 6.1 hold. Define the set

$$
\mathcal{V}_{0}:=\left\{\mathbb{V}_{0}\left(v_{+}\right), v_{+} \in B\left(\rho, 0, \Phi_{+}\right)\right\} .
$$

Then $\mathcal{V}_{0}$ is a smooth $\left(C^{1, \alpha}\right.$-smooth $)$ submanifold in $\Phi$ that is diffeomorphic to $\Phi_{+}$. Moreover,

$$
\left\|\mathbb{V}_{0}\left(v_{+}\right)-v_{+}\right\|_{\Phi} \leq C\left\|v_{+}\right\|_{\Phi}^{1+\alpha} \quad \forall v_{+} \in B\left(\rho, 0, \Phi_{+}\right)
$$

and therefore the tangent plane to the manifold $\mathcal{V}_{0}$ at the origin coincides with $\Phi_{+}$.

Indeed, from (6.21) we see that $\mathbb{V}_{0}^{\prime}(0)=\operatorname{Id}_{\Phi_{+}}$. Therefore, by the implicit function

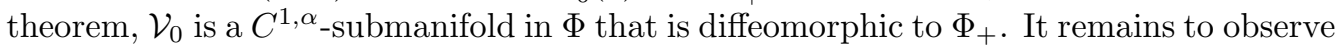
that (6.24) is an immediate consequence of inequality (6.8).

Remark 6.1. Note that by construction the solution $u_{n}:=\mathbb{V}_{n}\left(v_{+}\right)$of equation (6.3) belongs to $\mathbb{L}_{\theta_{0}}$ and is therefore decreasing as $n \rightarrow-\infty$ at least as fast as $\theta_{0}^{n}$ (for $\theta_{0}>1$ ). Therefore we call $\mathcal{V}_{0}$ a strongly unstable manifold. On the other hand, the sequence $v_{n}:=\mathcal{S}_{+}^{n} v_{+}$can be interpreted as the unique solution of the linear difference equation

$$
v_{n}=S_{0} v_{n-1}, \quad v_{0}=v_{+}, \quad v_{n} \in \Phi_{+}, \quad n \in \mathbb{Z}_{-} .
$$

Moreover, it follows from (6.10) and (6.25) that

$$
C_{\varepsilon}\left(r_{0}+\varepsilon\right)^{n}\left\|v_{+}\right\|_{\Phi} \leq\left\|v_{n}\right\|_{\Phi} \leq C_{1} \theta_{0}^{n}\left\|v_{+}\right\|_{\Phi} \quad \forall \varepsilon>0,
$$

where $C_{1}$ and $C_{\varepsilon}$ are independent of $v_{+}$. Since $\theta_{0}^{1+\alpha}>r_{0}$, it follows from (6.8) and (6.26) that the solution $u_{n}:=\mathbb{V}_{n}\left(v_{+}\right)$of equation (6.3) is attracted to the solution $v_{n}=\mathcal{S}_{+}^{n} v_{+}$ of the linear equation (6.25). The following result shows that the solution $u_{n}=\mathbb{V}_{n}\left(v_{+}\right)$ is uniquely characterized by this property.

Corollary 6.2. Suppose that the hypotheses of Theorem 6.1 hold. Then there exists a positive number $\delta_{0}$ such that if $u=\left\{u_{n}\right\}_{n=0}^{-\infty}$ is a solution of equation (6.3) such that

$$
\|u\|_{\mathbb{L}_{\theta_{0}}} \leq \delta_{0}
$$

and there exists

$$
v_{+} \in B\left(\rho, 0, \Phi_{+}\right)
$$

satisfying

$$
u-v \in \mathbb{L}_{\theta_{0}^{1+\alpha}}, \quad v:=\mathcal{S}_{+} v_{+},
$$

then $u_{n}=\mathbb{V}_{n}\left(v_{+}\right)$and, in particular, $u_{0} \in \mathcal{V}_{0}$. 
Since $u-v \in \mathbb{L}_{\theta_{0}^{1+\alpha}}$ and $u-v$ satisfies the equation

$$
u_{n}-v_{n}-S_{0}\left(u_{n}-v_{n}\right)=\mathcal{B}\left(u_{n}\right),
$$

by Lemmas 6.1 and 6.2 we can take the inverse of the linear part of equation (6.27). Thus, the pair $\left(u, v_{+}\right)$satisfies (6.20). Since $\mathbb{V}\left(v_{+}\right)$, the solution of (6.20) constructed by the implicit function theorem, is unique in a small neighborhood, we have $u=\mathbb{V}\left(v_{+}\right)$if the constant $\delta_{0}$ is sufficiently small.

Corollary 6.3. The manifold $\mathcal{V}_{0}$ is locally invariant under the mapping $S_{0}$, that is, there exists a positive number $\delta_{0}^{\prime}$ such that if $w \in \mathcal{V}_{0}$ and $\|w\|_{\Phi} \leq \delta_{0}^{\prime}$, then $S_{0} w \in \mathcal{V}_{0}$.

This assertion is an obvious consequence of the uniqueness of the solution $\mathbb{V}\left(v_{+}\right)$proved in Corollary 6.2.

Remark 6.2. Note that, by contrast with the standard theorems on unstable manifolds (see, for example, [1 39]), we have assumed neither that the zero equilibrium is hyperbolic nor that the spectral projection onto the subspace $\Phi_{+}$exists. We have not even assumed that there is a complement to the space $\Phi_{+}$in $\Phi$.

To conclude this section we state two more important corollaries of the theorem proved above.

Corollary 6.4. Suppose that the hypotheses of Theorem 6.1 hold and let $T: \Phi \rightarrow \Phi$ be some (linear) isometry of the Banach space $\Phi$ that commutes with the operator $S$, that is, $T \circ S=S \circ T$. Suppose that the subspace $\Phi_{+}$is invariant under this isometry: $T \Phi_{+} \subset \Phi_{+}$. Then the mapping $\mathbb{V}$ constructed in Theorem 6.1 is also invariant under the isometry $T$ :

$$
\mathbb{V}_{n}\left(T v_{+}\right)=T \mathbb{V}_{n}\left(v_{+}\right) \quad \forall v_{+} \in B\left(\rho, 0, \Phi_{+}\right), \quad n \in \mathbb{Z}_{-} .
$$

Indeed, along with $\mathbb{V}_{n}\left(v_{+}\right), n \in \mathbb{Z}_{-}$, the sequence $T^{-1} \mathbb{V}_{n}\left(T v_{+}\right)$is also a solution of equation (6.3), and it also tends to $v_{n}:=\mathcal{S}_{+}^{n} v_{+}$as $n \rightarrow-\infty$. Hence by Corollary 6.2 we have $T^{-1} \mathbb{V}_{n}\left(T v_{+}\right)=\mathbb{V}_{n}\left(v_{+}\right)$.

Corollary 6.5. Suppose that the hypotheses of Theorem 6.1 hold and let $\Phi_{1}$ be some other Banach space containing the space $\Phi: \Phi \subset \Phi_{1}$. Suppose also that the linear operator $S_{0}$ can be extended to a continuous linear operator $\widetilde{S}_{0}$ in $\Phi_{1}$ such that

$$
\theta_{0}^{1+\alpha}>r_{0}\left(\widetilde{S}_{0}\right)
$$

and the sequence $S_{+}^{n} v_{+}$admits the estimate

$$
\left\|S_{+}^{n} v_{+}\right\|_{\Phi_{1}} \leq C \theta_{0}^{n}\left\|v_{+}\right\|_{\Phi_{1}} \quad \forall v_{+} \in B\left(2 \rho, 0, \Phi_{+}\right) .
$$

Finally, suppose that the nonlinear operator $\mathcal{B}(u)$ admits the estimate

$$
\begin{aligned}
\left\|\mathcal{B}(u)-\mathcal{B}\left(u_{1}\right)\right\|_{\Phi_{1}} & \leq C_{1}\left(\|u\|_{\Phi}^{\alpha}+\left\|u_{1}\right\|_{\Phi}^{\alpha}\right)\left\|u-u_{1}\right\|_{\Phi_{1}} \\
\forall u, u_{1} & \in B\left(R_{0}, 0, \Phi\right),
\end{aligned}
$$

where $B\left(R_{0}, 0, \Phi\right)$ is an $R_{0}$-ball in the space $\Phi$ containing the manifold $\mathcal{V}_{0}$. Then there exists a positive number $\rho_{0} \leq \rho$, depending on the constants $C$ and $C_{1}$, such that

$$
C_{2}^{-1}\left\|v_{+}-w_{+}\right\|_{\Phi_{1}} \leq\left\|\mathbb{V}_{0}\left(v_{+}\right)-\mathbb{V}_{0}\left(w_{+}\right)\right\|_{\Phi_{1}} \leq C_{2}\left\|v_{+}-w_{+}\right\|_{\Phi_{1}}
$$

for any $v_{+}, w_{+} \in B\left(\rho_{0}, 0, \Phi_{+}\right)$and some constant $C_{2}$ depending on $C$ and $C_{1}$.

Proof. By construction the sequences $\mathbb{V}\left(v_{+}\right)$and $\mathbb{V}\left(w_{+}\right)$satisfy the relation

$$
\mathbb{V}\left(v_{+}\right)-\mathbb{V}\left(w_{+}\right)+\mathcal{S}_{+}\left(v_{+}-w_{+}\right)=\mathbb{T}_{\theta_{0}^{1+\alpha}}\left[\overline{\mathcal{B}}\left(\mathbb{V}\left(v_{+}\right)\right)-\overline{\mathcal{B}}\left(\mathbb{V}\left(w_{+}\right)\right)\right]
$$


It follows from (6.29) that the operator $\mathbb{T}_{\theta_{0}^{1+\alpha}}$ can be extended to a continuous linear operator in $\mathbb{L}_{\theta^{1+\alpha}}\left(\Phi_{1}\right)$. Hence, (6.33), (6.31), and (6.22) imply the estimate

$$
\begin{aligned}
\| \mathbb{T}_{\theta_{0}^{1+\alpha}}[\overline{\mathcal{B}} & \left.\left.\mathbb{V}\left(v_{+}\right)\right)-\overline{\mathcal{B}}\left(\mathbb{V}\left(w_{+}\right)\right)\right] \|_{\mathbb{L}_{\theta_{0}^{1+\alpha}}\left(\Phi_{1}\right)} \\
& \leq C^{\prime}\left\|\overline{\mathcal{B}}\left(\mathbb{V}\left(v_{+}\right)\right)-\overline{\mathcal{B}}\left(\mathbb{V}\left(w_{+}\right)\right)\right\|_{\mathbb{L}_{\theta_{0}^{1+\alpha}}\left(\Phi_{1}\right)} \\
& \leq C^{\prime \prime}\left(\left\|\mathbb{V}\left(v_{+}\right)\right\|_{\mathbb{L}_{\theta_{0}}}^{\alpha}+\left\|\mathbb{V}\left(w_{+}\right)\right\|_{\mathbb{L}_{\theta_{0}}^{\alpha}}^{\alpha}\left\|\mathbb{V}\left(v_{+}\right)-\mathbb{V}\left(w_{+}\right)\right\|_{\mathbb{L}_{\theta_{0}}\left(\Phi_{1}\right)}\right. \\
& \leq C^{\prime \prime \prime} \rho_{0}^{\alpha}\left\|\mathbb{V}\left(v_{+}\right)-\mathbb{V}\left(w_{+}\right)\right\|_{\mathbb{L}_{\theta_{0}}\left(\Phi_{1}\right)},
\end{aligned}
$$

for any $v_{+}, w_{+} \in B\left(\rho_{0}, 0, \Phi_{+}\right)$, where the constants $C^{\prime}, C^{\prime \prime}$, and $C^{\prime \prime \prime}$ depend only on $C_{1}$ and the norm of the operator $\mathbb{T}_{\theta_{0}^{1+\alpha}}$ in the space $\mathbb{L}_{\theta^{1+\alpha}}\left(\Phi_{1}\right)$ (but are independent of $C$ ). Assuming that $\rho_{0}>0$ satisfies the inequality $C^{\prime \prime \prime} \rho_{0}^{\alpha} \leq \frac{1}{2}$ and using the obvious embedding $\mathbb{L}_{\theta_{0}^{1+\alpha}}\left(\Phi_{1}\right) \subset \mathbb{L}_{\theta_{0}}\left(\Phi_{1}\right)$ and (6.33) we now obtain the inequality

$$
\frac{1}{2}\left\|\mathcal{S}_{+}\left(v_{+}-w_{+}\right)\right\|_{\mathbb{L}_{\theta_{0}}\left(\Phi_{1}\right)} \leq\left\|\mathbb{V}\left(v_{+}\right)-\mathbb{V}\left(w_{+}\right)\right\|_{\mathbb{L}_{\theta_{0}}\left(\Phi_{1}\right)} \leq \frac{3}{2}\left\|\mathcal{S}_{+}\left(v_{+}-w_{+}\right)\right\|_{\mathbb{L}_{\theta_{0}}\left(\Phi_{1}\right)}
$$

valid for any $v_{+}, w_{+} \in B\left(\rho_{0}, 0, \Phi_{+}\right)$. The right-hand inequality in (6.32) is an immediate consequence of (6.34) and (6.30). To prove the left-hand inequality we observe that by $(6.33),(6.34)$, and $(6.30)$ we have

$$
\begin{aligned}
\left\|\mathbb{V}_{0}\left(v_{+}\right)-\mathbb{V}_{0}\left(w_{+}\right)-\left(v_{+}-w_{+}\right)\right\|_{\Phi_{1}} & \leq\left\|\mathbb{V}\left(v_{+}\right)-\mathbb{V}\left(w_{+}\right)-\mathcal{S}_{+}\left(v_{+}-w_{+}\right)\right\|_{\mathbb{L}_{\theta_{0}}\left(\Phi_{1}\right)} \\
& \leq C^{\prime \prime \prime} \rho_{0}^{\alpha}\left\|\mathbb{V}\left(v_{+}\right)-\mathbb{V}\left(w_{+}\right)\right\|_{\mathbb{L}_{\theta_{0}}\left(\Phi_{1}\right)} \\
& \leq \frac{3}{2} C^{\prime \prime \prime} \rho_{0}^{\alpha}\left\|\mathcal{S}_{+}\left(v_{+}-w_{+}\right)\right\|_{\mathbb{L}_{\theta_{0}}\left(\Phi_{1}\right)} \\
& \leq \frac{3}{2} C C^{\prime \prime \prime} \rho_{0}^{\alpha}\left\|v_{+}-w_{+}\right\|_{\Phi_{1}}
\end{aligned}
$$

Finally, fixing $\rho_{0}>0$ so that $\frac{3}{2} C C^{\prime \prime \prime} \rho_{0}^{\alpha}=\frac{1}{2}$ we obtain

$$
\left\|\mathbb{V}_{0}\left(v_{+}\right)-\mathbb{V}_{0}\left(w_{+}\right)\right\|_{\Phi_{1}} \geq \frac{1}{2}\left\|v_{+}-w_{+}\right\|_{\Phi_{1}} .
$$

Corollary 6.5 is proved.

Remark 6.3. In what follows we will take $\Phi$ to be $L^{\infty}\left(\mathbb{R}^{n}\right) ; \Phi_{1}$ to be the weighted space $L_{\phi}^{\infty}\left(\mathbb{R}^{n}\right) ; S$ to be the solution operator of equation (0.1) per unit time; and the isometry $T$ to be the group $T_{h}$ of spatial translations.

\section{Strongly unstable manifolds of ReaCtion-Diffusion equations AND LOWER BOUNDS FOR THE $\varepsilon$-ENTROPY OF THE ATTRACTOR}

In this section we apply the abstract construction of a strongly unstable manifold to the study of equation (0.1). To carry out this program we shall need the following classical functional spaces.

Definition 7.1. For any $\sigma>0$ let $\mathbb{B}_{\sigma}=\mathbb{B}_{\sigma}\left(\mathbb{R}^{n}, \mathbb{C}\right)$ denote the subspace of $L^{\infty}\left(\mathbb{R}^{n}, \mathbb{C}\right)$ consisting of functions $u$ whose Fourier transform $\widehat{u}$ has compact support contained in the cube $[-\sigma, \sigma]^{n}$ :

$$
\mathbb{B}_{\sigma}:=\left\{u \in L^{\infty}\left(\mathbb{R}^{n}, \mathbb{C}\right), \operatorname{supp} \widehat{u} \subset[-\sigma, \sigma]^{n}\right\} .
$$

We also introduce the subspace $\mathbb{B}_{\sigma}^{\mathrm{Re}}\left(\mathbb{R}^{n}, \mathbb{R}\right) \subset \mathbb{B}_{\sigma}$ whose elements are real-valued functions: $\mathbb{B}_{\sigma}^{\operatorname{Re}}:=\operatorname{Re} \mathbb{B}_{\sigma}$.

It is well known that $\mathbb{B}_{\sigma, \xi_{0}}$ is the space of holomorphic functions with exponential growth (see, for example, [7, 15]). 
We also need the following subclass of weight functions of exponential growth.

Definition 7.2. A function $\phi \in C_{\mathrm{loc}}\left(\mathbb{R}^{n}\right)$ is a weight function with polynomial growth of degree $N \in \mathbb{R}_{+}$if the following conditions hold:

$$
\phi(x)>0, \quad \phi(x+y) \leq C_{\phi}(1+|y|)^{N} \phi(x) \quad \forall x, y \in \mathbb{R}^{n} .
$$

It is obvious that any weight function with polynomial growth is a weight function with exponential growth rate $\varepsilon$ (for any $\varepsilon>0$ ). The following functions are polynomial analogues of the functions (1.10):

$$
\psi_{l, x_{0}}(x):=\left(1+\left|x-x_{0}\right|\right)^{-l}, \quad x_{0} \in \mathbb{R}, \quad l \in \mathbb{R}, \quad l \neq 0 .
$$

It is easy to verify that these functions satisfy (7.2) with exponent $N=|l|$ and constant $C_{\psi_{l, x_{0}}}=1$. Moreover, all the properties of weight functions of exponential growth stated in Proposition 1.2 have obvious analogues in the case of functions of polynomial growth. For example, the following proposition is an analogue of item 5 of Proposition 1.2.

Proposition 7.1. Let $\phi$ be a weight function with polynomial growth of degree $N$. Then for any $u \in L_{\phi}^{\infty}\left(\mathbb{R}^{n}\right)$ we have the inequality

$$
\|u\|_{L_{\phi}^{\infty}\left(\mathbb{R}^{n}\right)} \leq \sup _{y \in \mathbb{R}^{n}}\left\{\phi(y)\|u\|_{L_{\psi_{N, y}}^{\infty}\left(\mathbb{R}^{n}\right)}\right\} \leq C_{\phi}\|u\|_{L_{\phi}^{\infty}\left(\mathbb{R}^{n}\right)},
$$

where the function $\psi_{N, y}(x)$ is defined in formula (7.3).

The proof of (7.4) repeats word for word the proof of (1.28).

We are now ready to state the main result of this section.

Theorem 7.1. Suppose that the hypotheses of Theorem 1.1 hold. Suppose also that

$$
f(0)=0 \quad \text { and } \quad \sigma\left(-f^{\prime}(0)-\lambda_{0}, \mathbb{R}^{k}\right) \cap\{\operatorname{Im} \lambda>0\} \neq \varnothing .
$$

Then there exists a complex vector $e \in \mathbb{C}^{k}$ and positive numbers $\sigma$ and $\rho_{0}$ defining the subspace

$$
\Phi_{+}=\Phi_{+}(\sigma):=\operatorname{Re}\left[\mathbb{B}_{\sigma} \cdot e\right]
$$

and a smooth $\left(C^{1,1}\right.$-smooth $)$ mapping

$$
\mathbb{V}_{0}: B\left(\rho_{0}, 0, \Phi_{+}\right) \rightarrow \mathcal{A} \quad \text { such that } \quad T_{h} \circ \mathbb{V}_{0}=\mathbb{V}_{0} \circ T_{h} \quad \forall h \in \mathbb{R}^{n}
$$

and

$$
\left\|\mathbb{V}_{0}(v)-v\right\|_{L^{\infty}\left(\mathbb{R}^{n}\right)} \leq C\|v\|_{L^{\infty}\left(\mathbb{R}^{n}\right)}^{2} \quad \forall v \in B\left(\rho_{0}, 0, \Phi_{+}\right) .
$$

Moreover, for any weight function $\phi$ with polynomial growth

$$
C_{1}^{-1}\left\|v_{1}-v_{2}\right\|_{L_{\phi}^{\infty}\left(\mathbb{R}^{n}\right)} \leq\left\|\mathbb{V}_{0}\left(v_{1}\right)-\mathbb{V}_{0}\left(v_{2}\right)\right\|_{L_{\phi}^{\infty}\left(\mathbb{R}^{n}\right)} \leq C_{1}\left\|v_{1}-v_{2}\right\|_{L_{\phi}^{\infty}\left(\mathbb{R}^{n}\right)}
$$

for any $v_{1}, v_{2} \in B\left(\rho_{0}, 0, \Phi_{+}\right)$, where the constant $C_{1}$ depends only on the constants $N$ and $C_{\phi}$ in inequality (7.2) and is independent of the explicit form of the weight function $\phi$.

Proof. We wish to construct a strongly unstable manifold of the zero equilibrium for the solution operator $S=S_{1}: \Phi_{b} \rightarrow \Phi_{b}$ of equation (0.1) per unit time. To do this we need to verify that the hypotheses of Theorem 6.1 hold for this particular case. Now, in this case, $\Phi=\Phi_{b}:=L^{\infty}\left(\mathbb{R}^{n}\right), \quad S_{0}:=D_{u_{0}} S_{1}(0)$, and $\mathcal{B}(u):=S(u)-S_{0}(u)$, where $D_{u_{0}} S_{t}(0)$ is the solution operator of the linear parabolic equation

$$
\partial_{t} v=a \Delta_{x} v-\left(\vec{L}, \nabla_{x}\right) v-f^{\prime}(0) v-\lambda_{0} v,\left.\quad v\right|_{t=0}=v_{0},
$$

which is the linearization of the problem $(0.1)$ in a neighborhood of the zero equilibrium. Then, by Corollary $1.3, \mathcal{B} \in C^{1,1}\left(\Phi_{b}, \Phi_{b}\right)$ and $\mathcal{B}(0)=\mathcal{B}^{\prime}(0)=0$. Hence condition (6.1) is 
satisfied. Moreover, by (1.30) for any weight function with a sufficiently small exponential growth rate we have

$$
\left\|\mathcal{B}(u)-\mathcal{B}\left(u_{1}\right)\right\|_{\Phi_{\phi}} \leq C\left(\|u\|_{\Phi_{b}}+\left\|u_{1}\right\|_{\Phi_{b}}\right)\left\|u-u_{1}\right\|_{\Phi_{\phi}},
$$

where $C$ depends on the norms of $u$ and $u_{1}$ and the constant $C_{\phi}$ in inequality (1.24) (but is independent of the explicit form of $\phi$ ). Hence (6.31) is also satisfied for $\Phi_{1}=\Phi_{\phi}$. Thus, it remains to verify the conditions on the linear operator $S_{0}$. For that we need the following lemmas.

Lemma 7.1. The spectral radius of the operator $S_{0}$ is calculated by the formula

$$
r_{0}\left(S_{0}\right)=e^{\max \operatorname{Re} \sigma\left(-f^{\prime}(0)-\lambda_{0}, \mathbb{R}^{k}\right)}
$$

and therefore there exists an eigenvector $e \in \mathbb{C}^{k}$ of the operator $-f^{\prime}(0)-\lambda_{0}$ corresponding to an eigenvalue $\Lambda_{0}$ such that

$$
r_{0}\left(S_{0}\right)=e^{\operatorname{Re} \Lambda_{0}}, \quad\left(-f^{\prime}(0)-\lambda_{0}\right) e=\Lambda_{0} e .
$$

Proof. Since the function $v(t):=e^{\Lambda t} e_{\Lambda}$ is a spatially homogeneous solution of equation (7.10) for any $\Lambda \in \sigma\left(-f^{\prime}(0)-\lambda_{0}\right)$ and the corresponding eigenvector $e_{\Lambda} \in \mathbb{C}^{k}$, we have

$$
r_{0}\left(S_{0}\right) \geq e^{\max \operatorname{Re} \sigma\left(-f^{\prime}(0)-\lambda_{0}, \mathbb{R}^{k}\right)} .
$$

We now prove the reverse inequality. We set $M_{0}:=\max \operatorname{Re} \sigma\left(-f^{\prime}(0)-\lambda_{0}, \mathbb{R}^{k}\right)$. For any $\delta>0$ we introduce an equivalent norm in the space $\mathbb{R}^{k}$ by the following standard formula:

$$
\|v\|_{\delta}^{2}:=\int_{0}^{\infty} e^{\left(-M_{0}-\delta-A\right) t} v \cdot v d t, \quad A:=f^{\prime}(0)+\lambda_{0} .
$$

The integral on the right-hand side of (7.14) converges due to the fact that the spectrum of the operator $-M_{0}-\delta-A$ is contained in the negative half-plane. Moreover, it is easy to verify that

$$
(A v, v)_{\delta} \geq-\left(M_{0}+\delta\right)\|v\|_{\delta}^{2} \quad \forall v \in \mathbb{R}^{k} .
$$

For, since the norm (7.14) does not increase along trajectories of the semigroup $s_{t}:=$ $e^{\left(-M_{0}-\delta-A\right) t}$, we have

$$
\left.\frac{d}{d t}\left(\left\|s_{t} v\right\|_{\delta}^{2}\right)\right|_{t=0}=2\left(\left(-M_{0}-\delta-A\right) v, v\right)_{\delta} \leq 0,
$$

and inequality (7.15) follows.

We now introduce the function $w(t, x):=\|v(t, x)\|_{\delta}^{2}$, where $v(t)$ is an arbitrary solution of (7.10). Then, similarly to (1.2), this function satisfies the inequality

$$
\begin{gathered}
\partial_{t} w-a \Delta_{x} w+\left(\vec{L}, \nabla_{x}\right) w-2\left(M_{0}+\delta\right) w \leq 0, \\
\left.w\right|_{t=0}=\left\|v_{0}\right\|_{\delta}^{2},\left.\quad w\right|_{\partial \Omega}=0 .
\end{gathered}
$$

Applying the classical maximum principle to equation (7.16) we obtain

$$
\|w(t)\|_{L^{\infty}} \leq e^{2\left(M_{0}+\delta\right) t}\|w(0)\|_{L^{\infty}}
$$

and, consequently, $r_{0}\left(S_{0}\right) \leq M_{0}+\delta$. Since $\delta>0$ is arbitrary, (7.12) is proved. The existence of an eigenvector $e$ and an eigenvalue $\Lambda_{0}$ satisfying (7.13) follows directly from (7.12). Lemma 7.1 is proved.

Thus, if (7.5) holds, then (6.2) holds. The following lemma gives an estimate for the spectrum of the operator $S_{0}$ in the weighted spaces $\Phi_{\phi}$. 
Lemma 7.2. Let $\phi$ be a weight function with exponential growth rate $\alpha>0$ and let $\widetilde{S}_{0}$ be an extension of the operator $S_{0}$ to a continuous operator in $\Phi_{\phi}$. Then

$$
r_{0}\left(\widetilde{S}_{0}, \Phi_{\phi}\right) \leq r_{0}\left(S_{0}, \Phi_{b}\right)+C \alpha,
$$

where the constant $C$ is independent of $\alpha, C_{\phi}$, and the explicit form of the weight function $\phi$.

Proof. Observe that the operator of multiplication by the weight function $\phi^{-1}$ realizes an isomorphism between the spaces $L^{\infty}\left(\mathbb{R}^{n}\right)$ and $L_{\phi}^{\infty}\left(\mathbb{R}^{n}\right)$. Moreover, in the case of the weight functions (1.10) the operator $S_{\alpha, x_{0}}:=\phi_{\alpha, x_{0}} \circ \widetilde{S}_{0} \circ \phi_{\alpha, x_{0}}^{-1}$ is the solution operator of a linear parabolic equation of the form (1.11). Hence, similarly to the proof of Lemma 7.1, the estimates (1.14) and the maximum principle imply that

$$
r_{0}\left(\widetilde{S}_{0}, \Phi_{\phi_{\alpha, x_{0}}}\right)=r_{0}\left(\widetilde{S}_{\alpha, x_{0}}, \Phi_{b}\right) \leq r_{0}\left(S_{0}, \Phi_{b}\right)+C \alpha .
$$

One can derive formula (7.17) for an arbitrary weight $\phi$ from this special case in a standard way using the estimates (1.28) and the invariance of $S_{0}$ under the group of spatial translations. Lemma 7.2 is proved.

Thus, condition (6.29) follows from condition (6.6) (for the case of the space $\Phi_{1}=\Phi_{\phi}$ with a weight function of a sufficiently small exponential growth rate). It remains to verify the conditions on the invariant spectral subspace $\Phi_{+}$.

Lemma 7.3. There exist $\theta_{0}>1$ satisfying condition (6.6), $\sigma>0$, and an invariant subspace $\Phi_{+}$(under $S_{0}$ and $T_{h}$ ) defined by formula (7.6), where the vector e satisfies (7.13), such that the restriction $S_{+}$of the operator $S_{0}$ to the invariant subspace $\Phi_{+}$is invertible and satisfies (6.5). Moreover, for any $N>0$ there exists $\varepsilon_{N}$ such that

$$
\left\|\mathcal{S}_{+}^{-l} v_{+}\right\|_{\Phi_{\psi_{\varepsilon}, N, x_{0}}} \leq C \theta_{0}^{-l}\left\|v_{+}\right\|_{\Phi_{\psi_{\varepsilon}, N, x_{0}}} \quad \forall v_{+} \in \Phi_{+}, \quad l \in \mathbb{N},
$$

where $\psi_{\varepsilon, N, x_{0}}(x):=\left(1+\varepsilon\left|x-x_{0}\right|^{-N}\right), \quad 0<\varepsilon \leq \varepsilon_{N}$, and the constant $C$ is independent of $N, \varepsilon \leq \varepsilon_{N}$, and $x_{0}$.

Proof. Recall that $S_{0}$ is the solution operator per unit time for equation (7.10). Therefore, applying the Fourier transformation to (7.10) we obtain

$$
\widehat{S_{0}^{l} v}(\xi)=e^{\left(-a|\xi|^{2}-i \vec{L} \cdot \xi-\lambda_{0}-f^{\prime}(0)\right) l} \widehat{v}_{0}(\xi), \quad \xi \in \mathbb{R}^{n}, \quad l \in \mathbb{N} .
$$

Moreover, (7.20) and the definitions of the eigenvalue $\Lambda_{0}$ and the subspace $\Phi_{+}$imply the simpler formula

$$
\widehat{S_{0}^{l} v_{+}}(\xi)=e^{\left(-a|\xi|^{2}-i \vec{L} \cdot \xi+\Lambda_{0}\right) l} \widehat{v}_{+}, \quad l \in \mathbb{N},
$$

for the case of the initial conditions $v_{+} \in \Phi_{+}$. Formula (7.21) implies, in particular, the invariance of the space $\Phi_{+}$under the operator $S_{0}$. Moreover, since the Fourier transform of any function in $\Phi_{+}$has compact support, it follows from (7.21) that the operator $S_{0}$ is invertible on $\Phi_{+}$(for any choice of the exponent $\sigma$ ) and (7.21) is valid for any $l \in \mathbb{Z}$. Thus, it remains to find the exponent $\sigma>0$ so that (7.21) holds. We fix an (arbitrary) number $\theta_{0}>1$ satisfying (6.6), that is, $\theta_{0}^{2}>r_{0}\left(S_{0}\right)>\theta_{0}$; such $\theta_{0}$ exists by (7.5) and (7.12). We also fix a positive number $m_{0}$ so that the following inequality holds:

$$
\left|e^{-a|\xi|^{2}-i \vec{L} . \xi+\Lambda_{0}}\right|=e^{-a|\xi|^{2}} e^{\operatorname{Re} \Lambda_{0}}=e^{-a|\xi|^{2}} r_{0}\left(S_{0}\right)>\theta_{0} \quad \text { if } \quad|\xi| \leq m_{0},
$$

that is, $m_{0}<\sqrt{a^{-1} \ln \frac{r_{0}\left(S_{0}\right)}{\theta_{0}}}$. We claim that if the exponent $\sigma>0$ satisfies the condition

$$
[-\sigma, \sigma]^{n} \subset B_{0}^{m_{0} / 2}, \quad \text { that is, } \sqrt{n} \sigma \leq \frac{m_{0}}{2},
$$


then for any $v_{+} \in \Phi_{+}(\sigma)$ (7.19) holds. Now, by (7.21), (7.20) and (7.23), the solution $S_{+}^{l} v_{+}, v_{+} \in \Phi_{+}$, can be found as follows:

$$
\begin{aligned}
S_{+}^{-l} v_{+} & =\theta_{0}^{-l} \Phi_{l} * v_{+}, \quad l \in \mathbb{N}, \quad \text { where } \\
\widehat{\Phi}_{l}(\xi): & =e^{l\left[\ln \theta_{0}-\left(-a|\xi|^{2}-i \vec{L} . \xi+\Lambda_{0}\right)\right]} \psi(\xi) .
\end{aligned}
$$

Here the symbol $u * v$ denotes the convolution of $u$ and $v$ in $\mathbb{R}^{n}$, and the cut-off function $\psi \in C_{0}^{\infty}\left(\mathbb{R}^{n}\right)$ is such that $\psi(\xi)=1$ for $|\xi| \leq \frac{m_{0}}{2}$ and $\psi(\xi)=0$ for $|\xi| \geq m_{0}$. We observe that (7.22) implies that

$$
\ln \theta_{0}-\left(-a|\xi|^{2}-i \vec{L} . \xi+\Lambda_{0}\right) \leq-\mu_{0} \quad \forall \xi \in B_{0}^{m_{0}}
$$

for some positive $\mu_{0}$. Hence for any $N \in \mathbb{N}$

$$
\left\|\widehat{\Phi}_{l}\right\|_{C^{N}\left(\mathbb{R}^{n}\right)} \leq C_{N}
$$

and therefore

$$
\left|\Phi_{l}(x)\right| \leq C_{N}^{\prime}(1+|x|)^{-N},
$$

where the constants $C_{N}$ and $C_{N}^{\prime}$ depend on $N$, but do not depend on $l \in \mathbb{N}$. Now by $(7.24)$ and $(7.26)$ we have

$$
\begin{aligned}
\theta_{0}^{l}\left|\left(\mathcal{S}_{+}^{-l} v_{+}\right)(x)\right| & \leq\left(\int_{y \in \mathbb{R}^{n}}\left|\Phi_{l}(y)\right|^{1 / 2} d y\right) \sup _{y \in \mathbb{R}^{n}}\left\{\left|v_{+}(y)\right| \cdot\left|\Phi_{l}(x-y)\right|^{1 / 2}\right\} \\
& \leq C \sup _{y \in \mathbb{R}^{n}}\left\{\left|v_{+}(y)\right| \cdot\left|\Phi_{l}(x-y)\right|^{1 / 2}\right\},
\end{aligned}
$$

where the constant $C$ is independent of $l$ and $x$. Multiplying (7.27) by the function $\psi_{\varepsilon, N, x_{0}}(x)$, taking the supremum over $x \in \mathbb{R}^{n}$, and using (7.2) for the functions $\psi_{\varepsilon, N, x_{0}}(x)$ (obviously, we take the constant $C_{\psi_{\varepsilon, N, x_{0}}(x)}=1$ ) we obtain

$$
\begin{aligned}
\theta_{0}^{l} \mid\left(\mathcal{S}_{+} v_{+}\right) & \left.\right|_{\Phi_{\psi_{\varepsilon, N, x_{0}}}} \\
& \leq C \sup _{(x, y) \in \mathbb{R}^{2 n}}\left\{\left(1+\varepsilon\left|y-x_{0}\right|\right)^{-N}(1+\varepsilon|y-x|)^{N}\left|v_{+}(y)\right| \cdot\left|\Phi_{l}(x-y)\right|^{1 / 2}\right\} \\
& \leq C\left(\sup _{z \in \mathbb{R}^{n}}\left\{(1+\varepsilon|z|)^{N}\left|\Phi_{l}(z)\right|^{1 / 2}\right\}\right) \cdot\left\|v_{+}\right\|_{\Phi_{\varepsilon, N, x_{0}}} .
\end{aligned}
$$

Moreover, it is easy to deduce from (7.2) that for any $N \in \mathbb{R}_{+}$there exists a constant $\varepsilon_{N}=\varepsilon(N)>0$ and a constant $K$ independent of $N$ such that

$$
\sup _{z \in \mathbb{R}^{n}}\left\{(1+\varepsilon|z|)^{N}\left|\Phi_{l}(z)\right|^{1 / 2}\right\} \leq K
$$

for every $\varepsilon<\varepsilon_{N}$. Lemma 7.3 is proved.

Thus, we have verified all the hypotheses of Theorem 6.1. Hence there exists a strongly unstable manifold $\mathcal{V}_{0}$ of the operator $S$ corresponding to the invariant subspace $\Phi_{+}$ constructed in Lemma 7.3 and a $C^{1,1}$-mapping $\mathbb{V}_{0}: B\left(\rho, 0, \Phi_{+}\right) \rightarrow \mathcal{V}_{0}$ satisfying (7.8). Note also that to each $u_{0}:=\mathbb{V}_{0}\left(v_{+}\right), \quad v_{+} \in B\left(\rho, 0, \Phi_{+}\right)$there corresponds a complete trajectory $u(t) \in \mathcal{K}$ of equation (0.1) defined by

$$
u(t):= \begin{cases}S_{t-[t]} \mathbb{V}_{[t]}\left(v_{+}\right), & t \leq 0, \\ S_{t} u_{0}, & t \geq 0,\end{cases}
$$

where $[t]$ denotes the integer part of $t$, while $\mathbb{V}_{l}, l \in \mathbb{Z}_{-}$, are the operators constructed in Theorem 6.1. Hence, $\mathcal{V}_{0} \in \mathcal{A}$ by Theorem 2.1. Thus, it remains to verify estimate (7.9) using Corollary 6.5. To avoid the dependence of the radius $\rho_{0}$ on $N$, the exponent of the 
polynomial growth of the weight function $\phi$, we use the polynomial weights introduced in Lemma 7.3:

$$
\bar{\psi}_{N, x_{0}}(x):=\left(1+\varepsilon_{N}^{\prime}\left|x-x_{0}\right|\right)^{-N}, \quad N \in \mathbb{R}_{+}, \quad x_{0} \in \mathbb{R}^{n},
$$

where the small parameter $\varepsilon_{N}^{\prime}>0$ is chosen so that (7.19) holds, that is, $\varepsilon_{N}^{\prime} \leq \varepsilon_{N}$, and also (7.11) holds with the constant $C$ independent of $N$ and $x_{0}$. For that it suffices to choose $\varepsilon_{N}^{\prime}$ so that

$$
\bar{\psi}_{N, x_{0}}(x+y) \leq\left(1+\varepsilon_{N}^{\prime}|x|\right)^{N} \bar{\psi}_{N, x_{0}}(y) \leq e^{\alpha|x|} \bar{\psi}_{N, x_{0}}(y)
$$

for some sufficiently small $\alpha>0$. We now choose the weighted space $\Phi_{\bar{\psi}_{N, x_{0}}}$ to be the space $\Phi_{1}$ in Corollary 6.5. Then (6.31) follows immediately from (7.11). Moreover, by Lemma $7.2,(6.29)$ is also satisfied if the constant $\alpha>0$ is sufficiently small, and condition (6.30) follows from (7.19). Hence by Corollary 6.5 there exists $\rho_{0}>0$ independent of $N$ such that (7.9) holds for the weight functions $\phi=\bar{\psi}_{N, x_{0}}, N \in \mathbb{R}_{+}, x_{0} \in \mathbb{R}^{n}$, where the constant $C_{1}$ is independent of $x_{0}$. On the other hand, the functions (7.28) are obviously weight functions with polynomial growth of degree $N$ and

$$
C_{N}^{-1}\left(1+\left|x-x_{0}\right|\right)^{-N} \leq \bar{\psi}_{N, x_{0}}(x) \leq C_{N}\left(1+\left|x-x_{0}\right|\right)^{-N}, \quad \forall x \in \mathbb{R}^{n},
$$

where the constant $C_{N}$ depends on $N$, but is independent of $x_{0}$. Now it follows from (7.29) and Proposition 7.1 that (7.9) holds for any weight function with polynomial growth. Theorem 7.1 is proved.

The theorem we have just proved allows us to derive lower bounds for the $\varepsilon$-entropy of the attractor using the well-known estimates for the entropy in the spaces $\mathbb{B}_{\sigma}$.

Corollary 7.1. Suppose that the hypotheses of Theorem 7.1 hold. Then the $\varepsilon$-entropy of the restriction of the attractor $\mathcal{A}$ of equation (0.1) to the ball $B_{x_{0}}^{R}$ admits the estimate

$$
\mathbb{H}_{\varepsilon}\left(\left.\mathcal{A}\right|_{B_{x_{0}}^{R}}, L^{\infty}\left(B_{x_{0}}^{R}\right)\right) \geq C_{\mathcal{A}} R^{n} \ln _{+} \frac{R_{0}^{\prime}}{\varepsilon},
$$

where the constants $R_{0}^{\prime}>0$ and $C>0$ are independent of $\varepsilon, x_{0}$, and $R$. Moreover, the entropy of the restriction of the attractor to the unit ball satisfies the following sharper estimate: for any $\mu>0$ there exists $C_{\mu}>0$ such that

$$
\mathbb{H}_{\varepsilon}\left(\left.\mathcal{A}\right|_{B_{x_{0}}^{1}}, L^{\infty}\left(B_{x_{0}}^{1}\right)\right) \geq C_{\mu}\left(\ln \frac{R_{0}^{\prime}}{\varepsilon}\right)^{n+1-\mu} .
$$

Proof. We can assume without loss of generality that the eigenvector $e \in \mathbb{C}^{k}$ involved in (7.6), which gives the definition of the subspace $\Phi_{+}$, is normalized so that $|\operatorname{Re} e|=1$. (If $\operatorname{Re} e=0$, then one must take the vector $\bar{e}$ instead of the vector $e$.) Let $v_{1}, v_{2} \in$ $B\left(\delta, 0, \mathbb{B}_{\sigma}^{\mathrm{Re}}\right)$ for some $\delta \leq \rho_{0}$. Then using (7.8) we obtain the inequality

$$
\begin{aligned}
& \left\|\mathbb{V}_{0}\left(v_{1}\right)-\mathbb{V}_{0}\left(v_{2}\right)\right\|_{L^{\infty}\left(B_{x_{0}}^{R}\right)} \\
& \quad \geq\left\|v_{1}-v_{2}\right\|_{L^{\infty}\left(B_{x_{0}}^{R}\right)}-\left\|\mathbb{V}_{0}\left(v_{1}\right)-v_{1} \operatorname{Re} e\right\|_{\Phi_{b}}-\left\|\mathbb{V}_{0}\left(v_{2}\right)-v_{2} \operatorname{Re} e\right\|_{\Phi_{b}} \\
& \quad \geq\left\|v_{1}-v_{2}\right\|_{L^{\infty}\left(B_{x_{0}}^{R}\right)}-2 C \delta^{2}
\end{aligned}
$$

for any $R>0$ and $x_{0} \in \mathbb{R}^{n}$. Now let $\varepsilon>0$ be an arbitrary sufficiently small number and let $\delta=\frac{1}{2}(C \varepsilon)^{1 / 2}$. Then (7.32) and the embedding (7.7) yield the estimate

$$
\begin{aligned}
\mathbb{H}_{\frac{\varepsilon}{2}}\left(\mathbb{A}_{B_{x_{0}}^{R}}, L^{\infty}\left(B_{x_{0}}^{R}\right)\right) & \geq \mathbb{H}_{\varepsilon}\left(B\left(\frac{1}{2}(C \varepsilon)^{1 / 2}, 0, \mathbb{B}_{\sigma}^{\mathrm{Re}}\right), L^{\infty}\left(B_{x_{0}}^{R}\right)\right) \\
& =\mathbb{H}_{2 C^{-1 / 2} \varepsilon^{1 / 2}}\left(B\left(1,0, \mathbb{B}_{\sigma}^{\mathrm{Re}}\right), L^{\infty}\left(B_{x_{0}}^{R}\right)\right) .
\end{aligned}
$$


Therefore in order to derive estimates (7.30) and (7.31) it suffices to have analogous estimates for the $\varepsilon$-entropy of the unit ball in the spaces $\mathbb{B}_{\sigma}^{\mathrm{Re}}$. Proofs of these estimates can be found in [7] and [41], for example. Corollary 7.1 is proved.

Remark 7.1. The estimates (7.30) and (7.31) show that the upper bound (2.13) is sharp for all values of the parameters $\varepsilon, R$, and $x_{0}$. In particular, it follows from (7.31) that the restriction of the attractor $\mathcal{A}$ to any bounded domain has infinite fractal dimension. Note also that using a more accurate lower bound than (7.31) for the $\varepsilon$-entropy of the unit ball in the space $\mathbb{B}_{\sigma}^{\mathrm{Re}}$ one can obtain the following estimate for the entropy of the attractor:

$$
\mathbb{H}_{\varepsilon}\left(\left.\mathcal{A}\right|_{B_{x_{0}}^{1}}, L^{\infty}\left(B_{x_{0}}^{1}\right)\right) \geq C\left(\frac{\ln \frac{1}{\varepsilon}}{\left(\ln \ln \frac{1}{\varepsilon}\right)^{n}}\right)^{n+1},
$$

which differs from the upper bound (2.13) by a factor proportional to the double logarithm of $\varepsilon$. We will not use (7.34) in what follows and therefore do not give its proof.

Corollary 7.2. Suppose that the hypotheses of Theorem 7.1 hold. Then the generalized spatial topological entropy of the attractor $\mathcal{A}$ is strictly positive:

$$
0<C \leq \widehat{h}_{s p}\left(\mathcal{A}, T_{h}\right)<\infty
$$

and therefore

$$
h_{\text {top }}\left(\mathcal{A}, T_{h}\right)=\infty .
$$

We proved that $\widehat{h}_{s p}\left(\mathcal{A}, T_{h}\right)$ is finite in $\S 4$, and the fact that it is strictly positive follows from (7.30) and (5.20). The fact that the classical topological entropy of the action of the group $\left\{T_{h}, h \in \mathbb{R}^{n}\right\}$ is infinite is an immediate consequence of the fact that its generalized entropy is positive.

To conclude this section we state another useful corollary of the theorem proved above, which gives a model dynamical system for studying spatial chaos on the attractor.

Corollary 7.3. Suppose that the hypotheses of Theorem 7.1 hold. Then there exists a mapping

$$
\widetilde{\mathbb{V}}: \mathcal{B}(\sigma) \rightarrow \mathcal{A}, \quad \mathcal{B}(\sigma):=B\left(1,0, \mathbb{B}_{\sigma}^{\mathrm{Re}}\right),
$$

that commutes with the group $\left\{T_{h}, h \in \mathbb{R}^{n}\right\}$ of spatial translations and satisfies the estimate

$$
C_{1}^{-1}\left\|v_{1}-v_{2}\right\|_{L_{\phi}^{\infty}\left(\mathbb{R}^{n}\right)} \leq\left\|\widetilde{\mathbb{V}}\left(v_{1}\right)-\widetilde{\mathbb{V}}\left(v_{2}\right)\right\|_{L_{\phi}^{\infty}\left(\mathbb{R}^{n}\right)} \leq C_{1}\left\|v_{1}-v_{2}\right\|_{L_{\phi}^{\infty}\left(\mathbb{R}^{n}\right)}
$$

for any $v_{1}, v_{2} \in \mathcal{B}(\sigma)$, where the constant $C_{1}$ depends only on the constants $N$ and $C_{\phi}$ in (7.3) and is independent of the explicit form of the weight function $\phi$. In particular,

$$
\widehat{h}_{s p}\left(\widetilde{\mathbb{V}}(\mathcal{B}(\sigma)), T_{h}\right)=\widehat{h}_{s p}\left(\mathcal{B}(\sigma), T_{h}\right) .
$$

Indeed, it follows from the estimates (7.9) that we can take the mapping

$$
\widetilde{\mathbb{V}}(v):=\mathbb{V}_{0}\left(\rho_{0}^{-1} v \operatorname{Re} e\right) \quad \forall v \in \mathcal{B}(\sigma)
$$

for the required mapping $\widetilde{\mathbb{V}}$. (As in the proof of Corollary 7.1, we assume that $|\operatorname{Re} e|=1$.) Formula (7.38) is an immediate consequence of the "Lipschitzian" estimate (7.37) and the fact that the generalized spatial entropy is independent of the choice of the weight function. 
Remark 7.2. We have proved Theorem 7.1 under the assumption that $f(0)=0$ and the zero equilibrium of equation (0.1) is exponentially unstable. However, an analogous result holds under the weaker assumption that there exists at least one spatially homogeneous, exponentially unstable equilibrium $z_{0} \in \mathbb{R}^{k}$, that is, $f\left(z_{0}\right)+\lambda_{0} z_{0}=0$. Clearly, the change of variable $\widetilde{u}=u-z_{0}$ reduces this case to the hypotheses of Theorem 7.1.

Remark 7.3. In the special case under consideration where equation (0.1) has a scalar diffusion matrix $a \in \mathbb{R}_{+}$, Lemma 7.2 shows that (7.5) is equivalent to the exponential instability of the zero equilibrium. However, in the case of a more general diffusion matrix (for example, a diagonal matrix) (7.5) is not a necessary condition for the zero equilibrium to be exponentially unstable. Therefore, condition (7.5) of Theorem 7.1 is replaced by the condition

$$
\sigma\left(a \Delta_{x}-\left(\vec{L}, \nabla_{x}\right)-f^{\prime}(0)-\lambda_{0}, L^{2}\left(\mathbb{R}^{n}\right)\right) \cap\{\operatorname{Re} \lambda>0\} \neq \varnothing ;
$$

see $[24,43,44$.

\section{KOTEL'NIKOV'S FORMULA AND SPATIAL CHAOS IN REACTION-DIFFUSION EQUATIONS}

In the preceding section in Corollary 7.3 we constructed a Lipschitz embedding of the model group $\left(\mathcal{B}(\sigma), T_{h}\right)$ of spatial translations on the unit ball in the space $\mathbb{B}_{\sigma}$ into the group $\left(\mathcal{A}, T_{h}\right)$ of spatial translations acting on the attractor. In this section we study this model semigroup from the dynamic viewpoint. In particular, using a certain generalization of the classical Kotel'nikov-Cartwright formula for functions in $\mathbb{B}_{\sigma}$, we obtain a description of the dynamics generated by this semigroup in terms of embeddings of topological Bernoulli schemes. Note, however, that in contrast to the classical dynamical systems generated by systems of ordinary differential equations, the action of the group $T_{h}$ on $\mathbb{B}_{\sigma}$ (and, respectively, on the attractor $\mathcal{A}$ ) has infinite topological entropy (see Corollary 7.2). Therefore, classical Bernoulli schemes with finitely many symbols do not provide an adequate description of these dynamics, and we will use the following version of a Bernoulli scheme with infinitely many symbols.

Definition 8.1. Let $\mathcal{M}_{n}:=[-1,1]^{\mathbb{Z}^{n}}$. Then $\mathcal{M}_{n}$ endowed with the Tikhonov topology is a compact metrizable space, and the metric on it can be defined by the formula

$$
d_{\phi}\left(v_{1}, v_{2}\right)=\left\|v_{1}-v_{2}\right\|_{L_{\phi}^{\infty}\left(\mathbb{Z}^{n}\right)}:=\sup _{n \in \mathbb{Z}^{n}}\left\{\phi(n)\left|v_{1}(n)-v_{2}(n)\right|\right\}, \quad v_{1}, v_{2} \in \mathcal{M}_{n},
$$

where $\phi$ is an arbitrary weight function satisfying condition (2.2) and the space $\mathcal{M}_{n}$ is interpreted as the set of all mappings $v: \mathbb{Z}^{n} \rightarrow[-1,1]$.

We define the action of the group $\mathbb{Z}^{n}$ on $\mathcal{M}_{n}$ in the following standard way:

$$
\left(\mathcal{T}_{l} v\right)(m):=v(l+m), \quad v \in \mathcal{M}_{n}, \quad l, m \in \mathbb{Z}^{n} .
$$

We shall interprete the group $\left(\mathcal{M}_{n}, \mathcal{T}_{l}\right)$ as a (multidimensional) Bernoulli scheme with a continuum of symbols $\omega \subset[-1,1]$.

Our approach to the study of the dynamics generated by the group $\left(\mathcal{B}(\sigma), T_{h}\right)$ is based on the following elementary observation: according to the classical Kotel'nikov formula (see, for example, [7] or [15]), any function $w \in \mathbb{B}_{\sigma}\left(\mathbb{R}^{1}\right) \cap L^{2}\left(\mathbb{R}^{1}\right)$ can be uniquely recovered from its values on the lattice $\rho \mathbb{Z}, \rho:=\frac{\pi}{\sigma}$ :

$$
w(x)=\sum_{l=-\infty}^{\infty} w(\rho l) \frac{\sin (\sigma x-\pi l)}{\sigma x-\pi l}, \quad w \in \mathbb{B}_{\sigma}\left(\mathbb{R}^{1}\right) \cap L^{2}\left(\mathbb{R}^{1}\right) .
$$

(See also the Kotel'nikov-Cartwright formula in [15] for example, for the recovery of an arbitrary function $w \in \mathbb{B}_{\sigma}$.) Given an arbitrary sequence $v=\left\{v_{l}\right\}_{l=-\infty}^{\infty} \in l_{2},(8.3)$ allows 
us to construct a function $w \in \mathbb{B}_{\sigma} \cap L^{2}$ such that $w(\rho l)=v_{l}$ for any $l \in \mathbb{Z}$. Moreover, the spatial shift $T_{\rho} w$ of the function $w$ obviously corresponds to the shift $\mathcal{T}_{1} v$ of the sequence $v$. This leads to a description of the dynamics of spatial translations on $\mathbb{B}_{\sigma} \cap L^{2}$ in terms of the Bernoulli schemes introduced in Definition 8.1 (with the additional restriction $\{v(l)\}_{l=-\infty}^{\infty} \in l^{2}$ ). Generalizing the representation (8.3) to the case of functions in $\mathbb{B}_{\sigma}$ (in the spirit of the Kotel'nikov-Cartwright formula) we obtain the following result.

Theorem 8.1. For any $\sigma>0$ there exists a positive number $\rho$ and a mapping

$$
\mathbb{U}: \mathcal{M}_{n} \rightarrow \mathcal{B}(\sigma) \text { such that } \quad T_{\rho l} \circ \mathbb{U}=\mathbb{U} \circ \mathcal{T}_{l}, \quad l \in \mathbb{Z}^{n} .
$$

Moreover, any function $\phi$ with polynomial growth satisfies the estimates

$$
C_{1}^{-1}\left\|v_{1}-v_{2}\right\|_{L_{\phi}^{\infty}\left(\mathbb{Z}^{n}\right)} \leq\left\|\mathbb{U}\left(v_{1}\right)-\mathbb{U}\left(v_{2}\right)\right\|_{L_{\phi}^{\infty}\left(\mathbb{R}^{n}\right)} \leq C_{1}\left\|v_{1}-v_{2}\right\|_{L_{\phi}^{\infty}\left(\mathbb{Z}^{n}\right)},
$$

where the constant $C_{1}$ depends on the degree $N$ of the polynomial growth of the function $\phi$ and the constant $C_{\phi}$, but is independent of $v_{1}, v_{2} \in \mathcal{M}^{n}$ and the explicit form of the function $\phi$.

Proof. Suppose that $\psi \in \mathbb{B}_{\frac{\sigma}{2}}^{\mathrm{Re}}\left(\mathbb{R}^{1}\right)$ satisfies the conditions

$$
\psi(0)=1, \quad|\psi(x)| \leq C_{N}\left(1+|x|^{2}\right)^{-N} \quad \forall N \in \mathbb{N} .
$$

It is enough to define $\psi$ as the Fourier transform of $\psi_{1}$, where $\psi_{1} \in C_{0}^{\infty}(\mathbb{R})$ is an arbitrary function that satisfies the conditions

$$
\operatorname{supp} \psi_{1} \subset\left[-\frac{\sigma}{2}, \frac{\sigma}{2}\right], \psi_{1}(-\xi)=\psi_{1}(\xi) \text { and } \int_{\mathbb{R}} \psi_{1}(\xi) d \xi=1 .
$$

For any $l \in \mathbb{Z}^{n}$ we define the functions

$$
\phi_{l}(x):=\prod_{i=1}^{n} \phi_{l_{i}}\left(x_{i}\right), \quad \phi_{l_{i}}\left(x_{i}\right):=\frac{\sin \left(\frac{\sigma x_{i}}{2}-\pi l_{i}\right)}{\frac{\sigma x_{i}}{2}-\pi l_{i}} \psi\left(x_{i}-\frac{2 \pi l_{i}}{\sigma}\right) .
$$

Then, since $\psi \in \mathbb{B}_{\frac{\sigma}{2}}$ and $\frac{\sin \left(\frac{\sigma x}{2}\right)}{x} \in \mathbb{B}_{\frac{\sigma}{2}}$, we have $\phi_{l} \in \mathbb{B}_{\sigma}^{\mathrm{Re}}\left(\mathbb{R}^{n}\right)$. In addition we obviously have

$$
\phi_{l}(\rho m)=\delta_{l, m} \quad \forall l, m \in \mathbb{Z}^{n}, \quad \text { and } \quad\left|\phi_{l}(x)\right| \leq C_{N}\left(1+|x-\rho l|^{2}\right)^{-N} \quad \forall N \in \mathbb{N},
$$

where $\rho:=\frac{2 \pi}{\sigma}$. We now define the required operator $\mathbb{U}$ by the formula

$$
(\mathbb{U} v)(x):=L^{-1} \sum_{l \in \mathbb{Z}^{n}} v(l) \phi_{l}(x), \quad v \in \mathcal{M}_{n},
$$

where $L$ is a sufficiently large positive number. Indeed, it follows from (8.8) that for any $N \in \mathbb{N}$

$$
\begin{aligned}
|(\mathbb{U} v)(x)| & \leq L^{-1} C_{N} \sum_{l \in \mathbb{Z}^{n}}|v(l)|\left(1+|x-\rho l|^{2}\right)^{-N} \\
& \leq L^{-1} C(N, \rho) \sup _{l \in \mathbb{Z}^{n}}\left\{|v(l)|\left(1+|x-l|^{2}\right)^{-N+n+1}\right\} .
\end{aligned}
$$

This implies both the convergence of the series (8.9) and the left-hand inequality in (8.5). In particular, taking $\phi \equiv 1$ in (8.10), we see that $\left\|\mathbb{U}\left(\mathcal{M}_{n}\right)\right\|_{L^{\infty}\left(\mathbb{R}^{n}\right)} \leq C L^{-1}$. Hence, since $\phi_{l} \in \mathbb{B}_{\sigma}^{\mathrm{Re}}$, the image $\mathbb{U}\left(\mathcal{M}_{n}\right)$ is contained in $\mathcal{B}(\sigma)$ for sufficiently large $L$. The commutation relation (8.4) is an immediate consequence of the definition of the 
operator $\mathbb{U}$ in (8.9). Thus, it remains to verify the left-hand inequality in (8.5). We first observe that $(\mathbb{U} v)(\rho l)=v(l)$ for any $l \in \mathbb{Z}^{n}$. Consequently,

$$
\begin{aligned}
\left|v_{1}(l)-v_{2}(l)\right| & =\left|\mathbb{U}\left(v_{1}\right)(\rho l)-\mathbb{U}\left(v_{2}\right)(\rho l)\right| \\
& \leq C_{N} \sup _{x \in \mathbb{R}^{n}}\left\{\left|\mathbb{U}\left(v_{1}\right)(x)-\mathbb{U}\left(v_{2}\right)(x)\right|\left(1+|x-l|^{2}\right)^{-N}\right\},
\end{aligned}
$$

where the constant $C_{N}$ depends on $N$, but does not depend on $l$. It follows from this estimate and Proposition 7.1 that the right-hand inequality in (8.5) is valid for any weight function $\phi$ with polynomial growth. This completes the proof of Theorem 8.1.

Corollary 8.1. Suppose that the hypotheses of Theorem 7.1 hold. Then there exists a positive number $\rho$ and a mapping

$$
\mathcal{U}: \mathcal{M}_{n} \rightarrow \mathcal{A}, \quad T_{\rho l} \circ \mathcal{U}=\mathcal{U} \circ \mathcal{T}_{l} \quad \forall l \in \mathbb{Z}^{n} .
$$

Moreover, this mapping is Lipschitzian in any weighted metric with polynomial weight, that is,

$$
C_{2}^{-1}\left\|v_{1}-v_{2}\right\|_{L_{\phi}^{\infty}\left(\mathbb{Z}^{n}\right)} \leq\left\|\mathcal{U}\left(v_{1}\right)-\mathcal{U}\left(v_{2}\right)\right\|_{L_{\phi}^{\infty}\left(\mathbb{R}^{n}\right)} \leq C_{2}\left\|v_{1}-v_{2}\right\|_{L_{\phi}^{\infty}\left(\mathbb{Z}^{n}\right)},
$$

where the constant $C_{2}$ depends on the degree $N$ of the polynomial growth of the function $\phi$ and the constant $C_{\phi}$, but is independent of $v_{1}, v_{2} \in \mathcal{M}_{n}$ and the explicit form of the function $\phi$.

Indeed, by Corollary 7.3 and Theorem 8.1 we can take $\mathcal{U}$ to be the mapping $\widetilde{V} \circ \mathbb{U}$. Thus, we have constructed a homeomorphic embedding of the Bernoulli scheme $\left(\mathcal{M}_{n}, \mathcal{T}_{l}\right)$ into the group of spatial translations of the attractor. As a consequence of this embedding, first we prove the existence of $u_{0} \in \mathcal{A}$ with strictly positive generalized spatial entropy (see formula (5.19)).

Corollary 8.2. Suppose that the hypotheses of Theorem 7.1 hold. Then there exists $u_{0} \in \mathcal{A}$ with strictly positive generalized spatial entropy, that is,

$$
0<C_{1} \leq \widehat{h}_{s p}\left(u_{0}\right) \leq C_{2}<\infty .
$$

In fact, since $\widehat{h}_{s p}\left(\mathcal{U}\left(\mathcal{M}_{n}\right)\right)>0$, one can take $u_{0}$ to be the image $\mathcal{U}(v)$ of an arbitrary element $v \in \mathcal{M}_{n}$ such that the closure of its $\mathcal{T}_{l}$-orbit coincides with $\mathcal{M}_{n}$ (such elements $v$ clearly exist).

Finally, to conclude this section we show that any finite-dimensional dynamics can be realized up to a homeomorphism as the restriction of the group of spatial translations to a suitable invariant subset of $\mathcal{A}$.

Corollary 8.3. Let $K \subset \mathbb{R}^{N}$ be an arbitrary compact set and suppose that $F_{i}, i=$ $1, \ldots, n$, is a set of pairwise commuting homeomorphisms of $K$, that is,

$$
F_{i}: K \rightarrow K, \quad i=1, \ldots, n, \quad F_{i} \circ F_{j}=F_{j} \circ F_{i}, \quad i, j=1, \ldots, n .
$$

Then there exists a homeomorphic embedding $\tau_{K}: K \rightarrow \mathcal{A}$ and a positive number $\sigma_{N}$ depending only on $N$ such that

$$
T_{\sigma_{N} e_{x_{i}}} \circ \tau_{K}=\tau_{K} \circ F_{i}, \quad i=1, \ldots, n,
$$

where $e_{x_{i}}$ denotes the ith coordinate vector in $\mathbb{R}^{n}$.

Proof. By Corollary 8.1 it is sufficient to construct an embedding $\widetilde{\tau}_{K}$ of the compact set $K$ into the Bernoulli scheme $\left(\mathcal{M}_{n}, \mathcal{T}_{l}\right)$ satisfying the commutation relations (8.15). Moreover, we can assume without loss of generality that the compact set $K$ is a subset of the cube $[-1,1]^{M^{n}}$ for some $M$ (such that $\left.M^{n} \geq N\right)$. We let $\left\{(x)_{i_{1}, \ldots, i_{n}}, i_{k}=\right.$ 
$0, \ldots, M-1\}$ denote the coordinates of a point $x \in \mathbb{R}^{M^{n}}$. Then the required mapping $\widetilde{\tau}_{K}: K \rightarrow \mathcal{M}_{n}$ is defined by the standard formula

$$
\begin{aligned}
\tau(k)(l) & :=\left(F^{\left(a_{1}\right)} \circ \cdots \circ F^{\left(a_{n}\right)}(k)\right)_{b_{1}, \ldots, b_{n}}, \\
l_{i}=a_{i} M+b_{i}, \quad a_{i} & \in \mathbb{Z}, \quad b_{i} \in\{0, \ldots, M-1\}, \quad k \in K,
\end{aligned}
$$

that is, $a_{i}=\left[\frac{l_{i}}{M}\right]$ and $b_{i}$ is the remainder after division of $l_{i}$ by $M$ (henceforth we denote by $F^{(l)}, l \in \mathbb{Z}$, the $l$ th iteration of a homeomorphism $F$ ).

It is easy to verify that the mapping thus defined is a continuous embedding of $K$ into $\mathcal{M}_{n}$ and therefore a homeomorphism of $K$ onto $\widetilde{\tau}_{K}(K)$. Moreover, its definition implies the commutation relations

$$
\mathcal{T}_{M e_{x_{i}}} \circ \widetilde{\tau}_{K}=\widetilde{\tau}_{K} \circ F_{i}, \quad i=1, \ldots, n .
$$

Corollary 8.3 is proved.

Remark 8.1. As in the case of classical dynamical systems, the embedding of the Bernoulli scheme $\left(\mathcal{M}_{n}, \mathcal{T}_{l}\right)$ into the group of spatial translations on the attractor constructed in Corollary 8.1 is related to the existence of homoclinic orbits. Indeed, the function $u_{0}:=\mathcal{U}\left(\delta_{0}\right)$, where $\delta_{0}(l):=\delta_{0 l}$, is an elementary homoclinic orbit to the zero equilibrium. Moreover, it follows from (8.12) that $u_{0}(x)$ is decreasing as $|x| \rightarrow \infty$ faster than any power of $|x|$. However, in contrast to the classical case, we note that (8.9) allows us to "sum" the corresponding shifts of the elementary homoclinic orbit $u_{0}$ not only with coefficients $\omega_{l} \in\{0,1\}$, but with any $\omega_{l} \in[-1,1]$, which gives rise to an embedding of the Bernoulli scheme with infinitely many symbols.

Remark 8.2. It is easy to show (see, for example, [31) that

$$
\operatorname{dim}_{s p}\left(\mathcal{M}_{n}, \mathcal{T}_{l}\right)=1
$$

where $\operatorname{dim}_{s p}$ is defined by (5.22). Thus, from the embedding (8.11) it follows that the topological invariant (5.22) for the action of the group of spatial translations on the attractor is positive:

$$
\operatorname{dim}_{s p}\left(\mathcal{A}, T_{h}\right) \geq C>0
$$

\section{Construction of the auXiliary Spatial Dynamical System}

In the preceding section we constructed a description of the spatial chaos arising on the attractor $\mathcal{A}$ in terms of embeddings of Bernoulli schemes with infinitely many symbols (see Corollary 8.1). Note, however, that by construction the image $\mathcal{U}\left(\mathcal{M}_{n}\right)$ is contained in the strongly unstable manifold $\mathcal{V}_{0}$ of the zero equilibrium of equation (0.1). Therefore, this construction does not give meaningful information on the nature of the temporal dynamics generated by (0.1). In spite of this, it may be possible to give a similar description of the temporal dynamics generated by equation (0.1) based on the construction of a strongly unstable manifold (given in §6) and a certain trick of "interchanging" spatial and temporal directions. Suppose we construct an auxiliary dynamical system $\mathcal{S}_{x_{1}}: \Phi_{b} \rightarrow \Phi_{b}$ such that its attractor coincides (in a sense) with the attractor $\mathcal{A}$ of the original system, the spatial direction $x_{1}$ plays the role of time, and the variables $\left(t, x_{2}, \ldots, x_{n}\right)$ play the role of spatial variables. Then the description of the spatial chaos in this auxiliary dynamical system (analogous to that in $\S 6-8$ ) will give a description of the space-time dynamics in the original system corresponding to the hyperplane $V_{n}:=\operatorname{span}\left\{e_{t}, e_{x_{2}}, \ldots, e_{x_{n}}\right\}$.

The main aim of this section is the construction of a dynamical system with the properties described above. First of all, we can assume without loss of generality that 
S. V. ZELIK

the vector $\vec{L}$ in $(0.1)$ is directed along the first coordinate vector:

$$
\vec{L}=L e_{x_{1}}, \quad L \geq 0 .
$$

Indeed, the general case can be reduced to (9.1) by a suitable orthogonal change of coordinates $x^{\prime}=M x, M \in S O(n)$. We consider the following parabolic boundary-value problem in the domain $\Omega_{x_{1}}:=\left\{t \in \mathbb{R}, x_{1} \in \mathbb{R}_{+}, x_{i} \in \mathbb{R}, i=2, \ldots, n\right\}$ constructed with respect to $(0.1)$ :

$$
\left\{\begin{array}{l}
\partial_{t} u=a \partial_{x_{1}}^{2} u-L \partial_{x_{1}} u+a \Delta_{x^{\prime}} u-\lambda_{0} u-f(u), \\
\left.u\right|_{x_{1}=0}=u^{0},
\end{array}\right.
$$

where $x^{\prime}:=\left(x_{2}, \ldots, x_{n}\right)$ and the boundary condition $u^{0}$ is assumed to belong to the space $\Psi_{b}:=L^{\infty}\left(\mathbb{R}_{t} \times \mathbb{R}_{x^{\prime}}^{n-1}\right)$. The following theorem establishes that this boundaryvalue problem has a unique solution if $L$ is sufficiently large.

Theorem 9.1. Suppose that a function $f(u)$ satisfies (0.1), the vector $\vec{L}$ has the form (9.1) and the following condition holds:

$$
L^{2}-4 a K>0,
$$

where the constant $K$ is the same as in (0.3). Then for any $u^{0} \in \Psi_{b}$, (9.2) has a unique solution $u(t, x)$ that is contained in $L^{\infty}\left(\Omega_{x_{1}}\right)$ and satisfies

$$
\left\|u\left(x_{1}\right)\right\|_{\Psi_{b}} \leq C\left(\left\|u^{0}\right\|_{\Psi_{b}} e^{-\alpha x_{1}}+1\right),
$$

where the positive constants $C$ and $\alpha$ are independent of $u^{0}$. Moreover, any two solutions $u_{1}$ and $u_{2}$ of (9.2) contained in $L^{\infty}\left(\Omega_{x_{1}}\right)$ satisfy

$$
\left\|u_{1}\left(x_{1}\right)-u_{2}\left(x_{1}\right)\right\|_{\Psi_{b}} \leq C e^{\Lambda_{0} x_{1}}\left\|u_{1}(0)-u_{2}(0)\right\|_{\Psi_{b}},
$$

where $\Lambda_{0}:=\frac{L}{2 a}$.

Proof. First we prove (9.4). As in the proof of Theorem 1.1, we consider the function $v(t, x)=|u(t, x)|^{2}$, which obviously satisfies the differential inequality

$$
\begin{aligned}
\partial_{t} w-a \Delta_{x} w+L \partial_{x_{1}} w+2 \lambda_{0} w & =-2 a\left|\nabla_{x} w(t, x)\right|^{2}-2 f(u) \cdot u \leq 2 C, \\
\left.w\right|_{x_{1}=0} & =\left|u^{0}\right|^{2} .
\end{aligned}
$$

Note that the function $w_{1}(t, x)=w\left(x_{1}\right):=\left\|u^{0}\right\|_{\Psi_{b}}^{2} e^{-\alpha x_{1}}+\lambda_{0}^{-1} C$ satisfies

$$
\partial_{t} w_{1}-a \Delta_{x} w_{1}-L \partial_{x_{1}} w_{1}+2 \lambda_{0} w_{1} \geq 2 C
$$

if the constant $\alpha>0$ is sufficiently small. Therefore, by the maximum principle for bounded solutions of parabolic inequalities of the form (9.6) (see, for example, [5]) we obtain the estimate $w(t, x) \leq w_{1}(t, x)$, which proves (9.4) The existence of a solution of (9.2) follows in a standard way from (9.4) see [5]. Thus, it remains to verify (9.5).

Let $u_{1}$ and $u_{2}$ be two bounded solutions of (9.2). Consider the function $v(t, x):=$ $e^{-\Lambda_{0} x_{1}}\left(u_{1}(t, x)-u_{2}(t, x)\right)$. Clearly, it satisfies

$$
\partial_{t} v-a \Delta_{x} v+\lambda_{0} v+\left(L \Lambda_{0}-a \Lambda_{0}^{2}+l(t, x)\right) v=0,\left.\quad w\right|_{x_{1}=0}=u_{1}(0)-u_{2}(0),
$$

where

$$
l(t, x):=\int_{0}^{1} f^{\prime}\left(s u_{1}(t, x)+(1-s) u_{2}(t, x)\right) d s .
$$

According to conditions (0.1) and (9.3) we have

$$
L \Lambda_{0}-a \Lambda_{0}^{2}+l(t, x) \geq L \Lambda_{0}-a \Lambda_{0}^{2}+K>0,
$$


and therefore $w(t, x)=|v(t, x)|^{2}$ satisfies a differential inequality of the form

$$
\partial_{t} w-a \Delta_{x} w+2 \lambda_{0} w \leq 0,\left.\quad w\right|_{x_{1}=0}=\left|u_{1}(0)-u_{2}(0)\right|^{2} .
$$

Applying the maximum principle to (9.9) (recall that $\Lambda_{0}>0$ and therefore $w \in L^{\infty}\left(\Omega_{x_{1}}\right)$ ) we obtain (9.5). Theorem 9.1 is proved.

Corollary 9.1. Suppose that the hypotheses of Theorem 9.1 hold. Then problem (9.2) generates the Lipschitz semigroup

$$
\mathcal{S}_{x_{1}}: \Psi_{b} \rightarrow \Psi_{b}, \quad \mathcal{S}_{x_{1}} u^{0}:=u\left(x_{1}\right), \quad \text { where } u\left(x_{1}\right) \text { is a solution of }(9.2) .
$$

The following assertion shows that the semigroup (9.10) is Lipschitzian not only in $\Psi_{b}$, but also in the weighted spaces $\Psi_{\phi}$, where $\phi$ is a weight function with a sufficiently small exponential growth rate.

Corollary 9.2. Suppose that the hypotheses of Theorem 9.1 hold. Then for any weight function $\phi$ with a sufficiently small exponential growth rate $\alpha \leq \varepsilon_{0}$

$$
\left\|\mathcal{S}_{x_{1}} u^{0}-\mathcal{S}_{x_{1}} u_{1}^{0}\right\|_{\Psi_{\phi}} \leq C e^{\Lambda_{0} x_{1}}\left\|u^{0}-u_{1}^{0}\right\|_{\Psi_{\phi}},
$$

where the constant $C$ depends on $C_{\phi}$ but is independent of the form of the weight function $\phi$.

Arguing similarly to the proof of (9.5) and applying the trick of multiplying by the weight functions (1.10) (described in the proof of Theorem 1.2), to inequality (9.9) we can easily obtain estimate (9.11). (Instead of this trick one can use the explicit estimates for the Green's function of the problem (9.9) which is exponentially decreasing at infinity due to the presence of the summand $\lambda_{0} w$ with $\lambda_{0}>0$; see [5].)

Our next aim is to prove that the semigroup (9.10) is differentiable with respect to the "initial conditions" and to obtain an analogue of Corollary 1.4. First we need to consider the "variational equation" corresponding to problem $(9.2)$. Let $u\left(x_{1}\right):=\mathcal{S}_{x_{1}} u^{0}$ be an arbitrary trajectory of the semigroup (9.10). Consider the following inhomogeneous analogue of the linearization of equation (9.2) along this trajectory:

$$
\partial_{t} v-a \Delta_{x} v+L \partial_{x_{1}} v+\lambda_{0} v+f^{\prime}(u(t, x)) v=h(t, x),\left.\quad v\right|_{x_{1}=0}=v^{0},
$$

for some external force $h(t, x)$. The following proposition shows that (9.12) is uniquely solvable in the corresponding space of functionals.

Proposition 9.1. Suppose that the hypotheses of Theorem 9.1 hold and let

$$
e^{-\Lambda_{0} x_{1}} h \in L^{\infty}\left(\Omega_{x_{1}}\right), \quad \Lambda_{0}:=\frac{L}{2 a} .
$$

Then (9.12) has a unique solution in the class

$$
e^{-\Lambda_{0} x_{1}} v \in L^{\infty}\left(\Omega_{x_{1}}\right)
$$

and for any weight function $\phi$ with exponential growth rate $\alpha \leq \varepsilon_{0}$

$$
\left\|v\left(x_{1}\right)\right\|_{\Psi_{\phi}} \leq C e^{-\left(\Lambda_{0}-\beta\right) x_{1}}\left\|v^{0}\right\|_{\Psi_{\phi}}+\sup _{y \in \mathbb{R}_{+}}\left\{e^{\Lambda_{0}\left(x_{1}-y\right)-\beta\left|x_{1}-y\right|}\|h(y)\|_{\Psi_{\phi}}\right\}
$$

where the positive constants $C$ and $\beta$ are independent of $u^{0}$ and the form of the weight function $\phi$.

Proof. In a similar way to the proof of Theorem 9.1, the function

$$
w(t, x):=e^{-2 \Lambda_{0} x_{1}}|v(t, x)|^{2}
$$

satisfies the inequality

$$
\partial_{t} w-a \Delta_{x} w+\lambda_{0} w \leq C|\widetilde{h}(t, x)|^{2}, \quad \widetilde{h}:=e^{-\Lambda_{0} x_{1}} h(t, x) .
$$


Using the maximum principle and the trick of multiplying equation (9.16) by the weight function $e^{-\varepsilon \sqrt{1+\left|(t, x)-\left(t_{0}, x_{0}\right)\right|^{2}}}$ for $\varepsilon>0$ sufficiently small, as in the proof of Theorem 1.2 we can easily obtain the estimate

$$
\begin{aligned}
w(t, x) \leq & C e^{-\varepsilon x_{1}} \sup _{\left(t_{0}, x_{0}^{\prime}\right) \in \mathbb{R}^{n}}\left\{e^{-\varepsilon\left|\left(t, x^{\prime}\right)-\left(t_{0}, x_{0}^{\prime}\right)\right|}\left|v^{0}\left(t_{0}, x_{0}^{\prime}\right)\right|^{2}\right\} \\
& +\sup _{\left(t_{0}, x_{0}\right) \in \Omega_{x_{1}}}\left\{e^{-\varepsilon\left|(t, x)-\left(t_{0}, x_{0}\right)\right|}|\widetilde{h}(t, x)|^{2}\right\} .
\end{aligned}
$$

Together, (9.17) and (1.28) yield (9.15) for any weight function with exponential growth rate $\alpha<\frac{\varepsilon}{2}$, which proves Proposition 9.2. (As in the proof of Corollary 9.2, (9.17) is an immediate consequence of the fact that the Green's function of equation (9.16) decays exponentially at infinity.)

We are now ready to verify that the semigroup (9.1) is differentiable.

Theorem 9.2. Suppose that the hypotheses of Theorem 9.1 hold. Then the semigroup (9.10) generated by problem (9.2) belongs to the class $C^{1, \alpha}\left(\Psi_{b}, \Psi_{b}\right)$ for some $0<\alpha \leq 1$, and its Fréchet derivative is calculated by the formula $D_{u^{0}} \mathcal{S}_{x_{1}}\left(u_{0}\right) \xi:=v_{\xi}\left(x_{1}\right)$ where $v_{\xi}\left(x_{1}\right)$ is the unique solution of problem (9.12) with $h=0$ and $v^{0}=\xi$, which was constructed in Proposition 9.1. Moreover, for any weight function $\phi$ with a sufficiently small exponential growth rate and any $u^{0}, u_{1}^{0} \in \Psi_{b} \cap \Psi_{\phi}$

$$
\begin{aligned}
\| \mathcal{S}_{x_{1}}\left(u^{0}\right)-\mathcal{S}_{x_{1}}\left(u_{1}^{0}\right)- & D_{u^{0}} \mathcal{S}_{x_{1}}\left(u^{0}\right)\left(u^{0}-u_{1}^{0}\right) \|_{\Psi_{\phi}} \\
\leq & \leq e^{\Lambda_{0} t}\left\|u^{0}-u_{1}^{0}\right\|_{\Psi_{b}}^{\alpha} \cdot\left\|u^{0}-u_{1}^{0}\right\|_{\Psi_{\phi}} .
\end{aligned}
$$

The constant $C$ depends only on the $\Psi_{b}$-norms of the initial conditions $u_{0}$ and $u_{1}$ and the constant $C_{\phi}$ in inequality (1.24). (It does not depend on the form of the weight function $\phi$.

Proof. Let $u(t, x)$ and $u_{1}(t, x)$ be the solutions of (9.2) corresponding to the boundary conditions $u^{0}$ and $u_{1}^{0}$ respectively. Let $v(t, x)$ be the solution of $(9.12)$ with $h=0$ and $v^{0}=u^{0}-u_{1}^{0}$. Then the function $w(t, x):=u(t, x)-u_{1}(t, x)-v(t, x)$ clearly satisfies

$$
\partial_{t} w-a \Delta_{x} w+L \partial_{x_{1}} w+\lambda_{0} w+f^{\prime}(u(t, x)) w=h_{u, u_{1}}(t, x),\left.\quad w\right|_{x_{1}=0}=0,
$$

where

$$
h_{u, u_{1}}(t, x):=\int_{0}^{1}\left[f^{\prime}\left(u(t, x)-s\left(u(t, x)-u_{1}(t, x)\right)\right)-f^{\prime}(u(t, x))\right] d s \cdot v(t, x) .
$$

We also observe that (9.15) implies that

$$
\left\|v\left(x_{1}\right)\right\|_{\Psi_{\phi}} \leq C e^{\left(\Lambda_{0}-\beta\right) x_{1}}\left\|u^{0}-u_{1}^{0}\right\|_{\Psi_{\phi}}
$$

for some positive $\beta$ independent of $\phi, u^{0}$, and $u_{1}^{0}$. Thus, since $f \in C^{2}\left(\mathbb{R}^{k}, \mathbb{R}^{k}\right)$, using (9.5) and (9.20), for any $0<\alpha \leq 1$ we have

$$
\left\|h_{u, u_{1}}\left(x_{1}\right)\right\|_{\Psi_{\phi}} \leq C_{\alpha} e^{\left(\alpha \Lambda_{0}+\Lambda_{0}-\beta\right) x_{1}}\left\|u^{0}-u_{1}^{0}\right\|_{\Psi_{b}}^{\alpha} \cdot\left\|u^{0}-u_{1}^{0}\right\|_{\Psi_{\phi}},
$$

where the constant $C_{\alpha}$ depends on $\left\|u_{i}^{0}\right\|_{\Psi_{b}}, \alpha$, and $C_{\phi}$, but does not depend on the explicit form of $\phi$. Fixing $\alpha:=\beta \Lambda_{0}^{-1}$ we now obtain that the function $h_{u, u_{1}}$ satisfies (9.13). Applying (9.15) to equation (9.19) and using inequality (9.21) we obtain (9.18). The other assertions of the theorem are immediate consequences of this estimate. Theorem 9.2 is proved. 
To conclude this section we construct the attractor $\mathcal{A}_{s p}$ of the semigroup (9.10) and prove a natural formula connecting this attractor with the attractor $\mathcal{A}$ of the original equation (0.1).

Theorem 9.3. Suppose that the hypotheses of Theorem 9.1 hold. Then the semigroup (9.10) has a locally compact attractor $\mathcal{A}_{\text {sp }}$ in phase space $\Psi_{b}$ (see Definition 2.1), which admits the following description:

$$
\mathcal{A}_{s p}=\left.\mathcal{K}\right|_{x_{1}=0},
$$

where $\mathcal{K}$ is the set of all bounded solutions of (0.1) defined in Theorem 2.1. Thus, the attractors $\mathcal{A}$ and $\mathcal{A}_{\text {sp }}$ are connected by the relation

$$
\mathcal{A}=\left.\mathcal{K}\right|_{t=0}, \quad \mathcal{A}_{s p}=\left.\mathcal{K}\right|_{x_{1}=0} .
$$

Moreover, the attractor $\mathcal{A}_{s p}$ is invariant under the group $\widetilde{T}_{h}$ of "spatial" translations:

$$
\widetilde{T}_{h} \mathcal{A}_{s p}=\mathcal{A}_{s p}, \quad\left(\widetilde{T}_{h}\right) u^{0}\left(t, x^{\prime}\right):=u\left(t+h_{1}, x^{\prime}+h^{\prime}\right), \quad h \in \mathbb{R}^{n} .
$$

We can derive the existence of the attractor $\mathcal{A}_{s p}$ from the dissipative estimate (9.4) and the interior smoothing estimate for the parabolic problem (9.2) in the same way as in the proof of Theorem 2.1. Moreover, since the equations (0.1) and (9.2) coincide, the sets of their bounded solutions in $\mathbb{R}^{n+1}$ also coincide. This gives both (9.22) and (9.23). Since (9.2) is invariant under the group $\left\{\widetilde{T}_{h}, h \in \mathbb{R}^{n}\right\}$, (9.24) follows immediately.

Remark 9.1. A method of constructing an auxiliary spatial dynamical system, similar to the one discussed above, has been used by many authors for studying elliptic boundaryvalue problems in cylindrical domains by the methods of the theory of dynamical systems (see [2, 17, 29] and the references therein). As the results of this section show, this method can also be applied in the case of parabolic boundary-value problems.

\section{Space-time chaos in Reaction-Diffusion equations in $\mathbb{R}^{n}$}

In this section we construct a strongly unstable manifold for the zero equilibrium of the spatial dynamical system (9.10) that is diffeomorphic to the space $\mathbb{B}_{\sigma}^{\mathrm{Re}}$. Moreover, as in $\S 8$, we use this to derive a description of the space-time dynamics, corresponding to the hyperplane $V_{n}:=\operatorname{span}\left\{e_{t}, e_{x_{2}}, \ldots, e_{x_{n}}\right\}$, on the attractor $\mathcal{A}$ of the original equation (0.1). We begin by stating an analogue of Theorem 8.1 for the spatial dynamical system (9.10).

Theorem 10.1. Suppose that the hypotheses of Theorem 9.1 hold and suppose in addition that

$$
f(0)=0, \quad\left[f^{\prime}(0)\right]^{*}=f^{\prime}(0), \quad \text { and } \quad \sigma\left(-f^{\prime}(0)-\lambda_{0}, \mathbb{R}^{k}\right) \cap\{\operatorname{Re} \lambda>0\} \neq \varnothing .
$$

Then there exist positive numbers $\sigma$ and $\rho_{0}$, a vector $\vec{e} \in \mathbb{R}^{k}$, a subspace

$$
\Psi_{+}=\Psi_{+}(\sigma):=\mathbb{B}_{\sigma}^{\mathrm{Re}} \cdot \vec{e},
$$

and a smooth $\left(C^{1, \alpha}\right.$-smooth $)$ mapping

$$
\overline{\mathbb{V}}_{0}: B\left(\rho_{0}, 0, \Psi_{+}\right) \rightarrow \mathcal{A}_{s p} \quad \text { such that } \quad \widetilde{T}_{h} \circ \overline{\mathbb{V}}_{0}=\overline{\mathbb{V}}_{0} \circ \widetilde{T}_{h} \quad \forall h \in \mathbb{R}^{n},
$$

and

$$
\left\|\overline{\mathbb{V}}_{0} v-v\right\|_{\Psi_{b}} \leq C\|v\|_{\Psi_{b}}^{1+\alpha} \quad \forall v \in B\left(\rho_{0}, 0, \Psi_{+}\right),
$$

where $\alpha>0$ is defined in Theorem 9.2. Moreover, for any weight function $\phi$ of polynomial growth the following estimates hold:

$$
C_{1}^{-1}\left\|v_{1}-v_{2}\right\|_{\Psi_{\phi}} \leq\left\|\overline{\mathbb{V}}_{0}\left(v_{1}\right)-\overline{\mathbb{V}}_{0}\left(v_{2}\right)\right\|_{\Psi_{\phi}} \leq C_{1}\left\|v_{1}-v_{2}\right\|_{\Psi_{\phi}}
$$


for any $v_{1}, v_{2} \in B\left(\rho_{0}, 0, \Psi_{+}\right)$. The constant $C_{1}$ depends only on the constants $N$ and $C_{\phi}$ in (7.2) and does not depend on the explicit form of the weight function $\phi$.

Proof. As in the proof of Theorem 7.1, we wish to construct a strongly unstable manifold of the zero equilibrium of the solution operator $S:=\mathcal{S}_{1}: \Psi_{b} \rightarrow \Psi_{b}$ for equation (9.2) per unit time. To do this, we need to verify that $S$ satisfies the hypotheses of Theorem 6.1 and Corollary 6.5. Now, in this case $\Phi:=\Psi_{b}, S_{0}:=D_{u^{0}} \mathcal{S}_{1}(0)$, and $\mathcal{B}(u):=S(u)-S_{0}(u)$, where $D_{u^{0}} \mathcal{S}_{x_{1}}(0)$ is the solution operator for the linear parabolic problem

$$
\partial_{t} v=a \Delta_{x} v-L \partial_{x_{1}} v-f^{\prime}(0) v-\lambda_{0} v,\left.\quad v\right|_{x_{1}=0}=v^{0}, \quad e^{-\Lambda_{0} x_{1}} v \in L^{\infty}\left(\Omega_{x_{1}}\right) .
$$

By Theorem 9.2, this is the linearization of (9.2) along the zero solution. Thus it follows from Theorem 9.2 that $\mathcal{B} \in C^{1, \alpha}\left(\Psi_{b}, \Psi_{b}\right), \mathcal{B}(0)=\mathcal{B}^{\prime}(0)=0$, and

$$
\left\|\mathcal{B}(u)-\mathcal{B}\left(u_{1}\right)\right\|_{\Psi_{\phi}} \leq C\left(\|u\|_{\Psi_{b}}^{\alpha}+\left\|u_{1}\right\|_{\Psi_{b}}^{\alpha}\right)\left\|u-u_{1}\right\|_{\Psi_{\phi}}
$$

where $u, u_{1} \in \Psi_{b}, \phi$ is an arbitrary weight function with a sufficiently small exponential growth rate, and the constant $C$ depends on the $\Psi_{b}$-norms of $u, u_{1}$, and $C_{\phi}$, but does not depend on the explicit form of the function $\phi$. Thus, the nonlinear function $\mathcal{B}(u)$ satisfies all the hypotheses of Theorem 6.1 and Corollary 6.5. It remains to verify the conditions on the linear operator $S_{0}$. For that we shall need the following lemmas.

Lemma 10.1. Suppose that the hypotheses of Theorem 10.1 hold. Then the spectral radius of the operator $S_{0}$ is calculated by the formula

$$
r_{0}\left(S_{0}\right)=\exp \left\{\frac{L-\sqrt{L^{2}-4 a \max \sigma\left(-f^{\prime}(0)-\lambda_{0}, \mathbb{R}^{k}\right)}}{2 a}\right\} .
$$

In particular, there exists an eigenvector $\vec{e} \in \mathbb{R}^{k}$ of the operator $-f^{\prime}(0)-\lambda_{0}$ such that

$$
r_{0}\left(S_{0}\right)=\exp \left\{\frac{L-\sqrt{L^{2}-4 a \bar{\Lambda}_{0}}}{2 a}\right\}, \quad\left(-f^{\prime}(0)-\lambda_{0}\right) \vec{e}=\bar{\Lambda}_{0} \vec{e} .
$$

Moreover, if $\phi$ is a weight function with exponential rate of growth $\alpha>0$ and $\widetilde{S}_{0}$ is an extension of the operator $S_{0}$ to a continuous operator in $\Psi_{\phi}$, then

$$
r_{0}\left(\widetilde{S}_{0}, \Phi_{\phi}\right) \leq r_{0}\left(S_{0}\right)+C \alpha,
$$

where the constant $C$ is independent of $\alpha, \phi$, and $C_{\phi}$.

Proof. If $\vec{e}$ is an eigenvector of $-f^{\prime}(0)-\lambda_{0}$ corresponding to the eigenvalue $\bar{\Lambda}_{0}$, the function

$$
u_{\bar{\Lambda}_{0}}\left(x_{0}\right)=e^{\Lambda\left(\bar{\Lambda}_{0}\right) x_{1}} \vec{e}, \quad \Lambda\left(\bar{\Lambda}_{0}\right):=\frac{L-\sqrt{L^{2}-4 a \bar{\Lambda}_{0}}}{2 a}
$$

satisfies equation (10.6). Also, by $(9.3), \Lambda\left(\bar{\Lambda}_{0}\right) \leq \frac{L}{2 a}$ and so

$$
r_{0}\left(S_{0}\right) \geq e^{\Lambda\left(\max \sigma\left(-f^{\prime}(0)-\lambda_{0}, \mathbb{R}^{k}\right)\right)} .
$$

To prove the reverse inequality we observe that, since the operator $f^{\prime}(0)$ is selfadjoint, its spectrum is real and

$$
\left(-f^{\prime}(0)-\lambda_{0}\right) v \cdot v \leq \bar{\Lambda}_{0} v \cdot v,
$$

where $\bar{\Lambda}_{0}$ is the maximum eigenvalue of the operator $-f^{\prime}(0)-\lambda_{0}$. Hence, as in the proof of Theorem 9.1, if $v(t, x)$ is an arbitrary solution of equation (10.6) and $\theta \in \mathbb{R}$ is an arbitrary number, the function $w(t, x):=e^{-2 \theta x_{1}}|v(t, x)|^{2}$ satisfies the inequality

$$
\partial_{t} w-a \Delta_{x} w+(L-2 a \theta) \partial_{x_{1}} w+2\left(L \theta-a \theta^{2}-\bar{\Lambda}_{0}\right) w \leq 0 .
$$


In order to apply the maximum principle to $(10.14)$ we require that $L \theta-a \theta^{2}-\bar{\Lambda}_{0} \geq 0$. Note that $\theta=\Lambda\left(\bar{\Lambda}_{0}\right)$ is the smallest value of $\theta$ that satisfies this condition. Hence the maximum principle implies the a priori estimate

$$
\left\|v\left(x_{1}\right)\right\|_{\Psi_{b}} \leq e^{\Lambda\left(\bar{\Lambda}_{0}\right) x_{1}}\left\|v^{0}\right\|_{\Psi_{b}}
$$

for solutions $v(t, x)$ of problem (10.6) that satisfy $e^{-\Lambda\left(\bar{\Lambda}_{0}\right) x_{1}} v \in L^{\infty}\left(\Omega_{x_{1}}\right)$. Using the a priori estimate (10.15) the proof that there exists a solution in this class is standard (see, for example, [5]). Since $\Lambda\left(\bar{\Lambda}_{0}\right) \leq \frac{L}{2 a}$, Proposition 9.1 implies that $D_{u^{0}} \mathcal{S}_{x_{1}}(0) v^{0}=$ $v\left(x_{1}\right)$. It remains to observe that (10.15) implies the reverse inequality to (10.12). Thus, (10.8) is proved. As in Lemma 7.1, (10.9) is an immediate consequence of (10.8), and (10.10) can be proved in the same way as in Lemma 7.2. Lemma 10.1 is proved.

Now, applying (10.1) and (10.8) we have $r_{0}\left(S_{0}\right)>1$. Condition (6.2) is verified.

The following lemma is an analogue of Lemma 7.3 for the case of the spatial dynamical system (9.10).

Lemma 10.2. There exists $\theta_{0}>1$ satisfying (6.6), $\sigma>0$, and a subspace $\Psi_{+}$that is invariant (under $S_{0}$ and $\widetilde{T}_{h}$ ) and is defined by (10.2), where the vector $\vec{e}$ satisfies (10.9), which are such that the restriction $S_{+}$of the operator $S_{0}$ to the invariant subspace $\Psi_{+}$is invertible and satisfies (6.5). Moreover, for any $N>0$ there exists $\varepsilon_{N}>0$ such that

$$
\left\|S_{+}^{-l} v_{+}\right\|_{\Psi_{\psi_{\varepsilon, N, x_{0}}}} \leq C \theta_{0}^{-l}\left\|v_{+}\right\|_{\Psi_{\phi_{\varepsilon}, N, x_{0}}} \quad \forall v_{+} \in \Psi_{+}, \quad l \in \mathbb{N}
$$

where $\psi_{\varepsilon, N, x_{0}}(x):=\left(1+\varepsilon\left|x-x_{0}\right|\right)^{-N}, 0<\varepsilon \leq \varepsilon_{N}$, and the constant $C$ is independent of $N, \varepsilon \leq \varepsilon_{N}$, and $x_{0} \in \mathbb{R}^{n}$.

The proof of Lemma 10.2 is very similar to the proof of Lemma 7.3; we only need to replace (7.21) by

$$
\widehat{S_{0}^{l} v_{+}}(\xi)=\exp \left\{\begin{array}{c}
l \frac{L-\sqrt{L^{2}+4 a^{2}\left|\xi^{\prime}\right|^{2}-4 a \bar{\Lambda}_{0}+4 i a \xi_{1}}}{2 a} \\
v_{+} \in \Psi_{+}, \quad l \in \mathbb{Z}
\end{array} \widehat{v}_{+}(\xi) \vec{e}\right.
$$

To prove this formula it is sufficient to apply the Fourier $\left(t, x^{\prime}\right)$-transformation to equation (10.6) and use the fact that $\vec{e}$ is an eigenvector of the operator $-f^{\prime}(0)-\lambda_{0}$ with eigenvalue $\bar{\Lambda}_{0}$. Note also that by $(10.8)$ we have

$$
\begin{aligned}
\Sigma(0) & =r_{0}\left(S_{0}\right)>1, \quad \text { where } \\
\Sigma(\xi): & =\exp \left\{\frac{L-\sqrt{L^{2}+4 a^{2}\left|\xi^{\prime}\right|^{2}-4 a \bar{\Lambda}_{0}+4 i a \xi_{1}}}{2 a}\right\} .
\end{aligned}
$$

Therefore, as in the proof of Lemma 7.3, it follows from (10.17) and (10.18) that for sufficiently small $\sigma>0,(10.16)$ holds for some $\theta_{0}$ satisfying (6.6). This proves Lemma 10.2. Moreover, Theorem 10.1 can be derived from Lemmas 10.1-10.2 in the same way as in the proof of Theorem 7.1. Theorem 10.1 is proved.

Remark 10.1. We introduced the technical condition that the matrix $f^{\prime}(0)$ be selfadjoint to ensure that the "maximally unstable" eigenvalue $\bar{\Lambda}_{0}$ of the matrix $-f^{\prime}(0)-\lambda_{0}$ is real. Indeed, if $\operatorname{Im} \bar{\Lambda}_{0} \neq 0$, then the "maximal instability" in equation (10.6) is no longer attained at a spatially homogeneous solution of the form $u\left(x_{1}\right):=r_{0}\left(S_{0}\right)^{x_{1}} \vec{e}$ (as in Lemma 10.1), but at a spatially periodic solution of the form $u\left(x_{1}, t\right)=r_{0}\left(S_{0}\right)^{x_{1}} e^{i \operatorname{Im} \bar{\Lambda}_{0} t} \vec{e}$. In this case the space $\mathbb{B}_{\sigma}$ in (10.2) must be replaced by the more complicated space $\mathbb{B}_{\sigma, \operatorname{Im} \bar{\Lambda}_{0}}$, which consists of the functions whose Fourier transforms have their support 
contained in the shifted cube $\operatorname{Im} \bar{\Lambda}_{0}+[-\sigma, \sigma]^{n}$. This leads to more cumbersome formulae, but these do not change the general scheme of exposition; see [43, 44] .

We now state the analogues of Corollaries $7.1-7.3$ for the spatial dynamical system (9.10).

Corollary 10.1. Suppose that the hypotheses of Theorem 10.1 hold. Then the $\varepsilon$-entropy of the attractor $\mathcal{A}_{s p}$ of (9.10) admits the following lower bound:

$$
\mathbb{H}_{\varepsilon}\left(\left.\mathcal{A}_{s p}\right|_{B_{0}^{R}}, L^{\infty}\left(B_{0}^{R}\right)\right) \geq C_{1} R^{n} \ln _{+} \frac{R_{0}^{\prime}}{\varepsilon},
$$

where the constants $C$ and $R_{0}^{\prime}$ are independent of $R \geq 1$ and $\varepsilon>0$. In particular, the spatial (generalized) topological entropy of the action of the group $\widetilde{T}_{h}$ on the attractor $\mathcal{A}_{s p}$ is strictly positive:

$$
h_{s p}\left(\mathcal{A}_{s p}, \widetilde{T}_{h}\right) \geq C_{1}>0 .
$$

The proof of (10.19) and (10.20) repeats word for word the proof of Corollaries 7.1 and 7.2 and is therefore omitted.

Corollary 10.2. Suppose that the hypotheses of Theorem 10.1 hold. Then there exists a mapping

$$
\overline{\mathbb{V}}: \mathcal{B}(\sigma) \rightarrow \mathcal{A}_{s p}, \quad \mathcal{B}(\sigma):=B\left(1,0, \mathbb{B}_{\sigma}^{\mathrm{Re}}\right),
$$

commuting with the group $\left\{\widetilde{T}_{h}, h \in \mathbb{R}^{n}\right\}$ of "spatial" translations and satisfying the estimate

$$
C_{1}^{-1}\left\|v_{1}-v_{2}\right\|_{L_{\phi}^{\infty}\left(\mathbb{R}^{n}\right)} \leq\left\|\overline{\mathbb{V}}\left(v_{1}\right)-\overline{\mathbb{V}}\left(v_{2}\right)\right\|_{L_{\phi}^{\infty}\left(\mathbb{R}^{n}\right)} \leq C_{1}\left\|v_{1}-v_{2}\right\|_{L_{\phi}^{\infty}\left(\mathbb{R}^{n}\right)}
$$

for any $v_{1}, v_{2} \in \mathcal{B}(\sigma)$, where the constant $C_{1}$ depends only on the constants $N$ and $C_{\phi}$ in inequality (7.3) and does not depend on the explicit form of the weight function $\phi$ with polynomial growth.

In fact, the required mapping is defined by the formula $\overline{\mathbb{V}}(v):=\overline{\mathbb{V}}_{0}\left(\rho^{-1} v \cdot \vec{e}\right)$, where $\overline{\mathbb{V}}_{0}$ is the mapping constructed in Theorem 10.1.

In the following theorem we reformulate the results obtained above in terms of the original dynamical system generated by equation (0.1).

Theorem 10.2. Suppose that the hypotheses of Theorem 10.1 hold. Then the generalized topological entropy of the action of the multiparameter semigroup (1.6) on the attractor $\mathcal{A}$ of equation (0.1) corresponding to the hyperplane $V_{n}:=\operatorname{span}\left\{e_{t}, e_{x_{2}}, \ldots, e_{x_{n}}\right\}$ is strictly positive:

$$
\widehat{h}_{\mathrm{top}}^{1}\left(\mathcal{A}, \mathbb{S}_{(t, h)}^{V_{n}}\right) \geq C_{2}>0
$$

In particular,

$$
\widehat{h}_{\text {top }}^{n}\left(\mathcal{A}, S_{t}\right) \geq C_{3}>0
$$

and therefore

$$
h_{\text {top }}\left(\mathcal{A}, S_{t}\right)=\infty .
$$

Moreover, there exists a positive number $\sigma>0$ and an embedding $\mathbb{W}: \mathcal{B}(\sigma) \rightarrow \mathcal{A}$ that is homeomorphic (in the local topology) such that

$$
\begin{aligned}
S_{t} & \circ \mathbb{W}=\mathbb{W} \circ T_{t e_{x_{1}}}, \quad t \geq 0, \\
T_{h e_{x_{i}}} \circ \mathbb{W} & =\mathbb{W} \circ T_{h e_{x_{i}}}, \quad h \in \mathbb{R}, \quad i=2, \ldots, n .
\end{aligned}
$$


Proof. By (10.20) and Proposition 4.3 we have

$$
\widehat{h}^{1}\left(\mathcal{K}, \mathbb{T}_{(s, h)}^{V_{n}}\right) \geq C \widehat{h}_{s p}\left(\mathcal{A}_{s p}, \widetilde{T}_{h}\right) \geq C C_{1}>0 .
$$

Applying Proposition 4.3 again we obtain (4.24) as required. (Here we have implicitly used the fact that the sets $\mathcal{K}$ of all bounded solutions of equations (0.1) and (9.2) coincide.) Now, (10.24) is an immediate consequence of (10.23) and Theorem 4.1. Thus, it remains to construct an embedding $\mathbb{W}$ that satisfies (10.25). For this we use the embedding (10.21) constructed in Corollary 7.2. First of all, recall that by construction the image $\overline{\mathbb{V}}(\mathcal{B}(\sigma))$ lies on the strongly unstable manifold $\mathcal{V}_{0}$ of the zero equilibrium of the spatial dynamical system (9.10). For any $v_{0} \in \mathcal{V}_{0}$ we let $\Pi_{x_{1}=0}^{-1} v_{0} \in \mathcal{K}$ denote the complete trajectory of the semigroup (9.10) passing through $v_{0}$, that is,

$$
\left(\Pi_{x_{1}=0}^{-1} v_{0}\right)(t, x):= \begin{cases}S_{x_{1}} v_{0} & \text { for } \quad x_{1} \geq 0, \\ S_{x_{1}-\left[x_{1}\right]} \overline{\mathbb{V}}_{\left[x_{1}\right]}\left(\left(\overline{\mathbb{V}}_{0}\right)^{-1}\left(v_{0}\right)\right) & \text { for } \quad x_{1}<0,\end{cases}
$$

where the mappings $\overline{\mathbb{V}}_{0}$ and $\overline{\mathbb{V}}_{n}, n \in \mathbb{Z}_{-}$, are constructed in Theorems 10.1 and 6.1, respectively. Then, by $(6.34),(9.11),(9.15),(10.5)$, and (10.16), for any weight function with polynomial growth and any $v_{1}, v_{2} \in \mathcal{V}_{0}$, we have the estimate

$$
\left\|\Pi_{x_{1}=0}^{-1} v_{1}-\Pi_{x_{1}=0}^{-1} v_{2}\right\|_{L_{e^{-\Lambda_{0}\left|x_{1}\right|}}\left(\mathbb{R}, \Psi_{\phi}\right)} \leq C\left\|v_{1}-v_{2}\right\|_{\Psi_{\phi}}, \quad \Lambda_{0}:=\frac{L}{2 a} .
$$

Hence the mapping $\Pi_{x_{1}=0}^{-1}$ realizes an embedding of the set $\mathcal{V}_{0}$ into the space $\mathcal{K}$ that is homeomorphic (in the local topology).

On the other hand, by Theorem 5.1 the mapping $\Pi_{t=0}$ is a homeomorphism between the sets $\mathcal{A}$ and $\mathcal{K}$. (Indeed, by this theorem the mapping $\Pi_{t=0}$ is one-to-one, and a continuous one-to-one mapping of compact sets is a homeomorphism.)

Thus, we can define the required mapping $\mathbb{W}$ by

$$
\mathbb{W}(v):=\Pi_{t=0} \circ\left(\Pi_{x_{1}=0}\right)^{-1} \circ \overline{\mathbb{V}}(v), \quad v \in \mathcal{B}(\sigma) .
$$

The validity of the commutation relations (10.25) immediately follows from the definition of $\mathbb{W},(10.27)$. Theorem 10.2 is proved.

Thus, we have constructed a homeomorphic embedding of the model dynamics $\left(\mathcal{B}(\sigma), T_{h}\right)$ into the space-time dynamical system $\left(\mathcal{A}, \mathbb{S}_{(t, h)}^{V_{n}}\right)$ generated by $(0.1)$ and the hyperplane $V_{n}:=\operatorname{span}\left\{e_{t}, e_{x_{2}}, \ldots, e_{x_{n}}\right\}$. To conclude this section, we state several corollaries of this embedding analogous to the results of $\S 8$.

Corollary 10.3. Suppose that the hypotheses of Theorem 10.1 hold. Then there exist positive numbers $\sigma$ and $\rho$ and a homeomorphic embedding

$$
\tau: \mathcal{M}_{n} \rightarrow \mathcal{A}
$$

where $\mathcal{M}_{n}:=[-1,1]^{\mathbb{Z}^{n}}$ (see Definition 8.1), such that

$$
S_{\rho l} \circ \tau=\tau \circ \mathcal{T}_{l_{e_{1}}}, \quad T_{\rho l e_{x_{i}}} \circ \tau=\tau \circ \mathcal{T}_{l_{e_{i}}}, \quad i=2, \ldots, n, \quad l \in \mathbb{Z} .
$$

In fact, the required mapping $\tau$ is given by the formula $\tau=\mathbb{W} \circ \mathbb{U}$, where the mappings $\mathbb{U}$ and $\mathbb{W}$ are defined in Theorems 8.1 and 10.2 , respectively.

Corollary 10.4. Suppose that the hypotheses of Theorem 10.1 hold. Let $K \subset \mathbb{R}^{N}$ be an arbitrary compact set in $\mathbb{R}^{n}$ and suppose that $F_{i}, i=1, \ldots, n$, is a set of pairwise commuting homeomorphisms of the compact set $K$, that is,

$$
F_{i}: K \rightarrow K, \quad i=1, \ldots, n, \quad F_{i} \circ F_{j}=F_{j} \circ F_{i}, \quad i, j=1, \ldots, n .
$$


Then there exists a homeomorphic embedding $\widetilde{\tau}_{K}: K \rightarrow \mathcal{A}$ and a positive number $\sigma_{N}$ depending only on $N$ such that

$$
S_{\sigma_{N}} \circ \widetilde{\tau}_{K}=\widetilde{\tau}_{K} \circ F_{1}, \quad T_{\sigma_{N} e_{x_{i}}} \circ \widetilde{\tau}_{K}=\widetilde{\tau}_{K} \circ F_{i}, \quad i=2, \ldots, n,
$$

where $e_{x_{i}}$ denotes the ith coordinate vector in $\mathbb{R}^{n}$.

Now, by Corollary 10.3 it is sufficient to construct an embedding of the finite-dimensional dynamics generated by the homeomorphisms $F_{i}$ into the model dynamical system $\left(\mathcal{M}_{n}, \mathcal{T}_{l}\right)$. Such an embedding was actually constructed in the proof of Corollary 8.3.

The following result shows that when $n>1$, by considering only the temporal part of the dynamics, we can remove the condition that the set $K$ be finite-dimensional.

Corollary 10.5. Suppose that the hypotheses of Theorem 10.1 hold with $n>1$. Suppose further that $K$ is an arbitrary compact metric set, and $F: K \rightarrow K$ is an arbitrary homeomorphism. Then there exists a homeomorphic embedding $\tau_{K}: K \rightarrow \mathcal{A}$ such that

$$
S_{\tau l} \circ \tau_{K}=\tau_{K} \circ F,
$$

where the constant $\rho>0$ is the same as in Corollary 10.3 .

Proof. Just as in the proof of Corollary 10.4, it is sufficient to construct the required embedding into the model dynamical system $\left(\mathcal{M}_{n}, \mathcal{T}_{l_{x_{1}}}\right)$. We now observe that the multidimensional Bernoulli scheme $\mathcal{M}_{n}:=[-1,1]^{\mathbb{Z}^{n}}$ can be interpreted as the one-dimensional Bernoulli scheme with symbol space $[-1,1]^{\mathbb{Z}^{n-1}}$, that is,

$$
\mathcal{M}_{n}=\left([-1,1]^{\mathbb{Z}^{n-1}}\right)^{\mathbb{Z}}
$$

On the other hand, by Uryson's theorem every metric compact set can be homeomorphically embedded into $[-1,1]^{\mathbb{Z}}$ and therefore also into $[-1,1]^{\mathbb{Z}^{n-1}}$ (since $n>1$ ). Now we can construct the required embedding of $K$ into the Bernoulli scheme (10.33) using the standard construction described in the proof of Corollary 8.3. This completes the proof.

Remark 10.2. Corollary 10.5 shows that when $n>1$, under the hypotheses of Theorem 10.1 there are no reasonable topological invariants for the temporal dynamics generated by equation $(0.1)$ on the attractor $\mathcal{A}$. In the one-dimensional case, on the contrary, such invariants do exist. For example, one can take for such an invariant the infimum of $\widehat{h}_{\text {top }}^{1}\left((\mathcal{A}, d), S_{t}\right)$ over all metrics defining the local topology on the attractor by analogy with formula (5.22). Moreover, by (8.16) and the embedding (10.25) this invariant is finite and strictly positive under the hypotheses of Theorem 10.1. Note also that if we attempt to construct an analogous invariant in the multidimensional case, for example, by using the formula

$$
\inf _{d} \widehat{h}_{\mathrm{top}}^{n}\left((\mathcal{A}, d), S_{t}\right)
$$

we clearly obtain the identically zero invariant.

\section{Formally GRADIENT SYSTEMS OF REACTION-DIFFUSION EQUATIONS AND THEIR TOPOLOGICAL ENTROPY}

In this section we consider the special case of equations (0.1) where the nonlinear function $f$ is the gradient of some scalar function $F$ :

$$
f(v)=\nabla_{v} F(v), \quad F \in C^{3}\left(\mathbb{R}^{k}, \mathbb{R}\right) .
$$


Recall that when we consider equation (0.1) in a bounded domain $\Omega$ with zero vector field $\vec{L},(11.1)$ implies that (0.1) has a global Lyapunov function of the following form:

$$
\mathcal{L}(u):=\frac{1}{2} \int_{\Omega}\left(a \nabla_{x} u . \nabla_{x} u+2 F(u)+\lambda_{0} u . u\right) d x .
$$

In particular, since there exists a Lyapunov function, it follows that the topological entropy of the action of the semigroup $S_{t}$ on the attractor is equal to zero:

$$
h_{\text {top }}\left(\mathcal{A}, S_{t}\right)=0 \text {. }
$$

But in the case of the unbounded domain $\Omega=\mathbb{R}^{n}$ the expression (11.2) turns out to be infinite for most $u \in \mathcal{A}$ and so there is no well-defined Lyapunov function on the attractor. Following [27, 37] we say that such systems are formally gradient. Nevertheless, in spite of the lack of a global Lyapunov function, the existence of the gradient structure (11.1) does lead to very substantial simplification of the space-time dynamics generated by equation (0.1). (See, for example, 22, 27, 37 regarding a more detailed study of formally gradient systems in small dimensions $n=1$ and $n=2$.)

The main result of this section is the following theorem, which gives a natural analogue of equality (11.3) for the case of a formally gradient system in $\mathbb{R}^{n}$.

Theorem 11.1. Suppose that the hypotheses of Theorem 1.1 hold and in addition, that (11.1) is satisfied. Then the action of the extended $(n+1)$-parameter semigroup (1.6) on the attractor has zero topological entropy:

$$
h_{\mathrm{top}}\left(\mathcal{A}, \mathbb{S}_{(t, h)}\right)=0 .
$$

Proof. First of all, we note that the vector field $\vec{L}$ involved in equation (0.1) can be reduced to the form $\vec{L}:=(L, 0, \ldots, 0)$ by a suitable orthogonal change of the $x$-coordinate. Moreover, using the following change of $(t, x)$-coordinates, which is standard in the theory of travelling waves,

$$
(t, x)=\mathbb{A}\left(t^{\prime}, x^{\prime}\right), \quad t=t^{\prime}-L x_{1}, \quad x=x^{\prime},
$$

we can reduce the general case of equation (0.1) to the special case $\vec{L}=0$. Clearly, the set $\mathcal{K}$ of all bounded trajectories of $(0.1)$ does not change under this change of coordinates. Moreover, the determinant of this change of coordinates is equal to one; hence by Corollary 3.3 we have

$$
h_{\text {top }}\left(\mathcal{A}, \mathbb{S}_{(t, h)}\right)=h_{\text {top }}\left(\mathcal{K}, \mathbb{T}_{(t, h)}\right)=h_{\text {top }}\left(\mathcal{K}, \mathbb{T}_{\mathbb{A}\left(t^{\prime}, x^{\prime}\right)}\right)=h_{\text {top }}\left(\mathcal{A}^{\prime}, \mathbb{S}_{\left(t^{\prime}, x^{\prime}\right)}\right) .
$$

Here we also use the fact that the mapping $\Pi_{t=0}: \mathcal{K} \rightarrow \mathcal{A}$ is a homeomorphism (see Theorem 5.1 and the proof of Theorem 10.2) and the topological entropy is a topological invariant. Thus, the topological entropy is indeed preserved under transformations of the form (11.5); consequently, it is sufficient to prove Theorem 11.1 only for the case $\vec{L}=0$. For this we consider the space $\mathbb{M}(\mathcal{A})$ of all Borel probability measures on $\mathcal{A}$ which are invariant under the group of spatial translations $\left\{T_{h}, h \in \mathbb{R}^{n}\right\}$. (This space is clearly nonempty, since $\mathcal{A}$ is compact in the local topology of the space $\Phi_{\text {loc }}$.) We define the action of the semigroup $S_{t}^{*}$ generated by equation $(0.1)$ in the space $\mathbb{M}(\mathcal{A})$ in the following standard way:

$$
S_{t}^{*}: \mathbb{M}(\mathcal{A}) \rightarrow \mathbb{M}(\mathcal{A}), \quad\left(S_{t}^{*} \mu\right)(B):=\mu\left(S_{t}^{-1} B\right), \quad B \subset \mathcal{A} .
$$

The next lemma shows that the semigroup (11.6) has a "genuine" Lyapunov function. (This contrasts with the original semigroup $S_{t}$ on the attractor, where there is only a formal Lyapunov function.) 
Lemma 11.1. Suppose that the hypotheses of Theorem 11.1 hold and that $\vec{L}=0$. Then the semigroup (11.6) admits a global Lyapunov function of the following form:

$$
\mathcal{L}(\mu)=\int_{u_{0} \in \mathcal{A}}\left[a \nabla_{x} u_{0}\left(x_{0}\right) \cdot \nabla_{x} u_{0}\left(x_{0}\right)+\lambda_{0} u_{0}\left(x_{0}\right) \cdot u_{0}\left(x_{0}\right)+2 F\left(u_{0}\left(x_{0}\right)\right)\right] \mu(d u) .
$$

In particular, $\mathcal{L}(\mu)$ does not depend on the choice of the point $x_{0} \in \mathbb{R}^{n}$.

Proof. First of all, we observe that the local topologies generated on $\mathcal{A}$ by the embeddings $\mathcal{A} \subset \Phi_{\text {loc }}$ and $\mathcal{A} \subset C_{\text {loc }}^{4-\delta}\left(\mathbb{R}^{n}\right), \delta>0$, coincide. Indeed, by Theorem 5.1 the evolution operator $S_{t}$ realizes a homeomorphism of $\mathcal{A}$ equipped with the topology of the space $\Phi_{\text {loc }}$. On the other hand, by Corollary 1.1 we have

$$
S_{t}:\left(\mathcal{A}, \Phi_{\text {loc }}\right) \rightarrow\left(\mathcal{A}, C_{\text {loc }}^{4-\delta}\left(\mathbb{R}^{n}\right)\right)
$$

for any $\delta>0$ and $t \geq 1$. Hence, in particular, $S_{-t}$ is a continuous operator from $\left(\mathcal{A}, C_{\text {loc }}^{4-\delta}\left(\mathbb{R}^{n}\right)\right)$ into $\left(\mathcal{A}, \Phi_{\text {loc }}\right)$. Moreover, since by Theorem $2.1 \mathcal{A}$ is bounded in $C_{b}^{4-\delta}\left(\mathbb{R}^{n}\right)$ for any $\delta>0, \mathcal{A}$ is compact in $C_{\mathrm{loc}}^{4-\delta}\left(\mathbb{R}^{n}\right)$. Thus, the mapping (11.8) is a homeomorphism for any $\delta>0$ and $t \geq 1$. Representing the identity mapping Id: $\mathcal{A} \rightarrow \mathcal{A}$ in the form of the composite Id $=S_{t} \circ S_{-t}$ we now obtain that Id is a homeomorphism between $\left(\mathcal{A}, \Phi_{\text {loc }}\right)$ and $\left(\mathcal{A}, C_{\text {loc }}^{4-\delta}\left(\mathbb{R}^{n}\right)\right)$. So as required, the above-mentioned topologies do coincide. Hence the functions

$$
u_{0} \rightarrow \partial_{x_{i} x_{j}}^{2} u_{0}\left(x_{0}\right), \quad u_{0} \rightarrow F\left(u_{0}\left(x_{0}\right)\right), \quad u_{0} \rightarrow \partial_{t} \partial_{x_{i}} u_{0}\left(x_{0}\right)
$$

are continuous functions on the attractor $\left(\mathcal{A}, \Phi_{\text {loc }}\right)$. (Henceforth we will use $\partial_{t} u_{0}, u_{0} \in \mathcal{A}$, to mean the result of substituting $u_{0}$ into the left-hand side of equation (0.1).) Since $\mu$ is assumed to be a Borel measure, it follows from the continuity of the function (11.9) that (11.7) is well defined. Moreover, since the measure $\mu \in \mathbb{M}(\mathcal{A})$ is invariant under spatial translations, (11.7) is independent of $x_{0} \in \mathbb{R}^{n}$. Thus, it remains to prove that (11.7) is a Lyapunov function.

To do this, let $\mu$ be an arbitrary measure in $\mathbb{M}(\mathcal{A})$. Then (11.6) and (11.7) give (11.10)

$$
\begin{aligned}
\mathcal{L}(t) & :=\mathcal{L}\left(S_{t}^{*} \mu\right) \\
& =\int_{u_{0} \in \mathcal{A}} 2\left[a \nabla_{x} u\left(t, x_{0}\right) . \nabla_{x} u\left(t, x_{0}\right)+\lambda_{0} u\left(t, x_{0}\right) \cdot u\left(t, x_{0}\right)+2 F\left(u\left(t, x_{0}\right)\right)\right] \mu\left(d u_{0}\right),
\end{aligned}
$$

where $u\left(t, x_{0}\right):=\left(S_{t} u_{0}\right)\left(x_{0}\right)$. By differentiating this expression with respect to $t$ and expressing $\partial_{t} u$ using equation (0.1) we obtain

$$
\begin{aligned}
\frac{d}{d t} \mathcal{L}(t)= & -2 \int_{u_{0} \in \mathcal{A}}\left[\partial_{t} u\left(t, x_{0}\right) \cdot \partial_{t} u\left(t, x_{0}\right)\right] \mu\left(d u_{0}\right) \\
& +2 a \sum_{i=1}^{n} \int_{u_{0} \in \mathcal{A}} \partial_{x_{i}}\left[\partial_{x_{i}} u\left(t, x_{0}\right) . \partial_{t} u\left(t, x_{0}\right)\right] \mu\left(d u_{0}\right) .
\end{aligned}
$$

(We can interchange differentiation with respect to $t$ and integration with respect to the measure $\mu$ because the functions (11.9) are continuous.) We claim that the second summand in (11.11) is equal to zero. Indeed, since the measure $\mu$ is invariant under spatial 
translations, we have

$$
\begin{aligned}
\int_{u_{0} \in \mathcal{A}} & \partial_{x_{i}}\left[\partial_{x_{i}} u\left(t, x_{0}\right) \cdot \partial_{t} u\left(t, x_{0}\right)\right] \mu\left(d u_{0}\right) \\
= & \lim _{h \rightarrow 0} \frac{1}{h} \int_{u_{0} \in \mathcal{A}}\left[\partial_{x_{i}} u\left(t, x_{0}+h e_{i}\right) \cdot \partial_{t} u\left(t, x_{0}+h e_{i}\right)-\partial_{x_{i}} u\left(t, x_{0}\right) \cdot \partial_{t} u\left(t, x_{0}\right)\right] \mu\left(d u_{0}\right) \\
= & \lim _{h \rightarrow 0} \frac{1}{h}\left\{\int_{u_{0} \in \mathcal{A}} \partial_{x_{i}} u\left(t, x_{0}\right) \cdot \partial_{t} u\left(t, x_{0}\right) \mu\left(d u_{0}\right)-\int_{u_{0} \in \mathcal{A}} \partial_{x_{i}} u\left(t, x_{0}\right) \cdot \partial_{t} u\left(t, x_{0}\right) \mu\left(d u_{0}\right)\right\} \\
= & 0 .
\end{aligned}
$$

By integrating (11.11) with respect to $t$ we now obtain

$$
\mathcal{L}\left(S_{t_{2}}^{*} \mu\right)-\mathcal{L}\left(S_{t_{1}}^{*} \mu\right)=-2 \int_{t_{1}}^{t_{2}} \int_{u_{0} \in \mathcal{A}}\left|\partial_{t} u\left(t, x_{0}\right)\right|^{2} \mu\left(d u_{0}\right) \leq 0 .
$$

Thus, (11.7) is nondecreasing along the trajectories of the semigroup (11.6). Now suppose that

$$
\mathcal{L}\left(S_{t_{1}}^{*} \mu\right)=\mathcal{L}\left(S_{t_{2}}^{*} \mu\right)
$$

for some $\mu \in \mathbb{M}(\mathcal{A})$ and $t_{2}>t_{1}$. We claim that the support of $\mu$ must be a subset of the set $\mathcal{R} \subset \mathcal{A}$ of equilibria of (0.1):

$$
\operatorname{supp} \mu \subset \mathcal{R} \text {. }
$$

Indeed, it follows from the spatial invariance of $\mu$ and (11.12) that

$$
\int_{t_{1}}^{t_{2}} \int_{u_{0} \in \mathcal{A}}\left|\partial_{t} u(t, x)\right|^{2} \mu\left(d u_{0}\right) d t=0
$$

for any $x \in \mathbb{R}^{n}$. Thus, for $\mu$-almost all $u_{0} \in \mathcal{A}$ we have

$$
\int_{t_{1}}^{t_{2}}\left|\partial_{t} u(t, x)\right|^{2} d t=0 \quad \forall x \in \mathbb{Q}^{n}
$$

By Corollary 1.3 the function $t \rightarrow \partial_{t} u(t, x)$ is continuous for any $u \in \mathcal{K}$, and so it follows from (11.16) that for $\mu$-almost all $u_{0} \in \mathcal{A}$ we have $\partial_{t} u(t, x) \equiv 0$ for all $t \in\left[t_{1}, t_{2}\right]$ and all $x \in \mathbb{Q}^{n}$. Since the function $x \rightarrow \partial_{t} u(t, x)$ is also continuous, the equality $\partial_{t} u(t, x) \equiv 0$ holds for all $(t, x) \in\left[t_{1}, t_{2}\right] \times \mathbb{R}^{n}$ and therefore $u_{0} \in \mathcal{R}$. Thus, we have proved the equality

$$
\mu(\mathcal{R})=1,
$$

which implies the embedding (11.14). It remains to observe that any measure satisfying (11.14) is an equilibrium of the system (11.6). Lemma 11.1 is proved.

We are now ready to complete the proof of Theorem 11.1. Suppose that $\mu$ is an arbitrary Borel probability measure that is invariant under the action of the extended semigroup (1.6) generated by (0.1). Then, clearly, $\mu$ is an equilibrium of the system (11.6), and (11.14) holds in view of Lemma 11.1. Thus, the metric entropy $h_{\mu}\left(\mathcal{A}, \mathbb{S}_{(t, h)}\right)$ of the action of the semigroup (1.6) on the attractor corresponding to the invariant measure $\mu$ is equal to zero:

$$
h_{\mu}\left(\mathcal{A}, \mathbb{S}_{(t, h)}\right)=0 .
$$

(For a rigorous definition of it, see, for example, [6, 34, 38.) Since (11.18) is valid for any $\mathbb{S}_{(t, h)}$-invariant measure $\mu$, by the variational principle we have

$$
h_{\text {top }}\left(\mathcal{A}, \mathbb{S}_{(t, h)}\right)=\sup _{\mu} h_{\mu}\left(\mathcal{A}, \mathbb{S}_{(t, h)}\right)=0
$$

(see, for example, [34, 38]). Theorem 11.1 is proved. 
Remark 11.1. The existence of a global Lyapunov function for the semigroup generated by a formally gradient system on the set of spatially invariant measures was proved in [37] for small dimensions, $n=1$ and $n=2$. However, in the construction of this Lyapunov function certain specific facts were used, which have no analogues for $n>2$.

Remark 11.2. Note that the topological entropy $h_{\text {top }}\left(\mathcal{A}, S_{t}\right)$ of the one-parameter semigroup $S_{t}$ corresponding to $(0.1)$ is not necessarily equal to zero under the hypotheses of Theorem 11.1. Indeed, consider the following scalar Chafee-Infante equation perturbed by a sufficiently large transport term:

$$
\partial_{t} u=\Delta_{x} u-L \partial_{x_{1}} u+u-u^{3}, \quad x \in \mathbb{R}^{n} .
$$

Then for $L>2$ equation (11.19) obviously satisfies the hypotheses of Theorem 10.2 and therefore

$$
\widehat{h}_{\text {top }}^{n}\left(\mathcal{A}, S_{t}\right) \geq C>0 \quad \text { and } \quad h_{\text {top }}\left(\mathcal{A}, S_{t}\right)=\infty .
$$

On the other hand, condition (11.1) is also satisfied, since equation (11.19) is a scalar one; hence,

$$
h_{\mathrm{top}}\left(\mathcal{A}, \mathbb{S}_{(t, h)}\right)=0
$$

\section{REFERENCES}

[1] A. V. Babin and M. I. Vishik, Attractors of evolution equations, Nauka, Moscow, 1989; English transl., North-Holland, Amsterdam, 1992. MR1156492 (93d:58090)

[2] M. I. Vishik and S. V. Zelik, A regular attractor for a nonlinear elliptic system in a cylindrical domain, Mat. Sb. 190, no. 6 (1999), 23-58; English transl., Sb. Math. 190 (1999), 803-834. MR.1719581 (2000j:35027)

[3] S. V. Zelik, An attractor of a nonlinear system of reaction-diffusion equations in $\mathbb{R}^{n}$ and estimates for its є-entropy, Mat. Zametki 65 (1999), 941-944; English transl., Math. Notes 65 (1999), 790-792. MR.1728296 (2000k:37124)

[4] S. V. Zelik, The attractor of a quasilinear hyperbolic equation with dissipation in $\mathbb{R}^{n}$ : dimension and $\epsilon$-entropy, Mat. Zametki 67 (2000), 304-307; English transl., Math. Notes 67 (2000), 248-251. MR.1768433 (2001c:37099)

[5] O. A. Ladyzhenskaya, V. A. Solonnikov, and N. N. Ural'tseva, Linear and quasi-linear equations of parabolic type, Nauka, Moscow, 1967; English transl., Amer. Math. Soc., Providence, RI, 1968. MR.0241821 (39:3159a)

[6] A. Katok and B. Hasselblatt, Introduction to the modern theory of dynamical systems, Cambridge Univ. Press, Cambridge, 1995. MR1326374 (96c:58055)

[7] A. N. Kolmogorov and V. M. Tikhomirov, $\epsilon$-entropy and $\epsilon$-capacity of sets in functional spaces, Uspekhi Mat. Nauk 14, no. 2 (86) (1959), 3-86; English transl., Amer. Math. Soc. Transl. (2) 17 (1961), 227-364. MR0112032 (22:2890)

[8] A. Milke and S. Zelik, Infinite-dimensional trajectory attractors of elliptic boundary value problems in cylindrical domains, Uspekhi Mat. Nauk 57 no. 4 (346) (2202), 119-150; English transl., Russian Math. Surveys 57 (2002), 753-784. MR1942119(2003i:37083)

[9] M. I. Vishik and V. V. Chepyzhov, Kolmogorov $\epsilon$-entropy of attractors of reaction-diffusion systems, Mat. Sb. 189, no. 2 (1998), 81-110; English transl., Sb. Math. 189 (1998), 235-263 MR1622313 (99c:35115)

[10] F. Abergel, Existence and finite-dimensionality of the global attractor for evolution equations on unbounded domains, J. Differential Equations 83 (1990), 85-108. MR1031379 (90m:58121)

[11] V. Afraimovich, A. Babin, and S.-N. Chow, Spatial chaotic structure of attractors of reactiondiffusion systems, Trans. Amer. Math. Soc. 348 (1996), 5031-5063. MR.1344202 (97c:35087)

[12] V. Afraimovich, A. Babin, and S.-N. Chow, Infinitely spatially complex solutions of PDE and their homotopy complexity, Comm. Anal. Geom. 9 (2001), 281-339. MR1846205 (2002f:35113)

[13] S. Agmon and L. Nirenberg, Lower bounds and uniqueness theorems for solutions of differential equations in a Hilbert space, Comm. Pure Appl. Math. 20 (1967), 207-229. MR0204829 (34:4665)

[14] A. V. Babin and M. I. Vishik, Attractors of partial differential evolution equations in an unbounded domain, Proc. Roy. Soc. Edinburgh, Sect. A 116 (1990), 221-243. MR1084733 (91m:35106)

[15] R. Boas, Entire functions, Academic Press, New York, 1954. MR0068627 (16:914f)

[16] L. A. Bunimovich and Ya. G. Sină̌, Spacetime chaos in coupled map lattices, Nonlinearity 1 (1988), 491-516. MR0967468 (90d:58073) 
[17] À. Calsina, X. Mora, and J. Solà-Morales, The dynamical approach to elliptic problems in cylindrical domains, and a study of their parabolic singular limit, J. Differential Equations 102 (1993), 244-304. MR.1216730 (94e:35094)

[18] V. V. Chepyzhov and M. I. Vishik, Attractors for equations of mathematical physics, Amer. Math. Soc., Providence, RI, 2002. MR1868930 (2003f:37001c)

[19] P. Collet and J.-P. Eckmann, Extensive properties of the complex Ginzburg-Landau equation, Comm. Math. Phys. 200 (1999), 699-722. MR.1675141 (2000b:35234)

[20] P. Collet and J.-P. Eckmann, The definition and measurement of the topological entropy per unit volume in parabolic PDEs, Nonlinearity 12 (1999), 451-473. MR1690187 (2000m:37180)

[21] P. Collet and J.-P. Eckmann, Topological entropy and $\varepsilon$-entropy for damped hyperbolic equations, Ann. Inst. Henri Poincaré 1 (2000), 715-752. MR1785186 (2001m:35203)

[22] J.-P. Eckmann and J. Rougemont, Coarsening by Ginzburg-Landau dynamics, Comm. Math. Phys. 199 (1998), 441-470. MR.1666859 (2000e:35206)

[23] M. A. Efendiev and S. V. Zelik, The attractor for a nonlinear reaction-diffusion system in an unbounded domain, Comm. Pure Appl. Math. 54 (2001), 625-688. MR,1815444 (2001m:35035)

[24] M. A. Efendiev and S. V. Zelik, Upper and lower bounds for the Kolmogorov entropy of the attractor for the RDE in an unbounded domain, J. Dynam. Differential Equations 14 (2002), 369-403. MR1901023 (2003d:35021)

[25] E. Feireisl, Bounded, locally compact global attractors for semilinear damped wave equations on $\mathbf{R}^{N}$, Differential Integral Equations 9 (1996), 1147-1156. MR1392099 (97f:35138)

[26] B. Fiedler and C. Rocha, Orbit equivalence of global attractors of semilinear parabolic differential equations, Trans. Amer. Math. Soc. 352 (2000), 257-284. MR1475682 (2000c:35129)

[27] Th. Gallay and S. Slijepčević, Energy flow in formally gradient partial differential equations on unbounded domains, J. Dynam. Differential Equations 13 (2001), 757-789. MR1860285 (2002h:35195)

[28] J. Hale, Asymptotic behavior of dissipative systems, Amer. Math. Soc., Providence, RI, 1987. MR0941371 (89g:58059)

[29] K. Kirchgässner, Wave-solutions of reversible systems and applications, J. Differential Equations 45 (1982), 113-127. MR0662490 (83j:35063)

[30] O. Ladyzhenskaya, Attractors for semigroups and evolution equations, Cambridge Univ. Press, Cambridge, 1991. MR1133627 (92k:58040)

[31] E. Lindenstrauss and B. Weiss, Mean topological dimension, Israel J. Math. 115 (2000), 1-24. MR.1749670 (2000m:37018)

[32] A. Mielke and G. Schneider, Attractors for modulation equations on unbounded domains-existence and comparison, Nonlinearity 8 (1995), 743-768. MR1355041 (97e:58207)

[33] A. Mielke and S. Zelik, Attractors of reaction-diffusion systems in $\mathbb{R}^{n}$ with strictly positive spatiotemporal topological entropy, in preparation.

[34] J. Moulin-Ollagnier and D. Pinchon, The variational principle, Studia Math. 72 (1982), 151-159. MR.0665415 (83j:28019)

[35] Ya. B. Pesin and Ya. G. Sinaı̆, Space-time chaos in chains of weakly interacting hyperbolic mappings, Adv. Soviet Math., vol. 3, Dynamical systems and statistical mechanics (Moscow, 1991), Amer. Math. Soc., Providence, RI, 1991, pp. 165-198. MR.1118162 (93a:58108)

[36] D. Ruelle, Turbulence, strange attractors, and chaos, World Scientific Publishing, River Edge, NJ, 1995. MR1407035 (97f:58091)

[37] S. Slijepčević, Extended gradient systems: dimension one, Discrete Contin. Dynam. Systems 6 (2000), 503-518. MR1757384 (2001i:37133)

[38] A. T. Tagi-Zade, A variational characterization of the topological entropy of continuous groups of transformations. The case of $\mathbb{R}^{n}$-actions, Mat. Zametki 49, no. 3 (1991), 114-123; English transl., Math. Notes 49 (1991), 305-311. MR:1110315 (92b:58132)

[39] R. Temam, Infinite-dimensional dynamical systems in mechanics and physics, Springer-Verlag, New York, 1988. MR0953967|(89m:58056)

[40] H. Triebel, Interpolation theory, function spaces, differential operators, North-Holland, Amsterdam, 1978. MR0503903 (80i:46032b)

[41] S. V. Zelik, The attractor for a nonlinear reaction-diffusion system in the unbounded domain and Kolmogorov's $\epsilon$-entropy, Math. Nachr. 232 (2001), 129-179. MR.1871475 (2002k:35043)

[42] S. V. Zelik, The attractor for a nonlinear hyperbolic equation in the unbounded domain, Discrete Contin. Dynam. Systems 7 (2001), 593-641. MR1815770 (2001m:35230) 
[43] S. V. Zelik, Attractors of reaction-diffusion systems in unbounded domains and their spatial complexity, Comm. Pure Appl. Math. 56 (2003), 584-637. MR1953652 (2004a:37113)

[44] S. Zelik, Spatial and dynamical chaos generated by reaction diffusion systems in unbounded domains, DANSE, FU-Berlin, Preprint 38/00, 2000, pp. 1-60.

University of Stuttgart, Germany

E-mail address: zelik@mathematik.uni-stuttgart.de

Translated by E. KHUKHRO 